A RECONFIGURABLE COMPUTING PLATFORM FOR REAL TIME EMBEDDED APPLICATIONS

A THESIS SUBMITTED TO

THE GRADUATE SCHOOL OF NATURAL AND APPLIED SCIENCES

$\mathrm{OF}$

MIDDLE EAST TECHNICAL UNIVERSITY

BY

FATIH SAY

IN PARTIAL FULFILLMENT OF THE REQUIREMENTS

FOR

THE DEGREE OF PHILOSOPHY OF DOCTORATE

IN

ELECTRICAL AND ELECTRONICS ENGINEERING

SEPTEMBER 2011 
Approval of the thesis:

\section{A RECONFIGURABLE COMPUTING PLATFORM FOR REAL TIME EMBEDDED APPLICATIONS}

submitted by FATIH SAY in partial fulfillment of the requirements for the degree of Philosophy of Doctorate in Electrical and Electronics Engineering Department, Middle East Technical University by,

Prof. Dr. Canan Özgen

Dean, Graduate School of Natural and Applied Sciences

Prof. Dr. İsmet Erkmen

Head of Department, Electrical and Electronics Engineering

Assoc. Prof. Dr. Cüneyt F. Bazlamaçcı

Supervisor, Electrical and Electronics Engineering Dept., METU

\section{Examining Committee Members:}

Prof. Dr. Hasan Cengiz Güran

Electrical and Electronics Engineering Dept., METU

Assoc. Prof. Dr. Cüneyt Fehmi Bazlamaçcı

Electrical and Electronics Engineering Dept., METU

Prof. Dr. Volkan Atalay

Computer Engineering Dept., METU

Prof. Dr. Semih Bilgen

Electrical and Electronics Engineering Dept., METU

Assist. Prof. Dr. Oğuz Ergin

Computer Engineering Dept., TOBB ETÜ

Date: 
I hereby declare that all information in this document has been obtained and presented in accordance with academic rules and ethical conduct. I also declare that, as required by these rules and conduct, $I$ have fully cited and referenced all material and results that are not original to this work.

Name, Last Name: FATIH SAY

Signature 


\begin{abstract}
A RECONFIGURABLE COMPUTING PLATFORM FOR REAL TIME EMBEDDED APPLICATIONS
\end{abstract}

\author{
SAY, Fatih \\ Ph.D., Department of Electrical and Electronics Engineering \\ Supervisor : Assoc. Prof. Dr. Cüneyt F. Bazlamaçcı
}

September 2011, 136 pages

Today's reconfigurable devices successfully combine 'reconfigurable computing machine' paradigm and 'high degree of parallelism' and hence reconfigurable computing emerged as a promising alternative for computing-intensive applications. Despite its superior performance and lower power consumption compared to general purpose computing using microprocessors, reconfigurable computing comes with a cost of design complexity. This thesis aims to reduce this complexity by providing a flexible and user friendly development environment to application programmers in the form of a complete reconfigurable computing platform.

The proposed computing platform is specially designed for real time embedded applications and supports true multitasking by using available run time partially reconfigurable architectures. For this computing platform, we propose a novel hardware task model aiming to minimize logic resource requirement and the overhead due to the reconfiguration of the device. Based on this task model an optimal 2D surface partitioning strategy for managing the hardware resource is presented. A mesh network-on-chip is designed to be used as the communication environment for the hardware tasks and a runtime mapping technique is employed to lower the communication overhead. 
As the requirements of embedded systems are known prior to field operation, an offline design flow is proposed for generating the associated bit-stream for the hardware tasks. Finally, an online real time operating system scheduler is given to complete the necessary building blocks of a reconfigurable computing platform suitable for real time computing-intensive embedded applications.

In addition to providing a flexible development environment, the proposed computing platform is shown to have better device utilization and reconfiguration time overhead compared to existing studies.

Keywords: Reconfigurable Computing, Hardware Real Time Operating System, Hardware Partitioning, Network-on-chip 


\title{
$\ddot{0} \mathrm{z}$
}

\section{GERÇEK ZAMANLI UYGULAMALAR İÇIN YENIDEN YAPILANDIRILABILININIR BILIŞIIM PLATFORMU}

\author{
SAY, Fatih \\ Doktora, Elektrik-Elektronik Mühendisliğ Bölümü \\ Tez Yöneticisi : Doç. Dr. Cüneyt Fehmi Bazlamaçcı
}

Eylül 2011,136 sayfa

Günümüzün yeniden yapılandırılabilinir donanımları, 'yeniden yapılandırılabilinir bilişim’ paradigmasını ve 'yüksek dereceli paralelliği' birlikte sunmaktadır. Bu donanımlarla yeniden yapılandırılabilinir bilişim, yoğun işlem gücü gerektiren uygulamalar için gelecek vaat eden bir alternatif çözüm olarak ortaya çıkmıştır. Mikroişlemcilerin kullanıldı̆̆ 1 genel amaçlı bilişime kıyasla üstün performans ve düşük güç tüketimi sunmasına karşın, yeniden yapılandırılabilinir bilişim oldukça karmaşık bir tasarım süreci gerektirmektedir. Bu tez çalışması yeniden yapılandırılabilinir bilişim platformunu bir bütün halinde sunarak ve uygulama geliştirenler için esnek ve kullanıcı dostu bir geliştirme ortamı sağlayarak bu karmaşıklığı azaltmayı amaçlamaktadır.

Önerilen bilişim platformu gerçek zamanlı gömülü uygulamalar için özel olarak tasarlanmış olup, çalışma sırasında donanımın kısmen yeniden yapılandırılmasını destekleyen donanım mimarileri kullanarak gerçek anlamda çoklu görevleri desteklemektedir. Bu bilişim ortamında kullanılmak üzere, uygulamalar için ihtiyaç duyulan donanım kaynak gereksinimi ve bu donanımın yeniden yapılandırmasının getirdiği ek zaman yükünü asgariye indirmek amacı ile yeni bir donanım görev modeli öneriyoruz. Bu görev modeline dayalı olarak, do- 
nanım kaynaklarını en uygun biçimde yöneten iki boyutlu bir donanım yüzeyi bölümleme stratejisi sunulmaktadır. Bu donanım yüzeyinde donanımsal görevler için haberleşme ortamını sağlamak için gözenek yapıda bir yonga üstü ağ tasarlanmıştır. Ayrıca bu ağ üstündeki iletişim yükünü azaltmak için çalışma anında donanım üstüne uygun yerleşimi yapacak teknik geliştirilmiştir.

Gömülü sistemlerin ihtiyaçları önceden bilindiğinden, donanım görevlerinin yapılandırma bilgilerinin çevrim dışı bir tasarım akışı ile oluşturulması önerilmektedir. Son olarak, gerçek zamanlı ve yüksek işlem gücü gerektiren gömülü uygulamalara uygun bir yeniden yapılandırılabilinir bilişim ortamının gerekli tüm bileşenlerini sağlamak için çevrimiçi ve gerçek zamanlı işletim sistemi görev zamanlayıcısı tanımlanmıştır.

Esnek bir geliştirme ortamı sağlamanın yanında, önerilen bilişim ortamının literatürdeki çalışmalara göre daha iyi donanım kaynak kullanımı ve daha kısa yeniden yapılandırma süresi sunduğu gösterilmiştir.

Anahtar Kelimeler: Yeniden Yapılandırılabilinir Bilişim, Donanımsal Gerçek Zamanıı İşletim Sistemi, Donanım Bölüştürmesi, Yonga Üstü A $\breve{g}$ 
To my wife, my son and my little daughter 


\section{ACKNOWLEDGMENTS}

First, I must thank my advisor, Assoc. Prof. Dr. Cüneyt Fehmi Bazlamaçcı for his support during both my MSc and PhD studies. This work would not have been possible without his guidance, advice, criticism and encouragements.

I would also like to express my gratitude to my dissertation committee members; Prof. Dr. Hasan Cengiz Güran and Prof. Dr. Volkan Atalay. They have provided many useful insights and helpful feedback during this process.

I would like to acknowledge my company ASELSAN Inc. for the encouragement and support during my MSc and PhD studies.

Last but not the least, I must thank my wife, Şifa Say, whose love and support are crucial for anything I have ever accomplished. I also thank our kids, Eren and Berra for bringing me sunshine and happiness. 


\section{TABLE OF CONTENTS}

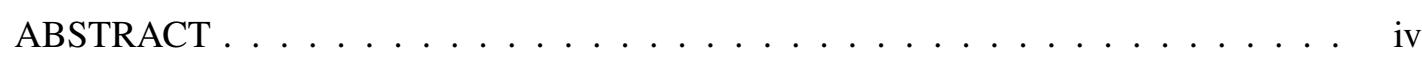

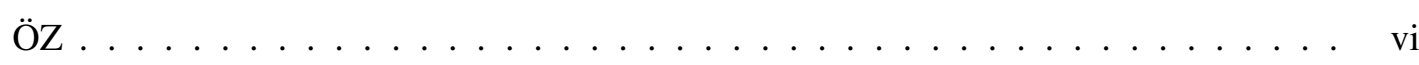

ACKNOWLEDGMENTS . . . . . . . . . . . . . . . . . . ix

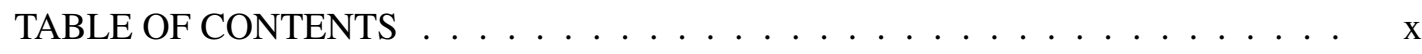

LIST OF TABLES $\ldots \ldots \ldots \ldots \ldots \ldots \ldots \ldots \ldots \ldots$ xiii

LIST OF FIGURES . . . . . . . . . . . . . . . . . . . . . . . . . xiv

LIST OF ABBREVIATIONS $\ldots \ldots \ldots \ldots \ldots \ldots$. . . . . . . . . . . . . . . . . . . . . . .

\section{CHAPTERS}

$1 \quad$ INTRODUCTION $\ldots \ldots \ldots \ldots \ldots \ldots \ldots$

$1.1 \quad$ Motivation . . . . . . . . . . . . . . . 3

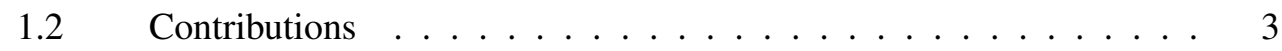

1.3 Thesis Organization $\ldots \ldots \ldots \ldots \ldots$

$2 \quad$ BACKGROUND AND RELATED WORK $\ldots \ldots \ldots \ldots$

$2.1 \quad$ Surface Partitioning and Placement . . . . . . . . . . 6

$2.2 \quad$ Context Loading . . . . . . . . . . . . . . . . . . 12

$2.3 \quad$ Operating System Support $\ldots \ldots \ldots$. . . . . . . . . . . 13

3 COMPUTING PLATFORM MODEL $\ldots \ldots \ldots \ldots \ldots$

$3.1 \quad$ Operating System Model $\ldots \ldots \ldots$

$3.2 \quad$ Reconfigurable Hardware Model $\ldots \ldots \ldots$

$3.3 \quad$ Hardware Task Model $\ldots \ldots \ldots \ldots$

$3.4 \quad$ Execution Block Model $\ldots \ldots \ldots \ldots$

$3.5 \quad$ Surface Partitioning Model $\ldots \ldots \ldots$

$3.6 \quad$ Placement Model $\ldots \ldots \ldots \ldots$. . . . . . . . . . . . . . 32 
$3.7 \quad$ Reduced Hardware Task Model . . . . . . . . . . . . . . . . . . . 32

$3.8 \quad$ Context Loading Model $\ldots \ldots \ldots$. . . . . . . . . . . . . 34

$4 \quad$ USER BLOCK SIZE SELECTION $\ldots \ldots \ldots \ldots$

$4.1 \quad$ Problem Formulation $\ldots \ldots \ldots \ldots$

$4.2 \quad$ Problem Analysis $\ldots \ldots \ldots \ldots$

$4.3 \quad$ Literature survey on BPP-1 $\ldots \ldots \ldots \ldots$. . . . . . . . 41

$4.4 \quad$ Greedy Heuristic for User Block Size Selection $\ldots \ldots \ldots$

$4.5 \quad$ Quality Analysis for Greedy Heuristic . . . . . . . . . . . . . . 44

5 INTER BLOCK COMMUNICATION NETWORK ARCHITECTURE . . . 46

$5.1 \quad$ Communication Requirements $\ldots \ldots \ldots$. . . . . . . . . 46

$5.1 .1 \quad$ Task Level Communication Requirements . . . . . . . . 47

$5.1 .2 \quad$ Memory Access Requirements . . . . . . . . . . . 47

5.1.3 Operating System Services Communication Requirements 48

$5.2 \quad$ Literature Survey on $\mathrm{NoC} \ldots \ldots \ldots$

$5.3 \quad$ Inter Block Communication Network Architecture . . . . . . . . . . 51

$5.3 .1 \quad$ IBCN Topology $\ldots \ldots \ldots \ldots$. . . . . . . . . 52

$5.3 .2 \quad$ IBCN Switching and Flow Control . . . . . . . . . . 53

$5.3 .3 \quad$ IBCN Routing $\ldots \ldots \ldots \ldots \ldots$

$5.3 .4 \quad$ IBCN Switch Structure $\ldots \ldots \ldots \ldots$

$5.3 .5 \quad$ IBCN Packet Format $\ldots \ldots \ldots \ldots \ldots$

6 HARDWARE TASK PLACEMENT AND MAPPING PROBLEM . . . . . . 62

$6.1 \quad$ Literature Survey on Mapping Problem . . . . . . . . . . . . . . . 63

$6.2 \quad$ Traffic Modeling . . . . . . . . . . . . . . . . . . . . 65

6.3 Problem Definition . . . . . . . . . . . . . . . . . . . . 67

6.4 Problem Formulation $\ldots \ldots \ldots \ldots \ldots$

$6.5 \quad$ Problem Analysis $\ldots \ldots \ldots \ldots \ldots$. . . . . . . . . . 71

$6.6 \quad$ Ad-Hoc Mapping Solution $\ldots \ldots \ldots \ldots$. . . . . . . . . 72

$6.6 .1 \quad$ Phase-1: Region Allocation $\ldots \ldots \ldots$. . . . . 73

6.6.2 Phase-2: User Block Placement _. . . . . . . . . . . 77

$6.7 \quad$ Quality Analysis for The Mapping $\ldots \ldots \ldots \ldots$ 
7 HARDWARE OPERATING SYSTEM $\ldots \ldots \ldots \ldots$. . . . . . . . . 81

$7.1 \quad$ Offline Bitstream Generation . . . . . . . . . . . . . . . . 82

$7.2 \quad$ Hardware Operating System Components . . . . . . . . . . . . . . 83

7.2.1 Hardware Operating System Kernel _. . . . . . . . . . 84

7.2.2 Host Communication Controller . . . . . . . . . . . . 85

$7.2 .3 \quad$ Local Communication Controller. . . . . . . . . . . . 85

7.2.4 Hardware Task Scheduler. . . . . . . . . . . . . . . 86

$7.2 .5 \quad$ Mapping Engine $\ldots \ldots \ldots \ldots$. . . . . . . . . 89

$7.2 .6 \quad$ Reconfiguration Manager. . . . . . . . . . . . . . . . 90

8 IMPLEMENTATION OF THE RECONFIGURABLE COMPUTING PLAT-

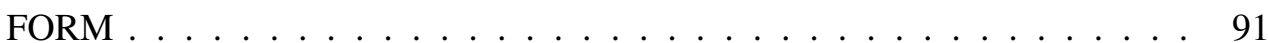

$8.1 \quad$ IBCN Implementation Results . . . . . . . . . . . . . . . . . . . . 91

$8.2 \quad$ Mapping Engine Implementation Results $\ldots \ldots \ldots$. . . . . . . . . . 94

$8.2 .1 \quad$ Free Rectangle List Generator Circuitry . . . . . . . . . . 94

$8.2 .2 \quad$ Phase-1 Circuitry . . . . . . . . . . . . 96

$8.2 .3 \quad$ Phase-2 Circuitry . . . . . . . . . . . . . . . . . 98

$8.3 \quad$ System Area Circuitry Implementation Results . . . . . . . . . . . . 100

9 COMPUTATIONAL STUDY $\ldots \ldots \ldots \ldots$. . . . . . . . . . . . . 102

9.1 Computational Study Results on Reconfigurable Device Utilization . 102

$9.2 \quad$ Computational Study Results on Reconfiguration Overhead . . . . . 105

9.3 Comparison Results on Device Utilization and Reconfiguration Over-

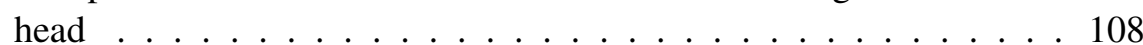

10 APPLICATION EXAMPLE $\ldots \ldots \ldots \ldots$. . . . . . . . . . . . . . 110

11 CONCLUSIONS AND FUTURE WORK $\ldots \ldots \ldots \ldots$

$11.1 \quad$ Contributions . . . . . . . . . . . . . . . . . 115

$11.2 \quad$ Future Work . . . . . . . . . . . . . . . . . . . . . . 117

REFERENCES ... . . . . . . . . . . . . . . . . . . . . . . 119

APPENDIX

A $\quad$ COMPUTATIONAL STUDY RESULTS . . . . . . . . . . . . . . . . . . . . 125

CURRICULUM VITAE $\ldots \ldots \ldots \ldots \ldots \ldots \ldots$ 


\section{LIST OF TABLES}

\section{TABLES}

Table 5.1 IBCN message types and their characteristics . . . . . . . . . . . . . . . . 49

Table 5.2 IBCN flit type encoding . . . . . . . . . . . . . . . . . . . . 61

Table 8.1 IBCN FIFO implementation $\ldots \ldots \ldots$. . . . . . . . . . . . . . . . 92

Table 8.2 IBCN virtual channel FIFO implementation . . . . . . . . . . . . . . . . . 93

Table 8.3 IBCN switch control logic implementations . . . . . . . . . . . . . . . 93

Table 8.4 IBCN switch implementations . . . . . . . . . . . . . . . . . . 93

Table 8.5 Circuit size and performance of SAC components on Xilinx Virtex6 LX760 100

Table 10.1 Parameters of individual hardware tasks in the application example . . . . . 113 


\section{LIST OF FIGURES}

\section{FIGURES}

Figure 1.1 Performance and cost comparison of ASIC, GPP and programmable hardware 2

Figure $2.1 \quad$ Arbitrary shaped hardware task circuitry based surface partitioning $\ldots . .8$

Figure $2.2 \quad$ Rectangle shaped hardware task circuitry based surface partitioning . . . . 8

Figure 2.3 An example 1D block partitioning of the reconfigurable hardware surface . 10

Figure $2.4 \quad$ An example 2D block partitioning of the reconfigurable hardware surface . 12

Figure 3.1 Computational model overview $\ldots \ldots \ldots \ldots$

Figure 3.2 Operating system model $\ldots \ldots \ldots$. . . . . . . . . . . . 20

Figure $3.3 \quad$ CLB architecture for Altera Stratix-4 f . . . . . . . . . . . . . . . 21

Figure 3.4 CLB architecture for Xilinx Virtex-4 . . . . . . . . . . . . . . . . . . . 21

Figure 3.5 Universal reconfigurable hardware assumption $\ldots \ldots \ldots \ldots$. . . . . . . . 22

Figure 3.6 Spatial partitioning on the independent data set $\ldots \ldots \ldots$. . . . . . . 25

Figure 3.7 Spatial partitioning on the dependent data set . . . . . . . . . . . . 25

Figure 3.8 An example hardware task dependency graph $\ldots \ldots \ldots \ldots$. . . . . . . . . . . 27

Figure 3.9 An interface template for an execution block . . . . . . . . . . . . . . . . 29

Figure $3.103 \times 3$ user block allocation . . . . . . . . . . . . . . . . . . . 30

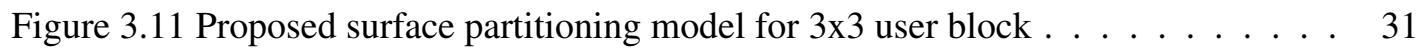

Figure 3.12 Merging example: Four reconfigurable devices merged into a virtual reconfigurable device . . . . . . . . . . . . . . . 31

Figure 3.13 A placement example: surface partitioning for a set of four tasks . . . . . . 32

Figure 3.14 a) An example hardware task b) its reduced hardware task . . . . . . . . 33

Figure 4.1 Problem of user block size selection $\ldots \ldots \ldots$. . . . . . . . . . . . 37 
Figure 4.2 Pseudo code for greedy heuristic user block size selection $\ldots$. . . . . . . . . 43

Figure 4.3 Pseudo code for finding effective circuit size $\ldots \ldots \ldots$. . . . . . . . . 44

Figure 5.1 IBCN topology for $3 \times 3$ surface partitioning $\ldots \ldots \ldots \ldots$. . . . . . . . . 53

Figure 5.2 IBCN switch structure $\ldots \ldots \ldots \ldots$. . . . . . . . . . . . . . 56

Figure 5.3 Physical location of IBCN switch buffers $\ldots \ldots \ldots \ldots$

Figure 5.4 Two virtual channel based buffer architecture in IBCN $\ldots \ldots \ldots$. . . . . 57

Figure 5.5 An example $5 \times 5$ IBCN topology with FIFOs $\ldots \ldots \ldots$. . . . . . . . . 58

Figure 5.6 IBCN Switch control logic architecture . . . . . . . . . . . . . . . . . . . 59

Figure 5.7 A switching table for a switch having virtual channels in horizontal direction 60

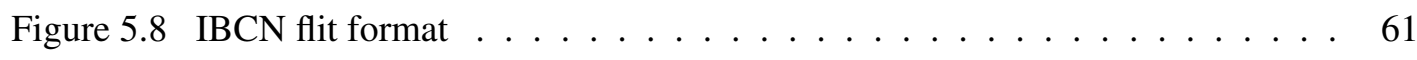

Figure $6.1 \quad$ An example traffic demand for user blocks of a reduced task graph level . . 67

Figure 6.2 An example hardware surface usage and memory traffic load for access

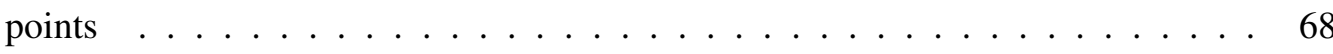

Figure 6.3 Physical mapping of the user blocks onto reconfigurable hardware surface to minimize the Manhattan distance $\ldots \ldots \ldots$. . . . . . . . . . . . . . 69

Figure 6.4 Physical mapping of the user blocks onto reconfigurable hardware surface to balance access point loads $\ldots \ldots \ldots$. . . . . . . . . . . . . 69

Figure 6.5 Physical mapping of the user blocks onto reconfigurable hardware surface to minimize latency due to path blocking . . . . . . . . . . . . . . . 70

Figure $6.6 \quad$ A non rectangle shaped mapping disturbing existing communication. . . . . 74

Figure 6.7 Pseudo code for region allocation phase . . . . . . . . . . . . . . . . 76

Figure $6.8 \quad$ A virtual rectangle based mapping example $\ldots \ldots \ldots$. . . . . . . . . . . . 79

Figure 7.1 Offline bitstream generation process $\ldots \ldots \ldots \ldots$. . . . . . . . . . . 83

Figure 7.2 Hardware operating system components . . . . . . . . . . . . . . . . . . 84

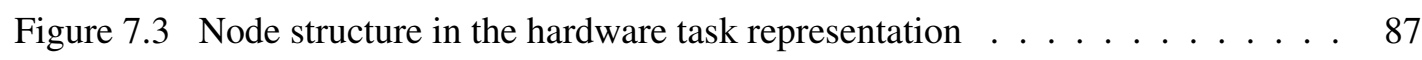

Figure 8.1 Free rectangle list generator block diagram . . . . . . . . . . . . . . . 95

Figure 8.2 Worst case condition for free list generation circuitry $\ldots \ldots$. . . . . . . 96 
Figure 8.3 Region allocation circuitry block diagram $\ldots \ldots$. . . . . . . . . . . . 97

Figure 8.4 Physical mapping circuitry block diagram $\ldots \ldots \ldots$. . . . . . . . . . . 98

Figure 8.5 SAC implementation for Xilinx Virtex6 LX760 . . . . . . . . . . . . . . . 101

Figure 9.1 Ratio of effective_size() to whole circuit size with respect to $P F$ for $T S R=1.75$ case $\ldots \ldots \ldots \ldots$. . . . . . . . . . . . . . 104

Figure $9.2 \rho_{\text {total }}$ with respect to $P F$ for $T S R=1.75$ case $\ldots \ldots \ldots \ldots$

Figure 9.3 Ratio of $\rho_{\text {total }}$ with our proposal to that of 2D fixed partitioning [20,21] against $P F$ for $T S R=1 \ldots \ldots \ldots \ldots \ldots \ldots$

Figure 9.4 Ratio of $\rho_{\text {total }}$ with our proposal to that of 2D fixed partitioning [20,21] against $T S R$ for $E B S R=1 \ldots \ldots$. . . . . . . . . . . . 106

Figure 9.5 Reconfiguration overhead as percentage of total task turn around time variation against ET2RTR for different values of $P F \ldots \ldots \ldots$

Figure 9.6 Achieved reconfiguration overhead as percentage of reconfiguration overhead without task partitioning versus ET2RTR for different values of $P F \quad \ldots$

Figure 9.7 Ratio of reconfiguration overhead our proposal to reconfiguration overhead with $[26,27]$ against $P F \quad \ldots \ldots \ldots$

Figure 9.8 Ratio of $\rho_{\text {total }}$ with our proposal to that of $[26,27]$ against $P F \quad \ldots \ldots$

Figure 10.1 Application example: Image and sensor fusion to detect and track airborne threats . . . . . . . . . . . . . . . . . . . . . . 110

Figure 10.2 Complete reconfigurable computing solution for the application example . 112

Figure A.1 Ratio of effective_size() to whole circuit size with respect to $P F$ for

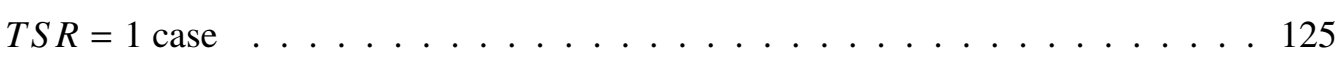

Figure A.2 $\rho_{\text {total }}$ with respect to $P F$ for $T S R=1$ case $\ldots \ldots \ldots$. . . . . . 126

Figure A.3 Ratio of effective_size() to whole circuit size with respect to $P F$ for

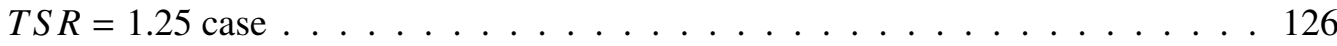

Figure A.4 $\rho_{\text {total }}$ with respect to $P F$ for $T S R=1.25$ case $\ldots \ldots \ldots$. . . . . . . . 127

Figure A.5 Ratio of $\rho_{\text {total }}$ with our proposal to that of 2D fixed partitioning [20,21] against $P F$ for $T S R=1.25 \ldots \ldots$. . . . . . . . . . . . . . . . . 127 
Figure A.6 Ratio of effective_size() to whole circuit size with respect to $P F$ for

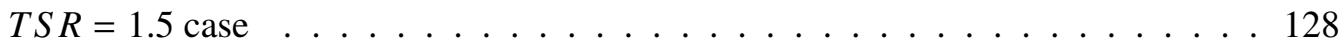

Figure A.7 $\rho_{\text {total }}$ with respect to $P F$ for $T S R=1.5$ case $\ldots \ldots \ldots \ldots$

Figure A.8 Ratio of $\rho_{\text {total }}$ with our proposal to that of 2D fixed partitioning [20,21] against $P F$ for $T S R=1.5 \ldots \ldots \ldots$

Figure A.9 Ratio of $\rho_{\text {total }}$ with our proposal to that of 2D fixed partitioning [20,21] against $P F$ for $T S R=1.75 \ldots \ldots$. . . . . . . . . . . . . . . . . . . . . . . . 129

Figure A.10Ratio of effective_size() to whole circuit size with respect to $P F$ for

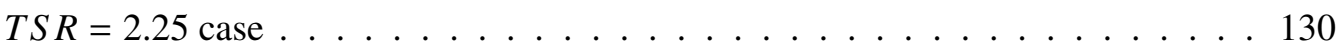

Figure A.11 $\rho_{\text {total }}$ with respect to $P F$ for $T S R=2.25$ case . . . . . . . . . . . . . . . 130

Figure A.12Ratio of $\rho_{\text {total }}$ with our proposal to that of 2D fixed partitioning [20,21]

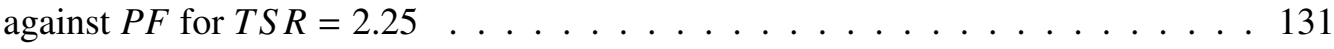

Figure A.13Ratio of effective_size() to whole circuit size with respect to $P F$ for $T S R=3$ case $\ldots \ldots \ldots \ldots \ldots \ldots \ldots$

Figure A.14 $\rho_{\text {total }}$ with respect to $P F$ for $T S R=3$ case $\ldots \ldots \ldots 132$

Figure A.15Ratio of $\rho_{\text {total }}$ with our proposal to that of 2D fixed partitioning [20,21] against $P F$ for $T S R=3 \ldots \ldots \ldots \ldots$. . . . . . . . . . . . . . 132

Figure A.16Ratio of effective_size() to whole circuit size with respect to $P F$ for

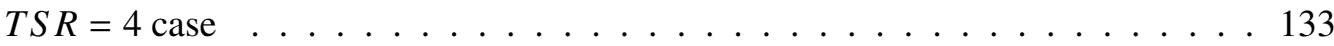

Figure A.17 $\rho_{\text {total }}$ with respect to $P F$ for $T S R=4$ case $\ldots \ldots \ldots 133$

Figure A.18Ratio of $\rho_{\text {total }}$ with our proposal to that of 2D fixed partitioning [20,21] against $P F$ for $T S R=4 \ldots \ldots$. . . . . . . . . . . . . . . . . . . . . . . . . . 134

Figure A.19Ratio of effective_size() to whole circuit size with respect to $P F$ for $T S R=5$ case $\ldots \ldots \ldots \ldots \ldots$. . . . . . . . . . . . . . . . . . . . .

Figure A.20 $\rho_{\text {total }}$ with respect to $P F$ for $T S R=5$ case $\ldots \ldots \ldots \ldots$

Figure A.21Ratio of $\rho_{\text {total }}$ with our proposal to that of 2D fixed partitioning [20,21] against $P F$ for $T S R=5 \ldots \ldots \ldots$. . . . . . . . . . . . . . 135 


\section{LIST OF ABBREVIATIONS}

$\begin{array}{ll}\text { AP } & \text { : Access Point } \\ \text { BPP-1 } & : \text { One dimensional Bin Packing Problem } \\ \text { CLB } & : \text { Configurable Logic Block } \\ \text { EB } & : \text { Execution Block } \\ \text { ET2RTR } & : \text { Execution Time to Reconfiguration Time Ratio } \\ \text { GPP } & : \text { General Purpose Processor } \\ \text { IBCN } & : \text { Inter Block Communication Network } \\ \text { PF } & : \text { Parallelism Factor } \\ \text { PRTR } & : \text { Partially Run-Time Reconfigurable } \\ \text { SAC } & : \text { System Area Circuitry } \\ \text { UB } & : \text { User Block } \\ \text { UBS } & : \text { User Block Size }\end{array}$




\section{CHAPTER 1}

\section{INTRODUCTION}

The classical computer with general purpose processors (GPP) is based on Von-Neumann Model, where the application program resides in memory and at each cycle, an instruction is fetched, decoded and executed. GPP based computation has lots of advantages, like design flexibility, architecture compatibility, high level application programming and operating system support. However, due to this strong serial execution cycle, execution time is bounded by cycle time, which is limited. Another limitation is due to underlying processor instruction set. The same generic instruction set must be used for different applications. Therefore we need an alternative solution for the computing intensive applications.

In order to get rid of the limitation of Von-Neumann computing mode, the reconfigurable computing machine paradigm is introduced as a potential remedy for computing-intensive applications by Estrin [1]. This paradigm is based on a fixed plus variable architecture, where the fixed part is similar to GPP and variable part is a hardware whose behavior changes during the program execution. The power of this paradigm is the variable hardware which can perform a computation in a parallel and optimum manner. As a first case study, Jacobean matrix multiplication application is mapped to this computing model and 4 to 10 times execution speed improvement is achieved [1].

The idea of reconfigurable computing was not practical, until the invention of first reconfigurable device; Xilinx SRAM based Field Programmable Gate Array (FPGA), in the 80s. Until this milestone, the alternative for GPP is to use an application specific integrated circuit (ASIC), which achieves the best performance in terms of both execution time and power consumption. However, ASIC comes with a large non-recurring engineering cost and time to market and with no design flexibility to adopt to changes in the system. In the early days, 
the FPGAs were used just as a fast prototype and low cost replacement to ASICs. As depicted in Figure-1, the FPGAs provide an intermediate solution in terms of design cost and performance between GPP and ASIC for the design of embedded systems.

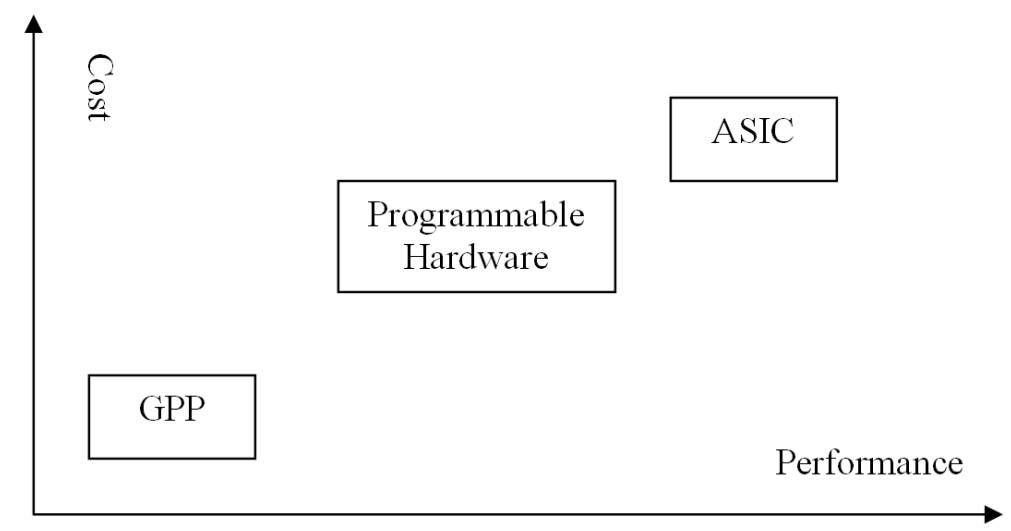

Figure 1.1: Performance and cost comparison of ASIC, GPP and programmable hardware

Being a fine grained architecture, the first generations of FPGA were requiring too much configuration time to load context for configuration and it was only allowed to reconfigure the whole FPGA. Therefore, these devices were not suitable for reconfigurable computing platforms requiring the hardware to be changed frequently. Hence, some coarse grained reconfigurable hardware architectures (like, MorphoSys [2] and Chimera [3]) are proposed. These devices are configured in very short time. However, as these devices have very limited logic resources, their application area is very limited and cannot be used in computing intensive applications. On the other hand, with large number of logical resources and massive parallelism features, the first generations of FPGA were useful as a co-processor to accelerate part of an application and 10 to 100 times performance was achieved in terms of execution time. The literature includes a number of case studies that demonstrate such performance gain [2-7]. Also, FPGAs are used in commercial available high performance computing (HPC) clusters such as Cray X1 [8] and SGI RASC RC200 [9].

The idea of reconfigurable computing paradigm became more realistic and powerful with the introduction of partially run-time reconfigurable (PRTR) FPGA, which allows reprogramming one part of the reconfigurable hardware in a short time while the remaining part is still operational. With this feature, the PRTR FPGA became a candidate for the variable hard- 
ware. Besides, partially run-time reconfiguration PRTR allows context loading of more than one application onto device and thus allows true multitasking.

\subsection{Motivation}

The reconfigurable devices like FPGAs were designed to provide the performance levels of ASIC with the design flexibility similar to software development. For these purposes, high level hardware description languages like Verilog and VHDL are developed. These languages with commercially available synthesis and placement routing tools allow the application programmer to develop his circuitry without burdening himself into the underlying hardware details. However, this flexibility is valid if the reconfigurable devices are used as an alternative to ASIC.

Despite its advantages in execution time improvements and true multiprocessing, the reconfigurable computing doesn't give the design flexibility as compared to software development for GPP. For the GPP based computing platform, there are lots of operating systems that manage the computing resources and hide the details of underlying hardware from the user. In addition, an application developed for a GPP based computing platform can run on a different computing GPP based computing platform without any difficulty.

To the best of our knowledge, existing reconfigurable computing platforms and the resource management techniques are far away from the flexibility provided by GPP. Therefore, in this thesis study a complete reconfigurable computing platform with necessary design support and management technique is presented. While hiding details of the reconfigurable computing platform, our management technique utilizes the hardware resources in an efficient manner to boost system performance.

\subsection{Contributions}

The contributions of this dissertation can be summarized as follows:

- A novel hardware task model for a reconfigurable computing platform that targets a true multitasking environment for real time and computing-intensive embedded applications 
is presented. This task model results in better reconfigurable hardware utilization and faster task turnaround time as compared to existing methods.

- The surface of reconfigurable hardware is managed using the proposed task model. For this purpose, a novel 2D surface partitioning strategy is proposed using a mathematical model for finding the optimal surface partitioning parameters. The resulting optimization problem is then solved using a greedy heuristic based on one-dimensional bin packing problem.

- A 2D mesh network-on-chip (NoC) is specially developed as the communication environment for handling the requirements of the proposal.

- An ad-hoc online mapping technique is presented to keep the communication overhead on the proposed NoC low.

- An offline bit stream generation process is formulated and presented.

- Finally, based on the developed surface partitioning strategy and the task model, a real time hardware operating system scheduler is proposed to support true hardware multitasking.

The proposed components form all the necessary building blocks of a complete reconfigurable computing platform suitable for real time computing-intensive embedded applications.

Results of our extensive computational study show that a considerable performance improvement is achieved compared to existing methods for different type of applications classified by circuit size and parallelism. In addition, a real life sensor fusion application example suitable for our proposal is presented. This sensor fusion application which detects and tracks threats in an airborne defense system also indicates how well our proposal is.

\subsection{Thesis Organization}

The rest of the thesis is organized as follows. First, an extensive literature survey on existing resource management and operating system approaches for reconfigurable computing is given in Chapter 2. Chapter 3 presents the assumed reconfigurable hardware model and our novel hardware task model including its corresponding execution block model (hardware 
template). Our 2D surface partitioning approach is also presented in Chapter 3. Starting from Chapter 4, we give the implementation details of the components of the proposed reconfigurable computing platform. Our mathematical formulation, analysis and solution of optimal 2D surface partitioning process are presented in Chapter 4. Then the architectural details of the 2D mesh network-on-chip (NoC) with a literature survey on existing NoC architectures is given in Chapter 5. Chapter 6 introduces our mapping problem. After a brief related work subsection, the details of our ad-hoc mapping technique are discussed. In Chapter 7, our special hardware operating system scheduler supporting real time requirements and off-line bit-stream generation process are all discussed. In Chapter 8, an implementation of our reconfigurable computing platform is given based on a commercially available FPGA and the logic requirements of our management circuitry and $\mathrm{NoC}$ architecture is given. The performance of our proposal is evaluated on an extensive computational study in Chapter 9. Chapter 10 includes a real life application example suitable to be implemented on our proposed platform. Finally this thesis ends with our conclusions. 


\section{CHAPTER 2}

\section{BACKGROUND AND RELATED WORK}

With today's sophisticated commercial tools, an application programmer can develop applications using commercially available reconfigurable hardware. This development process is straightforward as long as all the tasks can fit into the targeting reconfigurable hardware. However, when the overall logic requirements of the tasks exceed the hardware logic capacity, then special techniques are needed to manage the reconfigurable hardware and schedule the tasks by configuring its hardware context onto the reconfigurable hardware over time. From the application programmer's perspective, the development of this mapping and scheduling techniques is not an easy job. Therefore a reconfigurable computing platform with necessary management and scheduling support is needed.

This thesis work aims to provide a complete reconfigurable computing platform for computing intensive real time embedded applications. The computing platform objectives are to give a design flexibility to an application programmer and manage the reconfigurable hardware in such a way that both device utilization is maximized and execution time of the tasks are minimized.

While achieving these objectives, the proposed solution leverages the existing studies in literature. In the upcoming section, a discussion of the distinction of this study from those existing works is given.

\subsection{Surface Partitioning and Placement}

In our reconfigurable computing platform, true multitasking is supported and hence the related context of the circuitry for the tasks must be loaded onto the reconfigurable device surface at 
runtime. Therefore there is a need for a management technique to partition the reconfigurable device surface.

The performance criterion for the surface partitioning technique is the utilization of available reconfigurable hardware surface. This reconfigurable hardware utilization factor directly affects system performance. For non real time systems, if the reconfigurable hardware utilization is low, the tasks must wait for a long time to have enough free space and start context loading, which will result in a slower computing platform in the sense of execution time. In a similar fashion, for real time systems the effect of low reconfigurable hardware utilization will be large task rejection ratio if it is allowed to reject task when there is not enough free space. In this case, it is the system designer's responsibility to handle such rejected tasks, which makes the computing platform much more complicated to users. If task rejection is not allowed, then a larger reconfigurable hardware is needed and this will result in larger cost and power consumption, which are very critical factors in today's embedded systems.

Surface partitioning techniques also determine the circuitry shape of the hardware tasks. An attempt to support arbitrary shaped hardware task circuitry was made by Deng et al. [10]. As depicted in Figure 2.1, the reconfigurable hardware surface is partitioned into regions with size and shape of the hardware task circuitry to be scheduled. Generally, the commercial synthesis, placement and routing tools generate arbitrary shaped hardware task circuitry to minimize the propagation delay and achieve maximum operating frequency for the task. Despite these advantages, this arbitrary shaped hardware task circuitry based surface partitioning technique suffers from fragmentation and have very low device utilization.

In order to solve the low reconfigurable hardware utilization problem with arbitrary shaped hardware task circuitry, the footprint transformation technique was proposed by Walder and Platzner [11]. The proposed technique first tries to find a free space with the size and shape of the hardware task circuitry to be scheduled. In case of failure, if there is enough free contiguous space, a footprint transformation technique is applied to fit the circuitry to available free space. The authors reported that almost $25 \%$ of the hardware tasks were footprint transformed during scheduling of a set of hardware tasks in a computational study to achieve better reconfigurable hardware utilization. However, as this footprint transformation requires placement and routing process, which are time consuming, this proposed technique is not suitable in real time applications. 


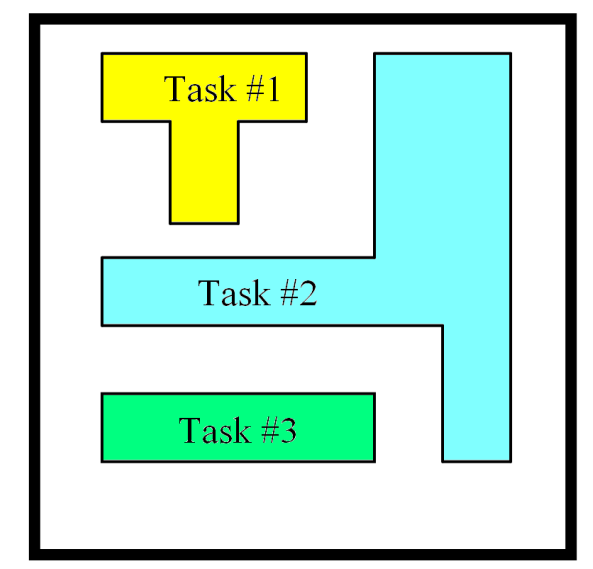

Figure 2.1: Arbitrary shaped hardware task circuitry based surface partitioning

In an effort to achieve lower fragmentation and hence better reconfigurable hardware utilization, the hardware task circuitry are restricted to be in rectangular shape in a number of studies [12-14]. In this partitioning methodology, the reconfigurable hardware surface is partitioned into rectangles equal to the size of hardware task circuitry (see Figure 2.2). Bazargan et al. [12] proposed both offline and online placement and surface partitioning technique for rectangle shaped hardware task circuitry. The offline placement algorithm was specially designed for reconfigurable architectures not supporting run time partially reconfigurability. The online placement algorithm was a two step generic algorithm. In the first step, the empty space is partitioned into a set of disjoint 'empty rectangles'. Among the suitable size empty rectangles the $2 \mathrm{D}$ bin packing rules are applied to favor one over the others.

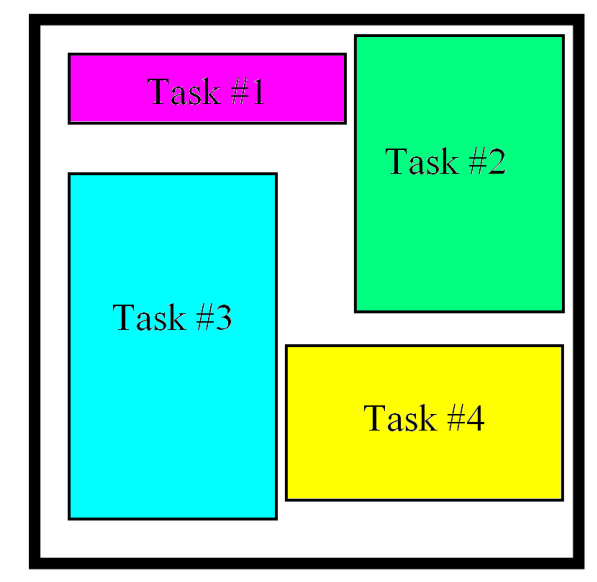

Figure 2.2: Rectangle shaped hardware task circuitry based surface partitioning 
Walder et al. [13] presented an improvement to the rectangle shaped online task placement given in [12]. A hash matrix data structure is used to represent the free rectangles on the reconfigurable hardware surface and it takes constant time to find the best free rectangle instead of $\mathrm{O}(\mathrm{n})$ time complexity of [12]. Indeed, Walder et al. [13] reported an approximately $70 \%$ utilization improvement as compared to [12].

Instead of keeping a list of free rectangles that are disjoint to each other, Gu et al. [14] introduced an efficient algorithm to find a complete set of maximal empty rectangles and gave a hardware implementation of this algorithm. This new algorithm was an improvement to [15], where an empty list of maximal empty rectangles are found in an efficient manner. In addition to this fast rectangle search algorithm, an online placement technique with feature aware behavior was presented to minimize the reconfigurable hardware surface fragmentation and hence minimize task rejection ratio.

Recently, in 2010, an online placement technique was introduced by Hu et al. [79] for hardware task with rectangle shape. With an efficient data structure representing the FPGA surface usage, the candidate locations for the scheduled hardware task is found and an adjacency based heuristic technique is used to find the most suitable one among these candidate locations. Whenever a hardware task is placed or completes its execution, the FPGA surface usage information is updated with an algorithm having $O\left(n^{2}\right)$ time complexity.

No matter how efficient the partitioning and placement technique is, it is still a high probability to have fragmentation on the reconfigurable hardware surface if online task scheduling is needed. A remedy for this fragmentation problem was proposed by Diessel et al. [16] by rearranging a subset of the placed tasks to allow next pending task to be processed sooner. Being an NP-hard problem, a genetic algorithm is applied for rearrangement in bounded time. However, this technique is not applicable to real time systems due to time required for rearrangement.

From the above discussions, it is obvious that the rectangle shaped hardware task circuitry based surface partitioning and placement suffers from fragmentation for online scheduling in real time systems. Another limitation is that there is no communication media for hardware task circuitry to external memory and any possible host processor. Moreover, the above partitioning techniques do not have any communication path between hardware tasks. As our computing platform aims to provide the design flexibility of software development with GPP 
and support the operating system services and inter-task communication, we need a communication media between tasks, a possible external host and memory. Therefore, the rectangle based partitioning techniques [10,11,12,13,14,15 and 79] are not applicable for our computing platform.

In order to provide the communication path to external memory and any host processor with inter-task communication support, block partitioning of the reconfigurable hardware surface is generally applied. An example one dimensional (1D) block partitioned reconfigurable hardware surface is shown in Figure 2.3, where part of the device is used for operating system, part of the device for communication as a shared bus and the rest for the user applications.

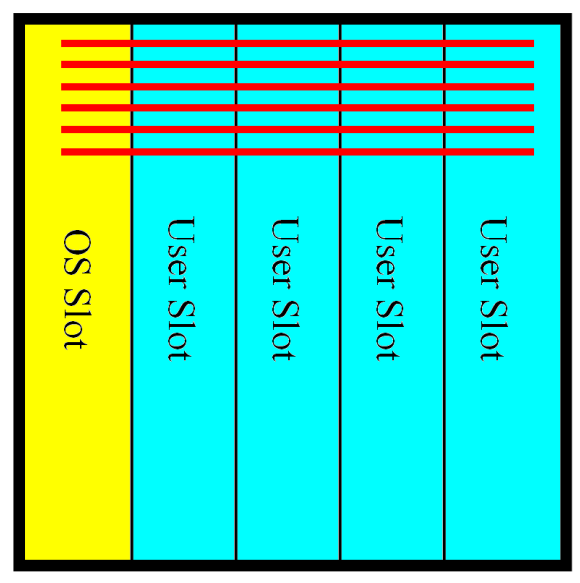

Figure 2.3: An example 1D block partitioning of the reconfigurable hardware surface

A block partitioning approach was introduced by Brebner and Diessel [17], where reconfigurable hardware surface is first partitioned into three vertical parts (top one for operating system, middle one for communication bus and lower one for user tasks). The user task area was partitioned into fixed size 1D tiles and hardware tasks circuitry context was loaded onto required number of tiles. In order to have better device utilization, ordered compaction was applied whenever execution of a hardware task was completed, i.e. all executing tasks are shifted left till there is no fragmented area. However, this compaction requires stopping, relocating and restarting of hardware tasks, which is not an acceptable time overhead in real time systems.

With the aim of supporting real time systems, a 1D surface partitioning and placement technique was developed by Walder and Platzner [18]. Similar to Figure 2.3, the top side and left 
side of the reconfigurable hardware surface was allocated for communication media and operating system respectively. The remaining area was partitioned into fixed depth but variable width 1D tiles. The idea behind such a variable sized block was to minimize the internal fragmentation, i.e., fragmentation within a tile, within the 1D tile itself to achieve better reconfigurable device utilization. Whenever a hardware task was scheduled, the best fit technique was used to select the tile. Because of online requirements and unknown arrival times it is difficult to select the 1D tile width and this technique will suffer from fragmentation also. In another study, the authors proposed partitioning user area into fixed size blocks called 'dummy task' [19]. Whenever a hardware task was scheduled a number of contagious dummy task are used for scheduling. Similar to [17] this technique also suffers from external fragmentation and requires compaction.

In addition to fragmentation problems, the 1D surface partitioning approaches have limitations in communication media and hardware task circuitry operating frequency. Due to shared bus architecture, the communication media bandwidth must be shared between all communicating pairs. Indeed the overall bandwidth is limited due to long propagation delay on the bus. Similarly the operating frequency of the hardware task circuitry is limited due to long wiring delay in the 1D tile, which has large depth to width ratio.

In order to overcome the limitations of $1 \mathrm{D}$ reconfigurable hardware surface partitioning technique, two dimensional (2D) reconfigurable hardware surface partitioning methodology was proposed by Marescaux et al. [20]. As depicted in Figure 2.4, fixed size 2D blocks are allocated for hardware task circuitry context and the remaining area is allocated for system area. The system area was used to implement a switching network on chip with folded torus topology as communication media and operating system related implementations. A very similar surface partitioning technique was applied in [21] also.

With the 2D reconfigurable hardware surface partitioning technique, the communication requirements of the computing platform can be met in an efficient manner. In addition, with 2D block shape, it is possible to have better placement and routing resulting in faster operating clock frequency as compared to 1D tile based partitioning. However, since the 2D block size is fixed and a hardware task circuitry must fit onto a single block, the block size must be chosen as equal to the largest hardware task circuitry at least. As a result, the partitioning presented in [20-21] suffers from internal fragmentation. 


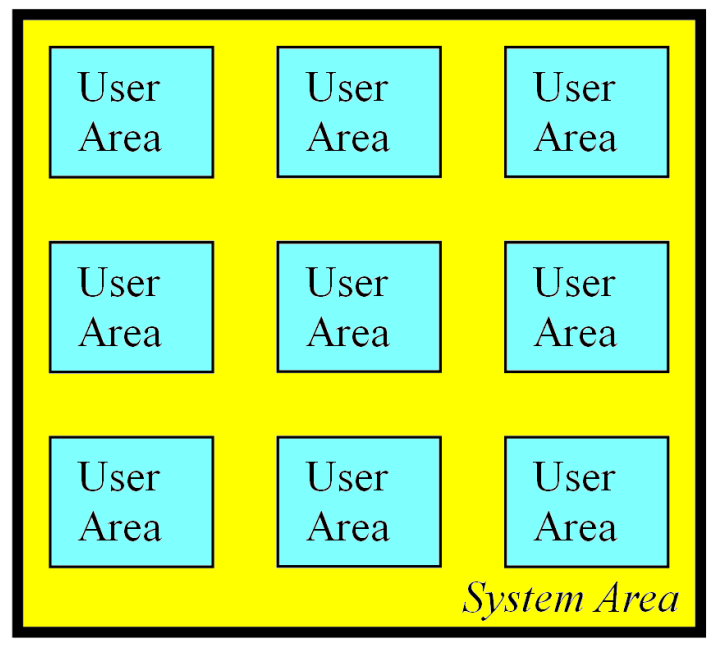

Figure 2.4: An example 2D block partitioning of the reconfigurable hardware surface

\subsection{Context Loading}

The major drawback of today's reconfigurable devices is the time needed to load the context of a hardware task circuitry, i.e. the time needed for reconfiguring the associated bitstream. This results in a considerable timing overhead on the reconfigurable devices. It has been reported that up to $98 \%$ of total time was spent for reconfiguration in run-time reconfigurable computing applications [22]. A similar result was presented by Wirlhlin and Hutchings [23] and it has been shown that performance improvement with reconfigurable computing is limited to 23 times instead of 80 for a specific application due to this context loading time penalty.

As an alternative coarse grained reconfigurable hardware architectures that allow faster context loading compared to fine grained FPGA architectures with large bitstream size [2,3] were presented. However, due to limited reconfigurable resources, these devices are lack of providing massive parallelism.

In order to minimize this time overhead, the multi-context FPGA idea was introduced in [24]. In this model, up to four different bitstream can be stored on the FPGA and the FPGA context can be switched from one configuration to another in just one clock cycle. But this fast response comes with the constraint of reconfiguring whole device and this is not suitable for multiprocessing.

Although reconfiguration interfaces are becoming faster with the advances in silicon technol- 
ogy, reconfiguration time will still be a limiting factor for performance improvement in reconfigurable computing because the whole bit-stream must be read from the external memory and then written to the reconfigurable hardware configuration interface. Since reconfiguration time is directly related to circuit size, Bauer et al. [25] minimized this timing overhead by starting the execution just after loading a part of the circuitry in a reconfigurable processor design. This part of the circuit must be temporarily partitionable from the rest of the circuitry. While this part of circuit is operating the context loading of the rest takes place.

A very similar idea was used by Resano et al. [26] to minimize the reconfiguration overhead in a periodic application with 2D surface partitioned FPGA reconfigurable computing platform. In this architecture, the hardware task circuitry was required to be partitioned into fixed sized sub-tasks and these sub-tasks are required to be temporarily independent. By applying a pipelined context loading and execution scheme, the reconfiguration overhead is kept at a low level. The authors extended their work in [27] and applied the reuse of previously loaded subtasks to further minimize the reconfiguration overhead in periodic applications like MPEG decoders. Although this technique is very efficient in reducing the reconfiguration time overhead, it requires the user to partition the task into fixed size temporal parts. This requirement cannot be met in general and the size of these partitions can vary, which will result in internal fragmentation and hence low device utilization.

\subsection{Operating System Support}

In a user friendly reconfigurable computing platform a hardware operating system is needed to manage the reconfigurable hardware, to schedule the context loading of the hardware tasks and to give high level services to application programmer such as inter-task communication, system calls and semaphores.

In the field of reconfigurable computing, the idea of an operating system was first discussed for Xilinx XC6200, which was the first run time partially reconfigurable hardware [28]. In this reconfigurable computing platform the FPGA area was partitioned into fixed size hardware building blocks called swappable logic units (SLU) that can perform specific functions by using its input and generate results to its output. SLUs can be configured as a bus accessible sea of accelerators and neighboring SLUs can be configured as specific circuits. However this 
approach lacks design flexibility and does not provide support for high level services.

A complete operating system package, named as ReconfigMe, which manages loading of hardware tasks on FPGA and arranges them on FPGA surface, was introduced by Wigley et al. [29]. In ReconfigMe operating system, the hardware tasks were composed of Java like classes and a task graph was used to represent data dependency. The main steps of scheduling were i) allocation process, where free space was compared to hardware task size, ii) placement process, where the hardware task was first pre-packed into allocated rectangular shape and iii) partitioning, where the task was partitioned to reduce the number of classes in case of placement failure. The partitioning phase was followed by allocation and a placement phase in an iterative manner till parts of the partitioned task was placed. Otherwise the task was queued back to the ready queue. Although high level application development support was given, the ReconfigMe doesn't provide any communication media and requires time for partitioning phase, which is not acceptable in real time systems.

With the aim of maximizing the whole resource utilization and reducing the reconfiguration overhead for a hybrid computing platform with a GPP and reconfigurable hardware, an online partitioning algorithm was proposed for real time operating system services in [30]. The proposal assumes that user tasks are available in both binary and bit-stream format and 0-1 integer programming was used online to determine whether the task should run on the GPP or on the reconfigurable hardware. However, this work lacks the information on how to manage the reconfigurable hardware resources, high level services and requires a considerable time for 0-1 integer programming.

For a similar hybrid system with an ARM processor and Xilinx 6264 FPGA, an FPGA support system (FSS) was studied by Edwards and Green in [31] to support placement, execution and removal of a hardware task on the FPGA with communication support to software tasks. The FSS was used for high level support to user, e.g. for hardware task creation, task deletion, task to task communication, task suspend and resume operations. Despite of such user friendly features, the management of the reconfigurable hardware was still within the user's responsibility, where the user must manually load the context on the FPGA.

In [32], a scheduling technique was discussed to support a set of periodic real time hardware tasks for an embedded system with an offline guarantee for the feasibility of the task set. A hardware task was specified by its period, worst case execution time and reconfigurable 
hardware resource requirements. Hardware tasks were merged into a set of servers in such a way that a server will reserve execution time and reconfigurable hardware area for its task set. The proposed operating system kernel schedules a server for a while and pre-empts it and schedules another one. However, this scheduling requires full reconfiguration capability of the device and is not suitable for multitasking environment.

With the aim of lowering power consumption and using smaller FPGAs in automotive domain, an on demand run time system was proposed in [33], where FPGA was partitioned into a number of fixed sized 1D modules communicating over a shared bus module. A soft microprocessor core was also implemented to manage reconfiguration and external communication with sensors. The run time system loads functional modules onto FPGA modules depending on their timing criteria, priority, communication rate and input buffer requirements. However due to limitations of 1D partitioning and shared bus, this technique was not suitable for our demands.

A UNIX like operating system, called BORPH, was introduced for hybrid computing by Kwork and Brodersan [34]. This operating system supports a single hardware task for each FPGA in the computer system and the hardware task executes as a normal UNIX process and the OS supports all high level operating system features including file system and Internet access. The BORPH operating system was implemented on a reconfigurable computer with four FPGAs for user tasks and a central FPGA with an internal CPU for management and general purpose processing. Although very sophisticated high level operating system services were supported, the task size is limited to the quarter of the FPGA and for a small sized task this will result in poor reconfigurable hardware utilization.

The above discussion on related work indicates that the major operating system responsibilities for reconfigurable computing are;

1. Management of the reconfigurable hardware area

2. Performing task scheduling

3. Providing inter-task communication between both hardware and software tasks in the computing system for high level services.

As summarized in [35], performance metrics for operating system are the reconfigurable hard- 
ware utilization, task rejection ratio for hard real time systems, task scheduling turnaround time for soft real time systems, hardware or software resource requirements for operating system itself, the execution time of the applications and the quality of high level services provided from the application programmer's point of view. 


\section{CHAPTER 3}

\section{COMPUTING PLATFORM MODEL}

We can categorize today's computing platforms as;

- Personal Computing

- Enterprise Computing

- Embedded Computing

- High Performance Computing.

The GPP based computers are very suitable for personal computing and small-middle sized enterprise computing areas. Due to large computing power requirements, enterprise computing can be a candidate area for reconfigurable computing and a number of reconfigurable computers with FPGA based clusters to form a supercomputer is already commercially available [8,9]. Since supercomputers are used in well defined scientific and large sized enterprise applications, the main goal is to boost the performance. Therefore, design flexibility, power consumption and unit cost become a second order figure of merits.

On the other hand, embedded applications require high computing power, design flexibility, low power consumption and low unit cost at the same time. Since this figure of merits can be met using reconfigurable computing paradigm, we select embedded applications as our target computing area.

For the computing platform that we are proposing, the reconfigurable hardware is the heart of system, which allows boosting the performance in computing-intensive tasks with its massive parallelism and true multitasking support. However, not all of the tasks in embedded systems 
are suitable to be implemented in hardware and hence we still need a GPP computing resource. In this thesis study, we assume a reconfigurable computational model composed of basically a GPP, a reconfigurable hardware and a unified memory as shown in Figure-3.1.

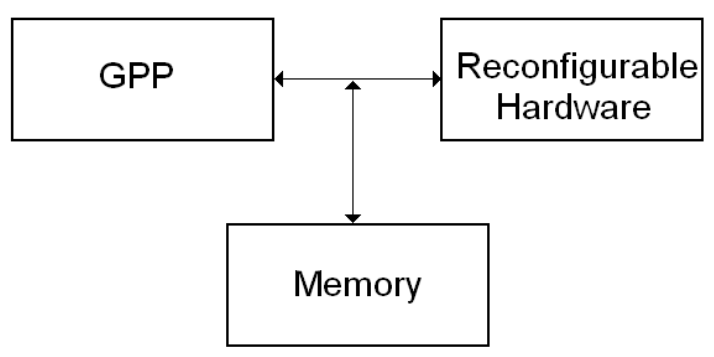

Figure 3.1: Computational model overview

It is possible to design such a reconfigurable computer with just a single commercially available FPGA, which supports internal memory and a soft microprocessor such as Microblaze in Xilinx FPGAs [36] or Nios in Altera FPGAs [37]. Another alternative is to use a hard coded high performance microprocessor in FPGAs, such as PowerPC 440 core in Virtex-5FX FPGAs [38]. For higher performance it is of course possible to use a powerful GPP and a large size reconfigurable hardware. As the GPP type and the coupling between GPP and reconfigurable hardware is system dependent, these issues are not addressed in this study.

The unified memory architecture is used to have a single memory for both GPP and reconfigurable hardware access. This memory is used as data and program memory for GPP. For the reconfigurable hardware, this memory is used as a data memory and to store the bitstream of application part implemented in hardware.

In the following sections, we provide the assumptions about our computing model and present the overall structure of the building blocks of the reconfigurable computing platform we built for real time embedded applications. This platform should have an operating system and reconfigurable hardware. The hardware tasks, composed of execution blocks, should be placed and executed on the reconfigurable hardware surface through a sequence of context loading operations. 


\subsection{Operating System Model}

The proposed reconfigurable computing platform is designed to support both computation with GPP and reconfigurable hardware. In this study, we define the computation using GPP resources as 'software task'. The one using the reconfigurable hardware resources are defined as 'hardware task'. The decision of whether a task should be a hardware task or software task is known as hardware/software co-design, which is an intense research topic in literature. In our reconfigurable computing platform the hardware/software co-design is the user's responsibility and beyond the scope of this study.

In conventional computing platforms, operating systems are used to hide the details of underlying hardware from the application programmer and to give support for high level services, such as inter-task communication, file system and network. In addition, the embedded systems come with real time requirements and this requirement is handled by the operating system also. For the same purposes, we have an operating system in our reconfigurable computing platform too. The operating system is composed of two parts; a 'software operating system' and a 'hardware operating system'.

The software operating system part can easily be selected among the commercial real time operating systems, such as VxWorks [39] or RT Linux [40]. The hardware operating system, which is special to our reconfigurable computing platform, has two parts. The first part is a piece of software running on the GPP as part of the software operating system kernel. The second part is a hardware circuitry and is implemented on the reconfigurable hardware. The responsibility of the first part is to provide support to high level operating system services for hardware tasks and provide a seamless connectivity between hardware tasks and software tasks. The hardware part is responsible for the management of the reconfigurable hardware.

The above discussion is summarized and the relationship between GPP, reconfigurable hardware, software task, hardware task and operating system components are depicted in Figure 3.2 . 


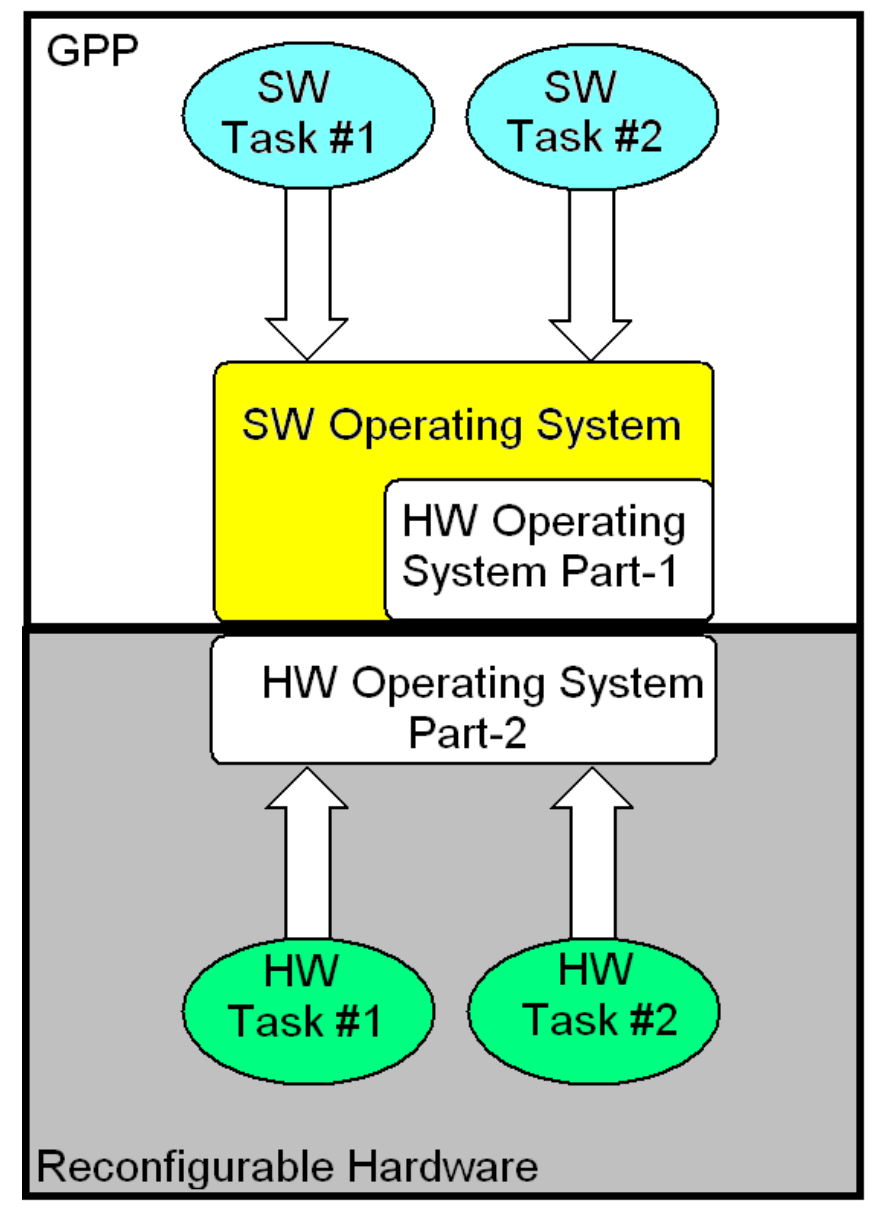

Figure 3.2: Operating system model

\subsection{Reconfigurable Hardware Model}

The reconfigurable hardware assumption throughout this study is a universal device with; logic blocks, connections boxes and interconnect switches, similar to SRAM based commercial FPGA architecture.

The logic block is assumed as a collection of logic gates and storage units. Commercial FPGAs has such logic blocks called 'configurable logic block (CLB)'. The CLB resources for Altera Stratix-4 [41] and Xilinx Virtex-4 [42] architectures are shown in Figure 3.3 and Figure 3.4 respectively. Coarse grained CLBs provide rich set of logic gates but require more reconfiguration time compared to fine grained CLBs. In our reconfigurable computing platform the granularity selection is a user responsibility. 


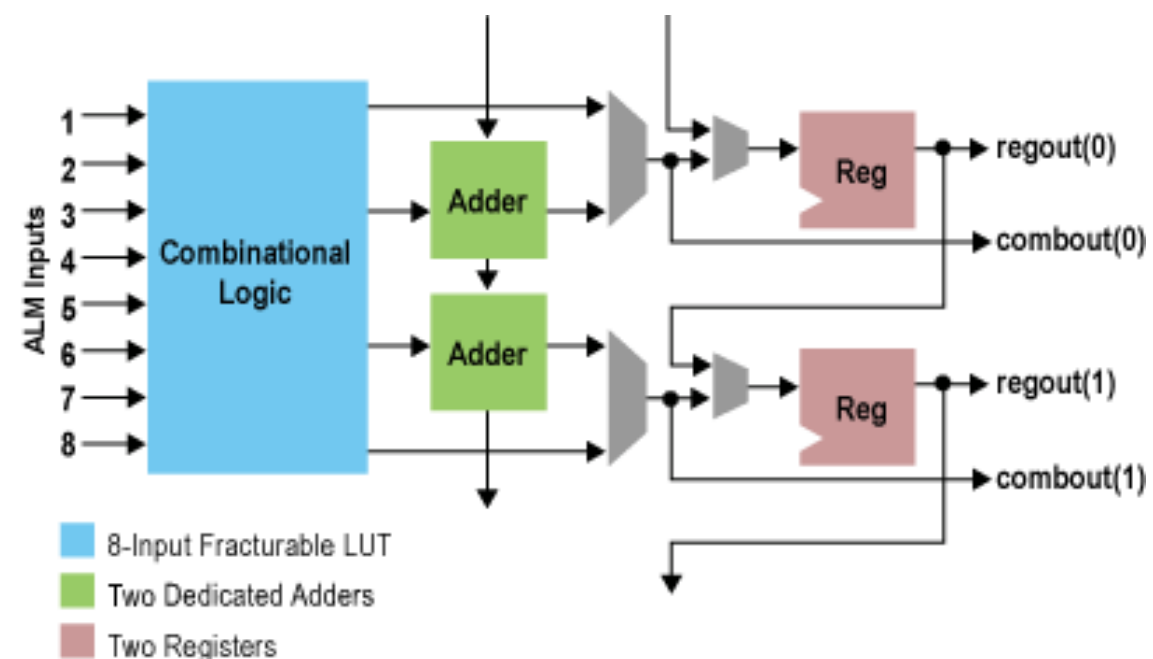

Figure 3.3: CLB architecture for Altera Stratix-4

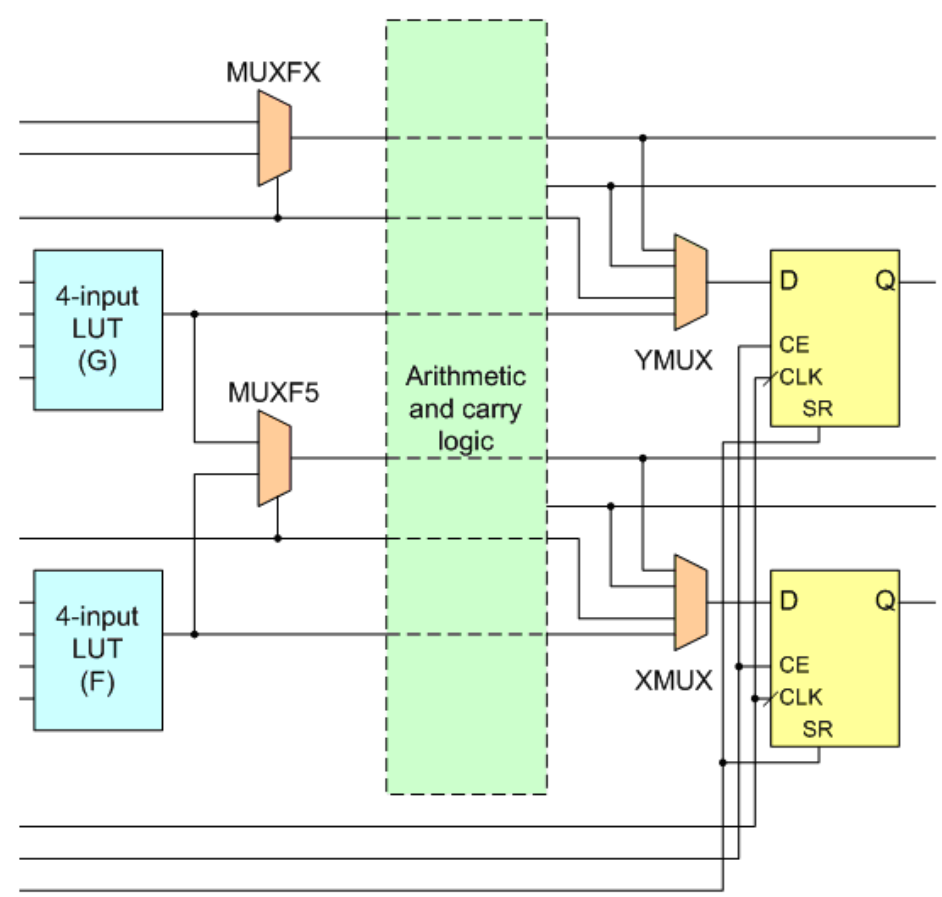

Figure 3.4: CLB architecture for Xilinx Virtex-4 
In our reconfigurable hardware assumption, the connection box is pass-transistor based programmable connection to connect input and output ports of logic blocks to fabricated fixed metal wires. The switch box is multiplexer and pass-transistor based programmable connection to connect the wires. The circuitry for a hardware connection can be obtained by programming logic blocks, connection and switch boxes.

As depicted in Figure 3.5, the reconfigurable hardware assumption is a set of such building blocks. We assume that this reconfigurable hardware is partially run time reconfigurable with no constraints. Among the commercially available FPGAs, with its massive logic resources and partially run time reconfigurable feature, the Xilinx Virtex6 family is a candidate for our reconfigurable computing platform. However, there still exists a constraint of configuring a minimum of 40 CLBs in the same column [43]. Although these FPGAs have useful hardware blocks such as block RAM, digital clock manager and FIFO, only the logic and interconnection resources can be in our model.

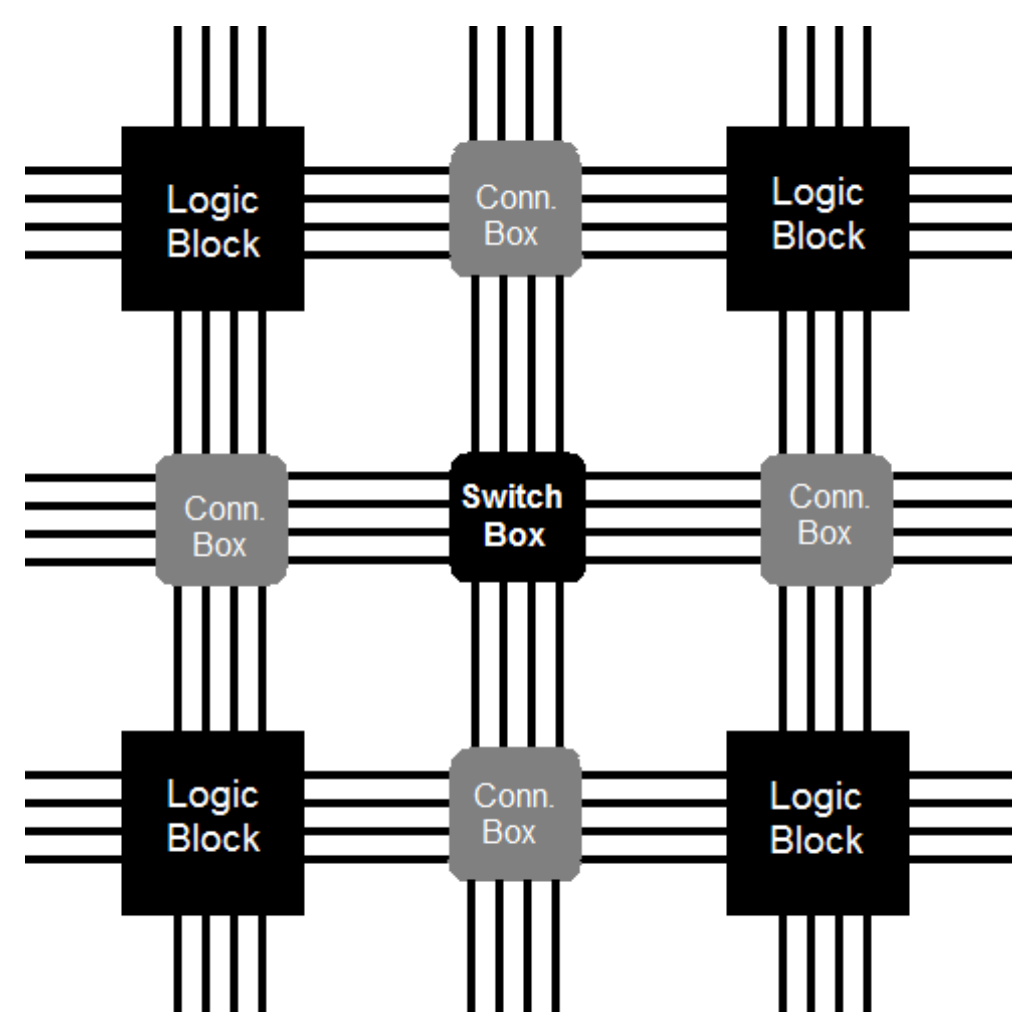

Figure 3.5: Universal reconfigurable hardware assumption 
In this study, the reconfigurable hardware is modeled with a 3-tuple as $R H W(M, N, T)$ where;

- $M$ is the number of logic blocks in a row.

- $N$ is the number of logic blocks in a column.

- $T$ is the reconfiguration time needed for a single logic block. We assume that this time also includes fetching of the bit-stream from external memory.

\subsection{Hardware Task Model}

As mentioned earlier, despite its performance, reconfigurable computing comes with a reconfiguration time penalty during context loading. In contrast to GPP based computing, where task switching requires just saving and changing the program counter and a couple of processor registers, the reconfigurable computing requires bitstream fetching from the external memory and loading it onto appropriate locations on the reconfigurable hardware.

The literature survey on context loading given in Chapter 2 has shown the effect of this reconfiguration time penalty on total execution time. This problem is very similar to switching a task residing in disk drive in an operating system for a conventional computing using virtual memory model.

An embedded real time application requires the preemption of executing task/tasks whenever a higher priority task is scheduled. Such preemption is very simple as far as a software task is concerned. However, for a hardware task such preemption requires reading all flip flop contents (for saving the state of the circuit) and a careful design to stop the execution at a stable point. The same problem exists when a suspended hardware task is rescheduled in writing back the flip-flop contents (for reloading the state of the circuit).

Another limitation of reconfigurable computing may occur if the hardware tasks are too large such that they cannot fit into the reconfigurable hardware. Indeed, there can be a case where more than one hardware task cannot fit and hence no true multitasking is possible. Similar to decreasing reconfiguration time, amount of reconfigurable hardware resources are also increasing, thanks to the enhancements in deep submicron silicon technology. However, selecting a larger device is still not always a solution due to technological limits and increased 
cost in terms of both price and power consumption, which is a key factor in embedded applications.

Finally, as discussed in Chapter 2, existing reconfigurable hardware surface partitioning methods either have low area utilization as a result of fragmentation or require footprint transformation and defragmentation. Due to configuration and flip-flop read/write time, the latter one is not applicable in real time embedded applications.

From the above discussion, it is obvious that the computing platform performance will be degraded if the hardware tasks are given as a single circuitry. The performance become worse if the circuits are arbitrarily shaped. In order to overcome these limitations, we propose a hardware task model, which will minimize the reconfiguration time penalty and increase the reconfiguration device utilization with the help of our complete reconfigurable computing platform components.

As a remedy, the hardware tasks are partitioned into a set of sub-tasks called 'execution blocks' in our hardware task model. As the hardware task is partitioned into smaller execution blocks it becomes possible to decrease the reconfiguration time overhead with our context loading model. Indeed, with the help of our surface partitioning model the fragmentation issue is resolved and high device utilization is achieved.

We define the 'execution block' circuitry as part of the hardware task performing a computation in the most efficient manner. For example an FIR filter or an array multiplier can be considered as an execution block. The execution block is atomic and hence its further partitioning is not feasible.

The reconfiguration time is aimed to be minimized in the literature generally by temporal partitioning of the hardware task into sub-tasks [25,26,27]. In this thesis work, the partitioning process is further extended and spatial parallelism is also taken into consideration. The spatial partitioning process aims to partition the sub-tasks further into smaller sub-tasks. Spatial partitioning process can be applied in two cases.

In the first case, a sub-task circuitry is partitioned into smaller data independent sub-task circuitry. As an example, consider the sub-task performing functionl on data set datal and function 2 on data set data2 in a loop. As shown in Figure 3.6, this sub-task can be partitioned into two sub-tasks each with a loop and a single function. 


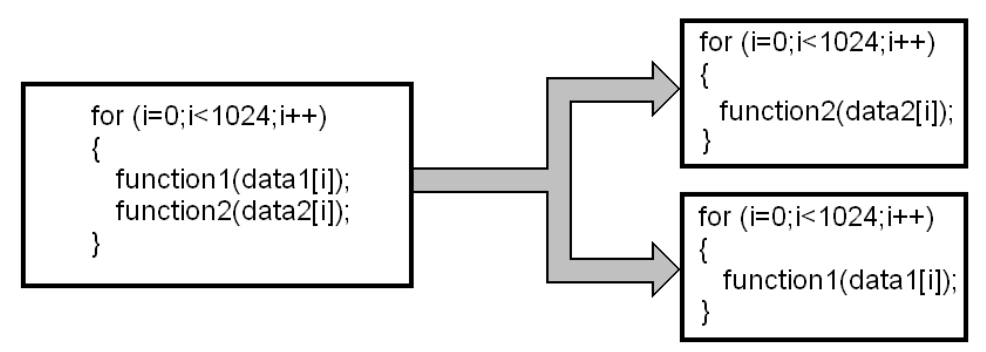

Figure 3.6: Spatial partitioning on the independent data set

If the sub-task circuitry size is too big as compared to other sub-tasks, it is possible to partition sub-task into smaller sub-tasks with data dependency. For instance, assume that a subtask is a loop with a function 3 working on data outputs of function 1 and function 2 is given. As depicted in Figure 3.7, it is possible to partition this sub-task into three subtask with some communication between them. Thanks to our surface partitioning technique and NoC architecture such a partitioning is possible and these partitions can be loaded onto non-contiguous area on the reconfigurable device surface. However, such a data dependent partitioning is feasible as long as the traffic demand is kept at a low level. Otherwise the execution time can suffer from the NoC traffic delays.

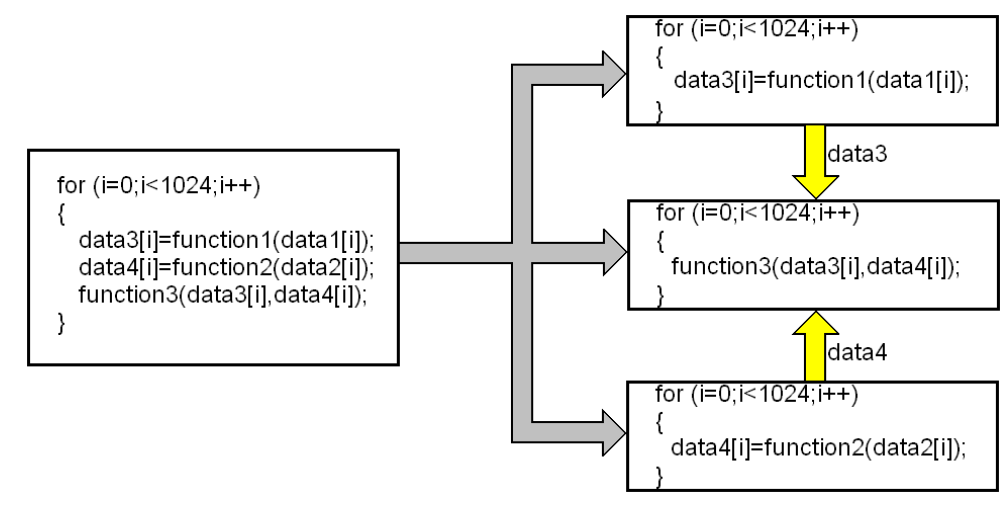

Figure 3.7: Spatial partitioning on the dependent data set

In our computing platform it is the user's responsibility to partition the hardware tasks into execution blocks both using temporal and spatial partitioning. Although this partitioning process is a bit complicated and requires careful analysis of the hardware task, there is no 
restriction on the number of the execution blocks and the execution blocks circuitry can be in any size.

As compared to existing work [19-21], which requires partitioning into fixed sized circuits, we provide a design flexibility to users. Indeed in previous methods, it is the user's responsibility to select a 'good number' for this fixed sub-circuit size, which makes partitioning complicated. Since it is almost impossible to have sub-tasks all having the same size, restricting circuit size to a fixed value will definitely result in fragmentation. Similarly, in multi-tasking systems as the characteristics of tasks are different, the fixed size selection process becomes more problematic.

Our aim is to provide design flexibility similar to the one provided by conventional programming to application programmers. Therefore, in our proposal application programmer is free to choose the size of each execution block. In addition to this flexibility, we aim to achieve much better device utilization compared to fixed size partitioning.

In this study, we model a hardware task with a 3-tuple as $H T\left(S_{E B}, G, D\right)$ where;

- $S_{E B}$ is the set of execution blocks

- $G$ is a directed graph representing the parallelism and dependencies between execution blocks

- $D$ is the deadline of the task to be completed for hard real time tasks.

Also, a level $k$ in the dependency graph $G$ is mathematically modeled as $L_{k}(G)$ and it represent the execution blocks that are obtained by spatial partitioning and operating in parallel.

An example hardware task flow is given in Figure 3.8. The execution of hardware task starts with $E B 0$ followed by $E B 1$, after which three other execution blocks $E B 2, E B 3, E B 4$ will start execution in parallel. Just after the completion of both $E B 3$ and $E B 4, E B 6$ will start execution in parallel with $E B 5$. Finally the hardware task execution will be completed with $E B 7$ that will be executed after the completion of $E B 5$ and $E B 6$. 


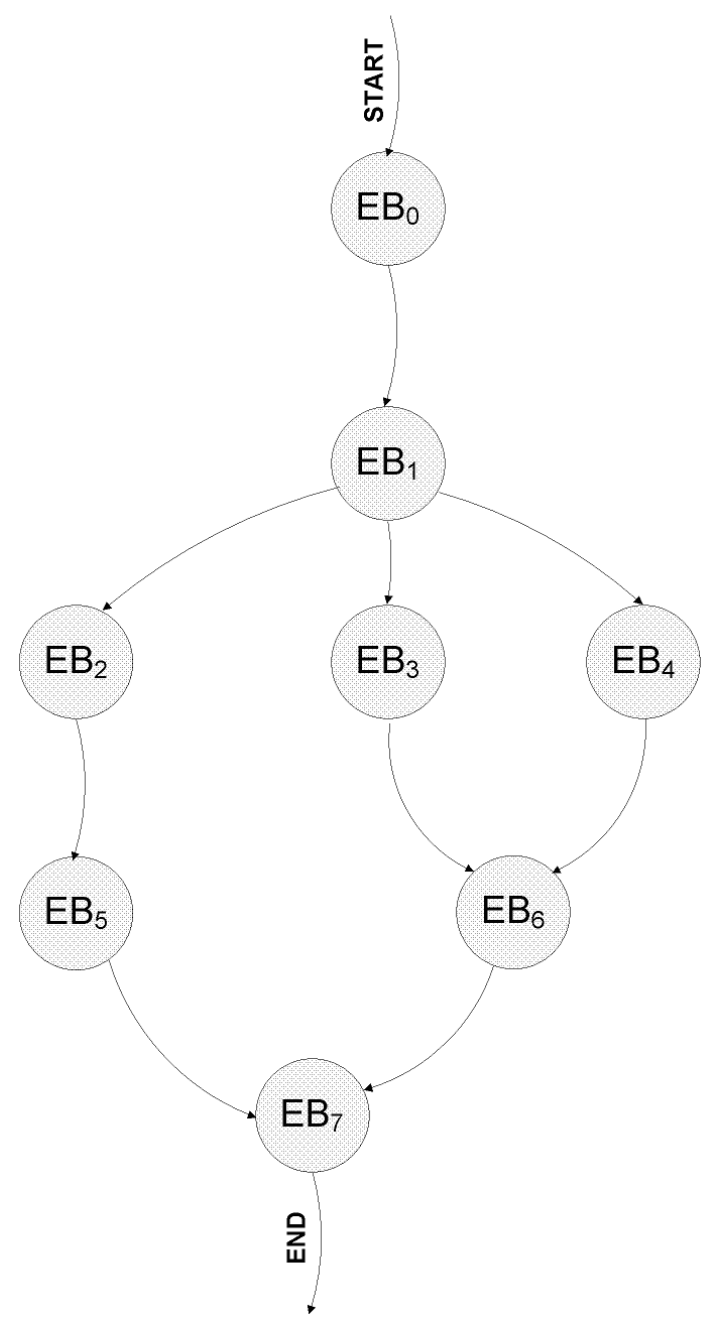

Figure 3.8: An example hardware task dependency graph

The improvements that are achieved with our hardware task model compared to existing works are discussed below:

Reconfiguration time \& task turnaround time Most of the existing works require configuration of all hardware task bit-streams for task switching, however our solution allows a hardware task to start execution just after the first execution block bit-stream is configured on the reconfigurable hardware since the following blocks can now be configured in a pipeline manner. In other words, we minimize the reconfiguration time penalty and achieve a shorter task turnaround time. Assuming that both execution and configuration times are equal and assuming one time unit for all the hardware execution blocks in Figure 3.8, task turnaround time will reduce from 13 to 9 time units. 
Task preemption \& rescheduling With our execution block based hardware task model, it is possible to stop execution of the task at execution block boundaries, i.e. task can be preempted as soon as the currently executing execution block completes its operation. In this way we can achieve 'a soft priority scheduling' with no big time penalty for storing the task context.

Reconfigurable Hardware Utilization \& Device Size In our computing model, it is possible to schedule a task with a size larger than the reconfigurable hardware size and decrease the device cost. Indeed the effective hardware task area is shrunk by reusing the area that was previously allocated to a completed execution block and therefore the device utilization is increased. The wasted area due to fragmentation issues is also kept at minimum by packing more than one execution block into a fixed size reconfigurable hardware area as will be illustrated in the following sections.

\subsection{Execution Block Model}

In our computing model, after partitioning a hardware task into a set of execution blocks, it is the application developer's responsibility to define these execution blocks in a hardware description language (HDL). The rest is handled by our reconfigurable computing platform. Our novel offline bit-stream generation process and hardware operating system will then be responsible to execute the hardware task in the most efficient way.

In order to be compatible with our reconfigurable computing platform, each execution block must have input/output interfaces as depicted in Figure 3.9.

In an execution block, the computation starts with a trigger signal on Start port. The input data for computation is received from system memory over SAC In. If the execution block is obtained by data dependent spatial partitioning, the data can be received from another execution block belonging to the same hardware task over Data In port also. In a similar way, the computational results can be stored back to system memory over SAC Out or sent over Data Out port to another execution block belonging to the same hardware task. Indeed, the SAC In and SAC Out ports are used for operating system services like inter-task communication. Note that if the execution block is not obtained by data dependent spatial partition, then there is no need for Data Out and Data In ports. 


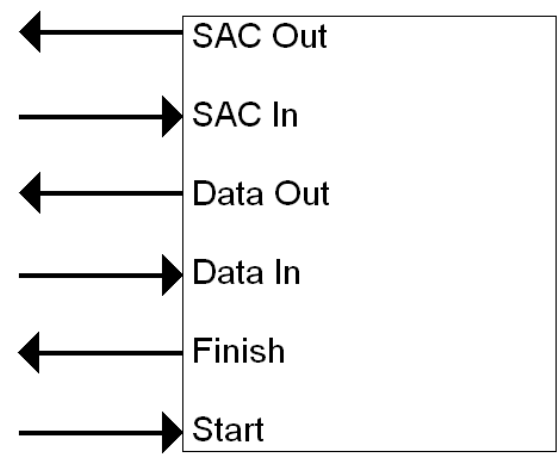

Figure 3.9: An interface template for an execution block

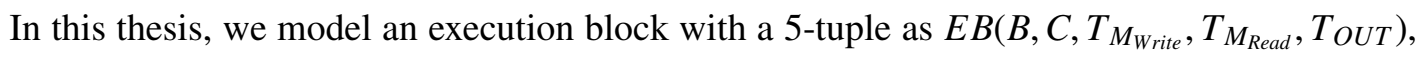
where

- $B$ is number of logic resources needed to implement the execution block

- $C$ is the execution time for the execution block on the target reconfigurable hardware.

- $T_{M_{\text {Write }}}$ is the memory write demand in MByte/s

- $T_{M_{\text {Read }}}$ is the memory read demand in MByte/s

- $T_{O U T}$ is the data generation rate at the Data Out port in MByte/s

\subsection{Surface Partitioning Model}

Reconfigurable hardware surface partitioning technique plays a key role in the overall system performance. It directly affects the underlying reconfigurable hardware utilization and hence has great impact on the required reconfigurable device size, cost and power consumption. Our extensive literature survey and discussion on surface partitioning given in Chapter 2 has shown that, none of the existing surface partitioning methods is directly applicable to our reconfigurable computing platform.

Due to its support for communication media and no time consuming packing or defragmentation requirements, fixed block size partitioning is chosen. Among the fixed block partitioning alternatives, the 1D partitioning suffers from shared and limited bandwidth communication 
media and low operating frequency within partitions due to long wires. Therefore 2D fixed sized surface partitioning technique is our choice. Existing surface partitioning techniques (for example [20-21]) neither model the communication media nor describe how to select the $2 \mathrm{D}$ partitioning parameters. Therefore a novel surface partitioning model specific to our reconfigurable computing platform is developed.

In our partitioning model, we first allocate some fixed sized rectangle regions in a $2 \mathrm{D}$ grid manner as shown in Figure 3.10. These rectangle regions are called 'user block' and they are dynamically reconfigured for hardware task implementation. The rest of the reconfigurable device surface is allocated for communication media and hardware operating system. This area is static and the associated bitstream is loaded at system startup.

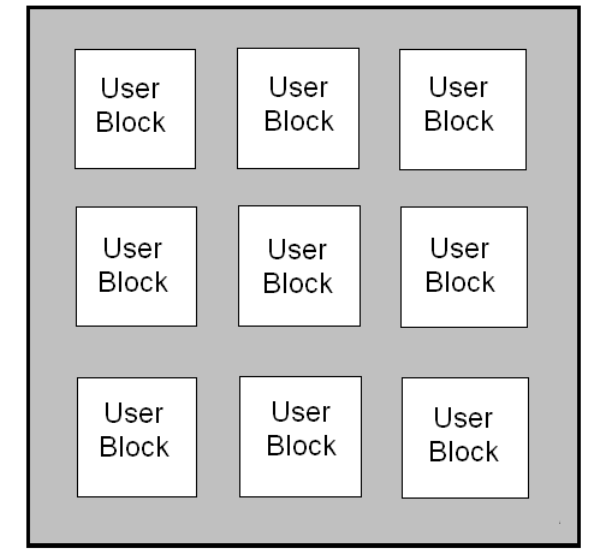

Figure 3.10: $3 \times 3$ user block allocation

The static area, which is called as 'System Area Circuitry (SAC)' in the rest of document, is composed of three function circuitry. First circuitry is used to implement the platform specific 'host\&memory interface'. The second circuitry is the circuitry for the hardware operating system. The third circuitry is the communication media for communication between user blocks and host\&memory interface. In our surface partitioning technique, we reserve the outer area for host\&memory interface and the hardware operating system implementations. The remaining static area is used for the communication media and is called inter block communication network (IBCN) in the rest of this document. Our surface partitioning technique is summarized for a $3 \times 3$ user block partitioning in Figure 3.11.

Thanks to our IBCN architecture, the reconfigurable computing platform is not restricted to 


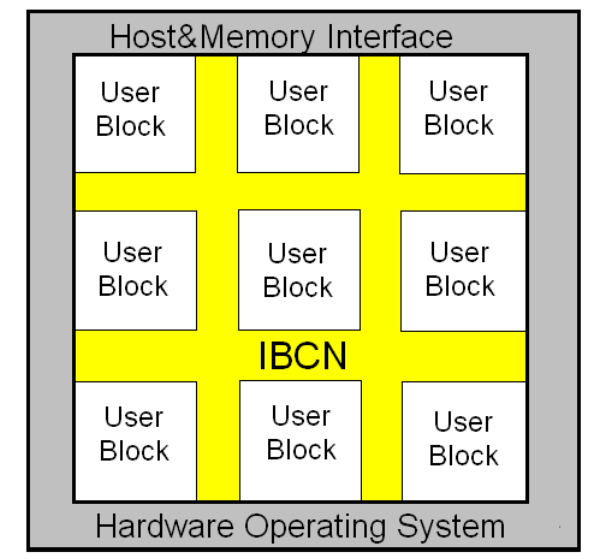

Figure 3.11: Proposed surface partitioning model for $3 \times 3$ user block

use a single reconfigurable device. The IBCN provides seamless communication media for user blocks to access other user blocks and host\&memory interface. Therefore, it is possible to use more than one reconfigurable device with connection over IBCN. With this approach, as shown in Figure 3.12, it is also possible to merge a number of reconfigurable hardware into a single virtual reconfigurable hardware. Such a reconfigurable hardware will be treated as a single reconfigurable device both by the application programmer and hardware operating system.

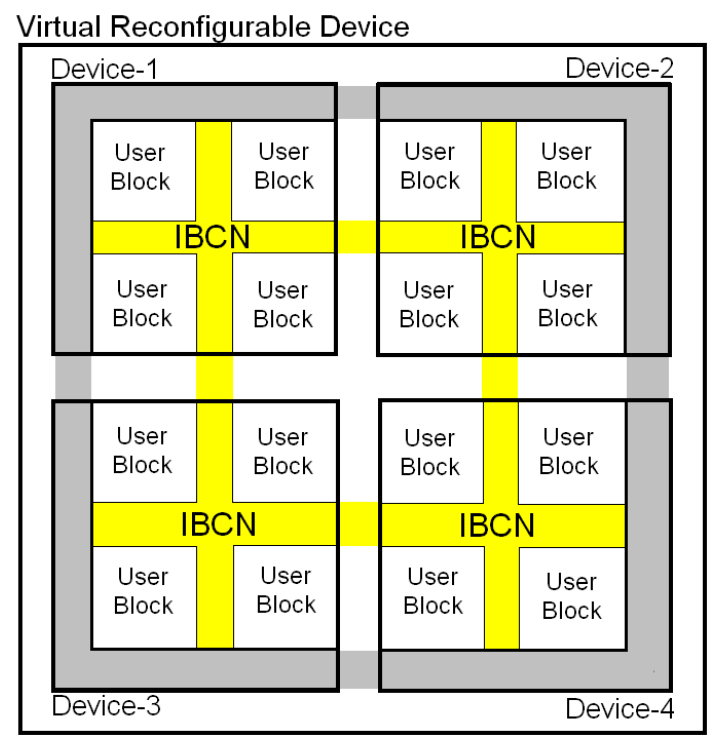

Figure 3.12: Merging example: Four reconfigurable devices merged into a virtual reconfigurable device 


\subsection{Placement Model}

Since we use a fixed block size based partitioning, no external fragmentation problem exists while internal fragmentation is not a big issue due to our novel hardware task model and offline bitstream generation process where the execution blocks of a hardware task can be packed into a number of user blocks. In contrast to existing models $[20,21,26]$, where block size is chosen as equal to the size of the largest hardware task circuitry, we allow hardware tasks to be configured onto more than one user blocks that are not necessarily contiguous.

In our placement model any free user block can be allocated to the task. If there is data connectivity between any two execution blocks, IBCN provides the necessary communication path between these if they are mapped into different user blocks. In order to minimize the affects of the IBCN on execution time, an ad-hoc mapping technique is presented, which will be discussed later in Chapter 6. An example placement for a set of four tasks is shown in Figure 3.13.

\begin{tabular}{|c|c|c|}
\hline \multicolumn{3}{|c|}{ Host\&Memory Interface } \\
\hline $\begin{array}{l}\text { Task1 } \\
\text { UB \#1 }\end{array}$ & $\begin{array}{l}\text { Task2 } \\
\text { UB \#1 }\end{array}$ & $\begin{array}{l}\text { Task3 } \\
\text { UB \#2 }\end{array}$ \\
\hline $\begin{array}{l}\text { Task1 } \\
\text { UB \#2 }\end{array}$ & $\begin{array}{l}\text { Empty } \\
\text { UB }\end{array}$ & $\begin{array}{l}\text { Task4 } \\
\text { UB \#2 }\end{array}$ \\
\hline & IBCN & \\
\hline $\begin{array}{l}\text { Task3 } \\
\text { UB \#2 }\end{array}$ & $\begin{array}{l}\text { Task4 } \\
\text { UB \#1 }\end{array}$ & $\begin{array}{l}\text { Task1 } \\
\text { UB \#2 }\end{array}$ \\
\hline
\end{tabular}

Figure 3.13: A placement example: surface partitioning for a set of four tasks

\subsection{Reduced Hardware Task Model}

In our reconfigurable computing platform, a hardware task is defined in terms of a set of execution blocks, a dependency graph and a deadline. The user is free to have different size execution blocks. With the help of our offline packing process, we will convert a hardware task into a set of equal sized user blocks and call the resulting circuit as 'reduced hardware 
task'.

For a given hardware task $H T$, we model the reduced hardware task with the 3-tuple as $H T^{\prime}\left(S_{U B}, G^{\prime}, D\right)$ where,

- $S_{U B}$ is a set of user blocks

- $G^{\prime}$ is a directed graph representing the parallelism and data dependencies between user blocks

- $D$ is the deadline for its completion of execution.

An example hardware task and its corresponding reduced hardware task (b) are shown in Figure 3.14. Note that in this example, the reduced hardware task is obtained by packing execution blocks into user blocks with size of $40 \mathrm{~K}$ logic blocks.

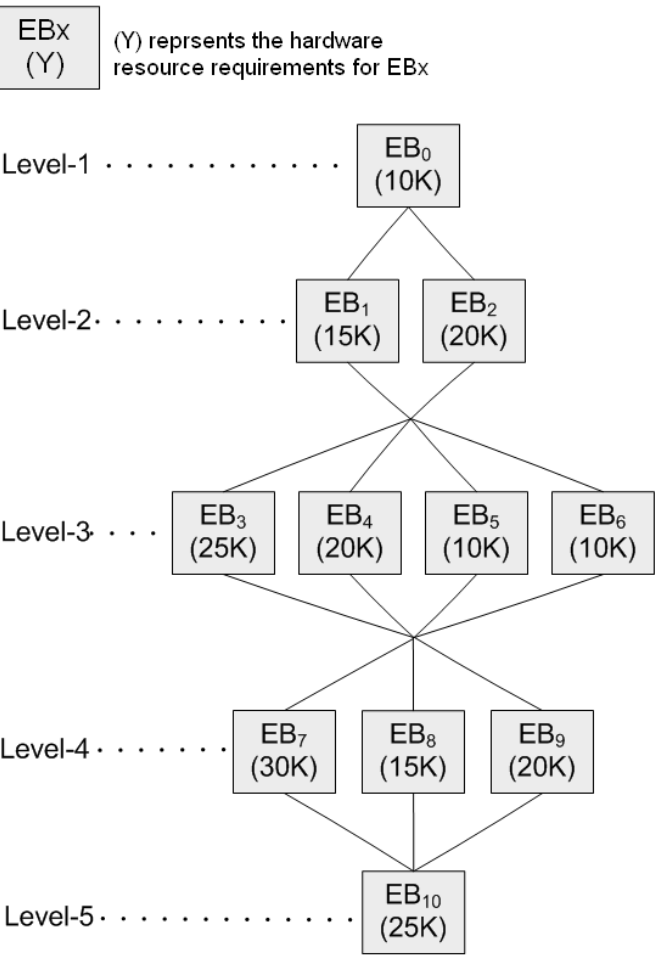

(a)

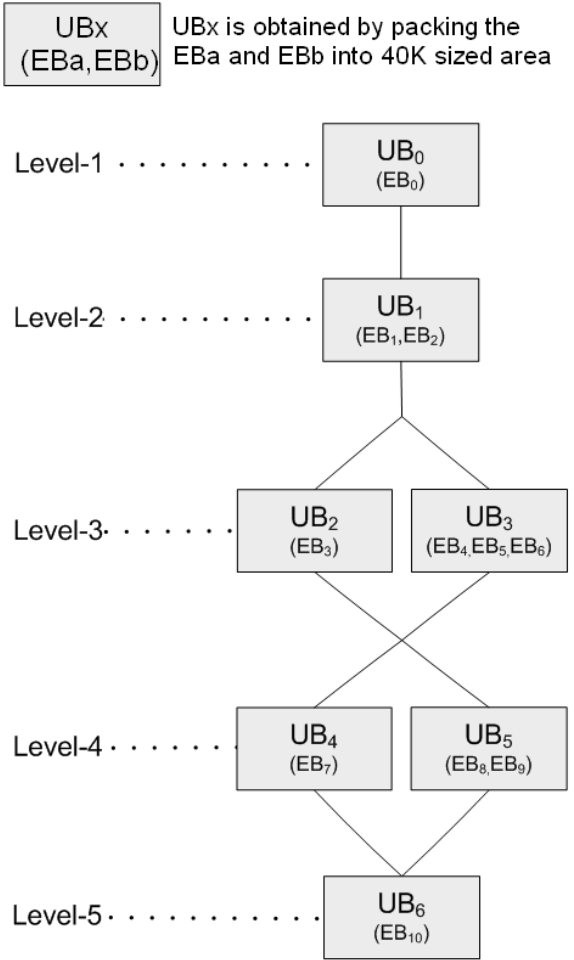

(b)

Figure 3.14: a) An example hardware task b) its reduced hardware task 


\subsection{Context Loading Model}

As was stated earlier, our reconfigurable computing platform aims to provide short turn around time for scheduled hardware tasks. The task turn around time is composed of execution time and reconfiguration time. In this thesis work, we used the following context loading scheme to minimize the task turn around time whenever a hardware task is scheduled by the user:

Step-1 (Initialization) Starting from the one with the largest execution time, load all user blocks (UBs) in the first level of reduced task dependency graph $L_{1}\left(G^{\prime}\right)$. Whenever a user block is loaded, it immediately starts execution in this initialization step.

Step-2 Just after all user blocks are loaded for the first level, continue loading the ones in the second level $L_{2}\left(G^{\prime}\right)$ and set $k=1$.

Step-3 Wait till all user blocks on $L_{k}\left(G^{\prime}\right)$ complete their execution. Set $k=k+1$.

Step-4 If all user blocks on $L_{k}\left(G^{\prime}\right)$ are already loaded, start user blocks on $L_{k}\left(G^{\prime}\right)$ and go to Step-6.

Step-5 Wait till all user blocks on $L_{k}\left(G^{\prime}\right)$ are loaded and start user blocks on $L_{k}\left(G^{\prime}\right)$ (prefetching).

Step-6 Start loading user blocks in level $L_{k+1}\left(G^{\prime}\right)$.

Step-7 Go to Step-3 if end of $G^{\prime}$ is not reached.

Using the above context loading scheme, the execution time can be locally minimized by executing user blocks that have no data dependency in parallel. The reconfiguration time is minimized by prefetching the next level user blocks while current level user blocks are being executed. 


\section{CHAPTER 4}

\section{USER BLOCK SIZE SELECTION}

In our hardware task model, the execution blocks are application dependent and can be in different sizes. Since we pack execution blocks of a task into a number of fixed size user blocks, it is possible to have some empty spaces in the user block. This empty space can be considered as internal fragmentation and it can decrease the overall reconfigurable hardware utilization if the user block size is not properly selected. Similarly, the IBCN circuitry is implemented with the resources of the reconfigurable hardware and the area reserved for IBCN should be kept optimal for a high level of device utilization while providing the necessary communication paths. Since the area needed for IBCN is directly proportional to the number of user block, the surface partitioning should be done carefully in order not to waste too much space for IBCN implementation.

In this dissertation, we address embedded applications for which it is possible to find an optimal user block size because we have the knowledge of all hardware tasks and execution blocks prior to field operation. For better device utilization, we introduce the following offline optimization process to find the $2 \mathrm{D}$ block partitioning parameters.

Part of the reconfigurable hardware surface is used for static SAC, which is composed of host\&memory interface and hardware operating system circuits. In the rest of the thesis, we model the SAC with the ordered pair as $S A C\left(M_{S A C}, N_{S A C}\right)$ where;

- $M_{S A C}$ is the number of outer vertical logic blocks used for SAC.

- $N_{S A C}$ is the number of outer horizontal logic blocks used for SAC.

The implementations of the host\&memory interface and hardware operating system are plat- 
form dependent and not affected by the user block size selection. Therefore $M_{S A C}$ and $N_{S A C}$ values are considered as constant for the user block size selection process.

Similar to SAC implementation, the IBCN implementation requires some surface area on the reconfigurable hardware. But the size of this area depends on the user block size. When the reconfigurable hardware is partitioned into $m \times n$ user blocks, we model the communication path with the four-tuple as $\operatorname{IBCN}\left(m, n, M_{I B C N(m, n)}, N_{I B C N(m, n)}\right)$ where;

- $m$ is the number of user blocks in horizontal axis.

- $n$ is the number of user blocks in vertical axis.

- $M_{I B C N(m, n)}$ is the number of total logic blocks needed for IBCN in horizontal axis.

- $N_{I B C N(m, n)}$ is the number of total logic blocks needed for IBCN in vertical axis.

Using the above circuit models, the user block size (UBS) then becomes;

$$
U B S=\left\{\frac{\left(M-M_{S A C}-M_{I B C N(m, n)}\right)}{m}\right\} \times\left\{\frac{\left(N-N_{S A C}-N_{I B C N(m, n)}\right)}{n}\right\}
$$

In our surface partitioning technique, the selection of partitioning parameters is important in determining the efficiency of device usage. In Figure 4.1a, a reconfigurable device with a size of $M \times N$ logic blocks and in Figure $4.1 \mathrm{~b}$ a hardware tasks containing a set of execution blocks are given. Note that, the given execution blocks are composed of a number of logic blocks (Figure 4.1b). After allocating outer $N_{S A C}$ logic blocks in horizontal axis and $M_{S A C}$ logic blocks in vertical axis for SAC implementation, the remaining parts are partitioned into $m \times n$ user blocks and a predefined area is reserved for IBCN switch implementation for each user block (Figure 4.1c). With the completion of the partitioning process, for each hardware task, the execution blocks are packed into user blocks. In order to achieve higher device utilization, the partitioning parameters $m$ and $n$ must be chosen in such a way that the following figures of merits are both maximized.

- utilization of the user area $\rho_{\text {user }}$, which is the ratio of 'gray area' to 'all minus SAC area' (Figure 4.1c). 
- utilization of the area within user blocks $\rho_{\text {internal }}$, which is the ratio of area filled by execution blocks to user block size after the execution block packing process (Figure 4.1d).

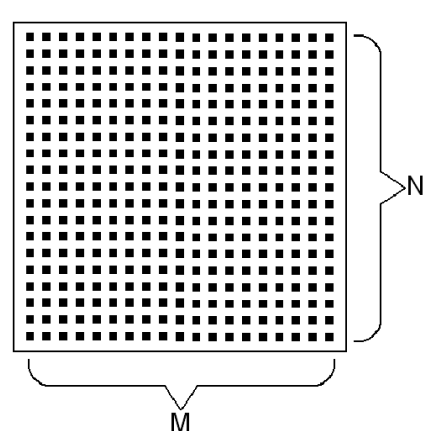

(a)

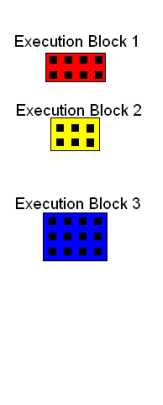

(b)

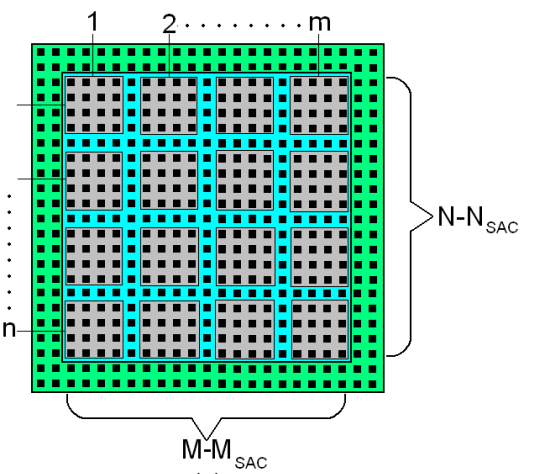

(c)

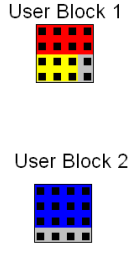

(d)

Figure 4.1: Problem of user block size selection

\subsection{Problem Formulation}

Given;

- a reconfigurable hardware, $R H W(M, N, T)$ where

- $M$ is the number of logic blocks in a row.

$-N$ is the number of logic blocks in a column.

- $T$ is the reconfiguration time needed for a single logic block.

- an SAC implementation, $S A C\left(M_{S A C}, N_{S A C}\right)$ where

- $M_{S A C}$ is the number of outer vertical logic blocks used for SAC.

- $N_{S A C}$ is the number of outer horizontal logic blocks used for SAC.

- a set $Y$ of hardware tasks where

- a hardware task $j$ is modeled as $H T_{j}\left(S_{E B_{j}}, G_{j}, D_{j}\right)$ including the set of execution blocks $S_{E B_{j}}$, dependency graph $G_{j}$ and a deadline $D_{j}$ 
- an execution block $i$ in $H T_{j}$ is modeled as $E B_{i, j}\left(B_{i, j}, C_{i, j}\right)$, where $B_{i, j}$ is the number of logic resources needed to implement the execution block $i$ of hardware task $j$ and $C_{i, j}$ is the execution time for the execution block $i$ of hardware task $j$ on the specific reconfigurable hardware.

- a level $k$ in the task dependency graph $G_{j}$ is modeled as $L_{k}\left(G_{j}\right)$ and it represent the execution blocks that can be executed in parallel in hardware task $j$.

- user provided device utilization factor $\beta_{j}$ for each hardware task $j . \beta_{j}$ value should be selected within the range $[0,1]$ and for a task that will be executed only once, $\beta_{j}$ should be small, but for periodic tasks it should be large. Choosing an improper $\beta_{j}$ value may result in degraded reconfigurable hardware utilization.

Problem $P$ is to find $m$ and $n$, i.e. the number of user blocks in horizontal and vertical dimensions respectively such that when the reconfigurable hardware is divided into $m \times n$ user blocks, total reconfigurable hardware utilization, $\rho_{\text {total }}$, is maximized. Hence;

Problem $P: \max \left\{\rho_{\text {total }}\right\}$

subject to

$$
\forall j \in Y, \forall i \in S_{E B_{j}}: \quad M \times N \geq U B S \geq \max \left(B_{i, j}\right)
$$

$$
\forall j \in Y, \forall k \in G_{j}: \quad \text { effective_size }\left(H T_{j}\right) \geq \max \left(\sum_{i \in L_{k}\left(G_{j}\right)} B_{i, j}+\sum_{i \in L_{k+1}\left(G_{j}\right)} B_{i, j}\right)
$$

where

- $\rho_{\text {total }}=\rho_{\text {user }} \times \rho_{\text {internal }}$

- $\rho_{u s e r}=\frac{m \times n \times U B S}{\left(M-M_{S A C}\right) \times\left(N-N_{S A C}\right)}$ which represents the ratio of area usable by user hardware tasks to total available area

- $\rho_{\text {internal }}=\frac{\sum_{j \in Y}\left(\rho_{\text {internal }_{j}} \times \beta_{j}\right)}{\sum_{j \in Y} \beta_{j}} \quad$ which represents the average internal utilization

- $\rho_{\text {internal }}=\frac{\text { size_of }\left(H T_{j}\right)}{\text { effective_size }\left(H T_{j}\right)} \quad$ which represents the internal utilization for hardware task $j$ 
- size_of $\left(H T_{j}\right)=\sum_{i \in S_{E B_{j}}} B_{i, j} \quad$ which represents the sum of logic requirements for execution blocks within the set $S_{E B_{j}}$ for hardware task $j$

- effective_size $\left(H T_{j}\right)=r_{j} \times U B S$ which represents the maximum logic resources needed for hardware task $j$ during its execution

- $r_{j}$ is the maximum number of user blocks allocated to hardware task $j$ by the scheduling mechanism at any instance

- $U B S=\left\{\frac{\left(M-M_{S A C}-M_{I B C N(m, n)}\right)}{m}\right\} \times\left\{\frac{\left(N-N_{S A C}-N_{I B C N(m, n)}\right)}{n}\right\}$

- $m, n \in\{1,2,3, \ldots\}$

Note that the computation of the effective_size $\left(H T_{j}\right)$ and hence $r_{j}$ of a hardware task $j$ depends on the hardware task dependency graph $G_{j}$ and user block size $U B S$ and therefore requires another optimization problem.

Finding effective_size $\left(H T_{j}\right)$ can be modeled as a subproblem $P_{1}$ as follows:

Given

- a hardware task $H T_{j}$ with a set of execution blocks $S_{E B_{j}}$ and dependency graph $G_{j}$

- the user block size $U B S$

Problem $P_{1}: \min \left\{r_{j}\right\}$

subject to

$$
\forall j \in Y, \quad \forall k \in G_{j}^{\prime}: \quad r_{j}=\max \left\{\text { noub }_{k}+\text { noub }_{k+1}\right\}
$$

$$
\forall j \in Y, \quad \forall i \in S_{E B_{j}}: \quad 1=\sum_{k \in S_{U B_{j}}} x_{i, k}
$$

$$
\forall j \in Y, \quad \forall i \in S_{E B_{j}}, \text { forallk } \in S_{U B_{j}}: \quad x_{i, j} \in\{0,1\}
$$

$$
\forall j \in Y, \quad \forall k \in S_{U B_{j}}: \quad U B S \geq \sum_{i \in S_{E B_{j}}} x_{i, k} \times B_{i, j}
$$




$$
\forall j \in Y, \quad r_{j} \in\{1,2,3, \ldots\}
$$

where

- $\operatorname{noub}_{k}$ is the number of user blocks at level $k$ of $G_{j}^{\prime}$;

- $x_{i, k}$ is a binary variable defined as follows:

$$
x_{i, k}= \begin{cases}1 & \text { if } E B_{i} \text { is packed in } U B_{k} \\ 0 & \text { otherwise }\end{cases}
$$

In the above optimization problem constraint (4.1) indicates that the user block size $U B S$ must be at least the size of the largest execution block in the system. This is because the execution blocks are atomic and the whole circuitry for one execution block must be loaded onto the same user block. Constraint (4.2) appears in our formulation due to our heuristic approach on context loading process (see Section 3.8) that is used to minimize the task turn around time. This constraint guarantees for each hardware task $j$ that at least an area equal to the maximum, taken over all levels, of the sum of execution block sizes in two consecutive levels of the dependency graph $G_{j}$ is allocated for execution without any time penalty for reconfiguration. In a similar way, constraint (4.3) indicates that the number of user blocks needed for a task $j$ should be equal to the sum of the number of user blocks in two consecutive levels of reduced hardware task graph $G_{j}^{\prime}$. Constraints (4.4) and (4.5) guarantees that an execution block is atomic and can be packed into one user block only. Finally, the sum of logic requirements of execution blocks packed into the same user block cannot be larger than UBS (constraint 4.6).

\subsection{Problem Analysis}

For the optimization problem $P$, the quality of the solution strongly depends on the $\rho_{\text {internal }}$ value calculated for a given $U B S$. Because for the given hardware tasks and $U B S$ maximizing $\rho_{\text {internal }}$ is an objective of our second optimization problem $P_{1}$. In our proposal, instead of loading just a single execution block to a user block on the reconfigurable harware surface, we propose to pack execution blocks working in parallel into user blocks. In this way the $r_{j}$ value, hence effective_size $\left(H T_{j}\right)$, can be minimized. 
Since the execution blocks are atomic and it is not feasible to partition them further into smaller units, we have to pack these different sized execution blocks into fixed sized user blocks having size of $U B S$. This packing process is known as 'one dimensional bin-packing problem (BPP-1)' in the literature. Coffman et al. have shown that one dimensional binpacking problem is NP-complete [44]. Since part of optimization process $P_{1}$ is NP-complete, then our optimization problem P is NP-complete too.

For a given $U B S$ and hardware task $j$, calculation of $\rho_{\text {internal }}$ requires the solution of BPP-1 for all execution blocks on the same level in $G_{j}$. The BPP-1 is a well studied topic in the literature and some major works are discussed below.

\subsection{Literature survey on BPP-1}

Since BPP-1 is NP-Complete, mostly heuristics techniques were proposed to find near optimal solutions. Jatinder et al. [45] introduced a heuristic based on minimum bin slack (MBS) and it has been shown that this method finds optimal solutions if the sum requirements of items is less than or equal to twice the bin capacity.

In [46] an exact solution procedure for BPP-1 was proposed. This technique, called BISON, is composed of different bound arguments and reduction procedures, several heuristics including tabu search and a branch and bound procedure. It is shown that BISON finds exact solution for most of the time.

Flezsar and Hindi [47] have proposed several heuristic methods also. Some of the proposed heuristics are based on MBS and the variable search results. Computational results have shown that the most effective algorithm was the one using the MBS to generate initial solutions and using variable neighborhood search (VNS) method to find exact solutions based on generated initial solution. The authors reported that this technique found optimal solutions for 1329 instances and finds 4 better solutions than best known out of 1370 well known benchmark.

A hybrid heuristic called HI-BP was proposed by Alvim et al. [48]. In this method a combination of lower bounding strategies, the generation of initial solutions by the dual min-max problem, the use of load redistribution based on dominance, differencing, and unbalancing; 
and the inclusion of an improvement process utilizing tabu search methods were combined to achieve better results. Computational results have shown that the proposed hybrid method generates better results compared to BISON and VNS method.

In 2006, a weight annealing based heuristics was proposed by Loh [49]. Starting from an initial solution obtained with first-fit decreasing methods, weights are assigned to distort sizes according to the packing solutions of individual bins. Then a local search was carried out to swap the items. Iteratively this weighting and local search procedure was carried out with a cooling schedule. The proposed technique was applied to both BBP-1 and BBP-2 problems and it has been reported that with its $O\left(n^{3}\right)$ complexity, this technique gives the best result among existing methods.

In our computing platform, the bin packing decision is given during our offline bitstream generation process. Therefore instead of designing a new BBP-1 solver, we selected the existing weight annealing based heuristics [49] as our BBP-1 solver and used it for the solution of subproblem $P_{1}$ in our model.

\subsection{Greedy Heuristic for User Block Size Selection}

Since optimization problem $P$ is NP-complete, we seek a sub-optimal solution in bounded time. Our greedy method starts with an initial solution, where $U B S$ is equal to the whole reconfigurable device size. With such a $U B S, \rho_{\text {user }}$ will be maximized but $\rho_{\text {internal }}$ will have the worst value. We then decrease $U B S$ by increasing $m$ and then $n$ values at each iteration such that the user block will be in a square like shape. This iterative process decreases $\rho_{\text {user }}$, increases $\rho_{\text {internal }}$ and continues till there is no improvement in $\rho_{\text {total }}$.

The proposed heuristic finds a good but non-optimal solution for $m$ and $n$ values when it terminates. Another termination criterion is that $U B S$ should at least be equal to the size of the largest execution block, $E B_{\max }$, in the given hardware tasks list. Calculation of $\rho_{\text {user }}$ requires finding effective circuit size and hence bin packing of execution blocks at the same level of the dependency graph into user blocks. The pseudo code of our greedy heuristic proposal and the pseudo code for finding effective circuit size are given in Figure 4.2 and Figure 4.3 respectively. 


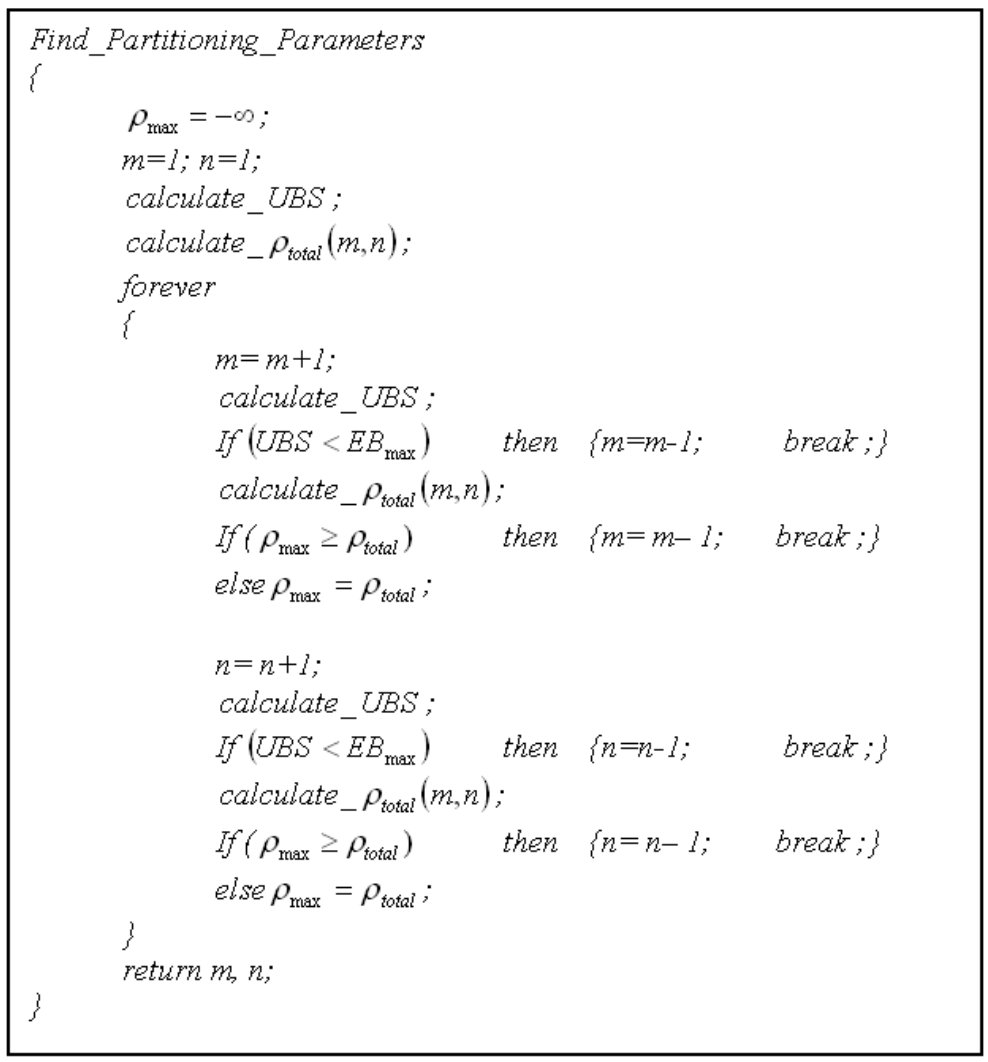

Figure 4.2: Pseudo code for greedy heuristic user block size selection 


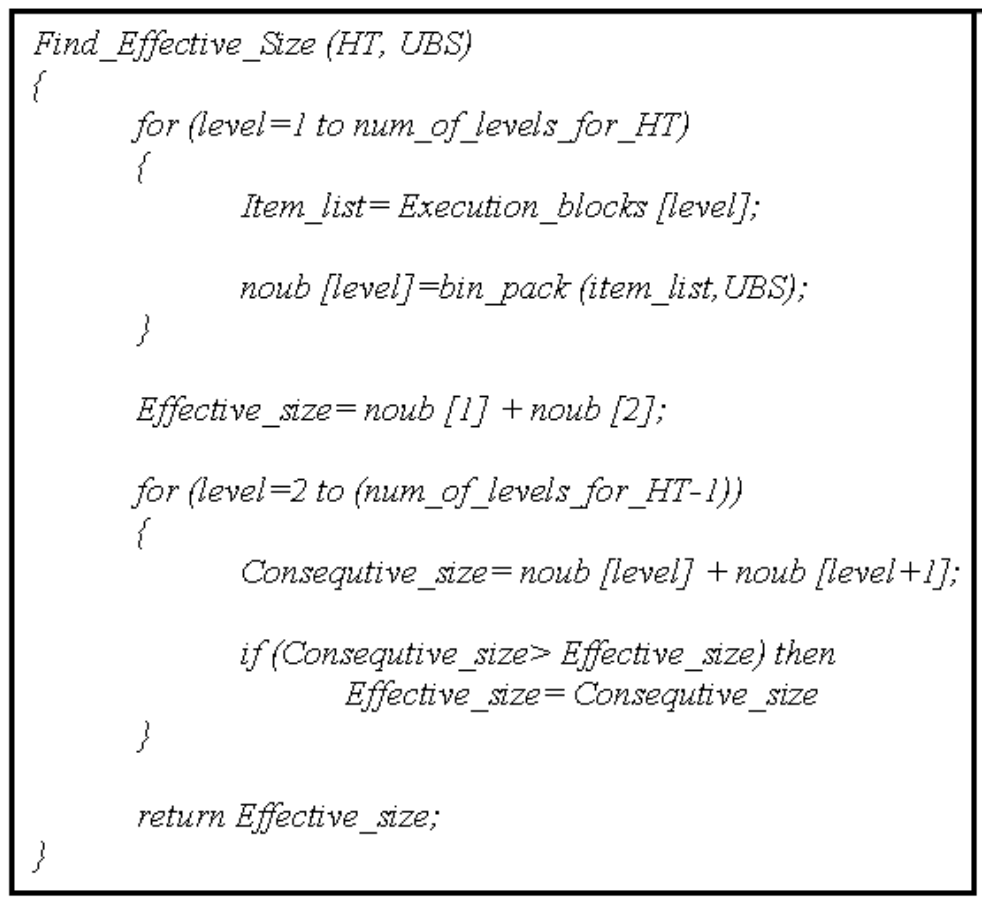

Figure 4.3: Pseudo code for finding effective circuit size

\subsection{Quality Analysis for Greedy Heuristic}

As was stated earlier, the NP-completeness of our optimization problem $P$ is due to having BPP-1 as its sub-problem. In order to show how well our greedy heuristic is, a comparison using a relaxed upper bound has been performed. The relaxed upper bound is obtained by removing constraint (4.4) that guarantees the execution blocks are to be atomic. In this new relaxed sub-problem, there is no need to solve BPP-1 and the number of user blocks needed to pack execution blocks at level $k$ in $G$ becomes simply;

$$
\text { ceil }\left\lceil\frac{\sum_{i \in L_{k}\left(G_{j}\right)} B_{i, j}}{U S B}\right\rceil
$$

With this relaxation the complexity of $P_{\text {relaxed }}$ becomes $O\left(n^{3}\right)$. The exact solution of $P_{\text {relaxed }}$ now provides an infeasible solution and an upper bound for the optimal and can therefore be used to judge the quality of our heuristic solution.

In order to find the average gap between solutions provided by our proposed greedy heuristic and $P_{\text {relaxed }}$ solution, a simulation study has been carried out. In these simulations, 100 test cases are created by randomly generating 1000 hardware tasks for each case and the 
number of execution blocks are selected between 100 and 200 randomly. Similarly, the execution block sizes are randomly selected in the interval $[10 K-40 K]$ logic blocks and the dependency graph depth is randomly selected between 3 and 10 . The device under study is assumed to be 5 million logic block equivalent and the area wasted for IBCN is modeled as $8 K \times$ number of user blocks. The simulation result has shown that the gap between our greedy heuristic and the relaxed upper bound for $\rho_{\text {total }}$ value is around $4.5 \%$ with a confidence interval of $97 \%$. 


\section{CHAPTER 5}

\section{INTER BLOCK COMMUNICATION NETWORK ARCHITECTURE}

In the proposed reconfigurable hardware surface partitioning methodology, the area between the user blocks are dedicated for inter block communication network (IBCN). Since our execution packing process can pack execution blocks that operates in parallel but have data decency into different user blocks, IBCN is then responsible for providing this data path. In a similar manner, the memory access demands of user blocks are handled by IBCN. Finally, IBCN is also the communication media for high level operating system services and inter-task communication between hardware tasks and software task. In this thesis work, a novel Network on Chip (NoC) architecture is designed to provide the necessary support as IBCN. In this chapter, first the communication requirements on our IBCN are analyzed. Then an extensive literature survey is given for existing NoC architectures. Finally, the proposed architecture is presented in detail with all its building blocks.

\subsection{Communication Requirements}

In our reconfigurable hardware platform, IBCN is responsible to provide communication and data path between;

- user blocks of a hardware task (for handling data dependency)

- user blocks belonging to different hardware tasks (for inter-task communication)

- user blocks and host processor (for inter-task communication between software task and hardware tasks) 
- user blocks and host processor (for high level operating system service)

- user blocks and external memory (for memory read and write access)

- user blocks and hardware operating system (for task management and high level operating system services).

Below, these communication requirements are analyzed in detail.

\subsubsection{Task Level Communication Requirements}

In the proposed reconfigurable computing platform, it is required to partition a hardware task into a set of execution blocks. This partitioning process aims improving device utilization and reconfiguration time overhead. In this way, it is possible to synthesize, place and route the execution blocks individually in a much more efficient way as compared to whole hardware task. Also, the operating frequency of execution blocks will be much faster than the whole hardware task due to possible long wire delays in reconfigurable devices. Finally, it may now be possible to obtain optimized library for execution blocks in a much shorter design time.

Despite these advantages, some new communication requirements arise when a whole hardware task is partitioned into execution blocks. If the execution blocks having data dependencies are packed into different user blocks, then the DATA PATH traffic demand arises between user blocks. The other traffic requirement is the control signals between hardware operating system and the user blocks. A TRIG SIGNAL must be sent from the hardware operating system when it is time to start the execution of a user block according to reduced hardware task dependency graph. In a similar fashion, a FINISH SIGNAL must be sent to hardware operating system by the user block completing its execution.

\subsubsection{Memory Access Requirements}

Unified memory architecture is used in our reconfigurable computing platform. Although it is possible to implement this memory on the reconfigurable hardware, it will consume too much logic blocks and will operate at slower frequency than commercially available memory devices. Indeed, this memory should be accessible by the GPP also. Therefore, although it is not mandatory, an external memory is assumed for IBCN discussion. However, it is still 
possible to implement a small sized low latency access register file for each execution block depending on its local memory requirement.

In our model, execution blocks can perform memory read and memory write individually. Therefore, there will be more than one memory access requirement at the same time. In the proposed IBCN architecture, these memory access requirements are routed to system area circuitry, which is responsible for physical memory access. The memory writes will be a posted message with data, i.e. execution block will not wait till the data is written into memory and it requires $M_{-}$WRITE message to be sent from the execution block to system area circuitry. The memory read will be a REQUEST message from execution block to system area circuitry and later followed by an $M_{-} R E A D$ message with data from the system area circuitry.

\subsubsection{Operating System Services Communication Requirements}

In a multi-tasking computing system, there is a need for data exchange and synchronization between tasks, i.e., there is a need for inter-task communication. In general such a communication between tasks are handled using operating system services like semaphores, pipes, sockets and mailboxes. All of these inter-task communication methods require system calls to operating system kernel. In a similar fashion, high level operating system services like file system and networking requires system calls.

In our reconfigurable computing platform these communication demands for software task is handled by the commercial operating system. In case of a communication request between a software task and hardware task; the commercial operating system and both parts of our hardware operating system are working together. The communication of the hardware task starts with the SYS_CALL message from hardware task to the hardware operating system circuitry on the reconfigurable hardware. This message is forwarded to the software part of the hardware operating system over host interface and necessary action is performed there. For a communication request from the software task, the reverse path is fallowed and the hardware operating system circuitry sends $T A S K_{-} C A L L$ message to the target user block.

The communication demand between two hardware tasks is handled in a similar fashion. SYS_CALL messages are sent to hardware operating system circuitry and responses are received via TASK_CALL messages. Although it is possible to have a peer to peer communica- 
tion between two hardware tasks over IBCN, the communication demand between hardware tasks is also handled over our hardware operating system circuitry. Because, in some cases the target hardware task can be waiting in the queue for context loading or the execution of target hardware task does not reach yet to a point to handle this communication. Indeed, for peer to peer communication a register needed in the hardware tasks to keep the physical location of the receiver hardware task. This requires extra hardware area and should be updated if the receiver hardware task is scheduled later than the sender hardware task.

The above discussion on the communication requirements are summarized in Table 5.1.

Table 5.1: IBCN message types and their characteristics

\begin{tabular}{llllll}
\hline Name & Source & Destination & Size & Priority & Frequency \\
\hline START SIGNAL & SAC & User Block & Short & High & Low \\
FINISH SIGNAL & User Block & SAC & Short & High & Low \\
DATA PATH & User Block & User Block & Long & High & Moderate \\
SYS_CALL & User Block & SAC & Short & Low & Low \\
TASK_CALL & SAC & User Block & Short & Low & Low \\
REQUEST & User Block & SAC & Short & High & High \\
M_WRITE & User Block & SAC & Long & High & High \\
M_READ & SAC & User Block & Long & High & High \\
\hline
\end{tabular}

\subsection{Literature Survey on NoC}

The 2D fixed block base reconfigurable hardware surface partitioning technique with the idea of using the area between blocks for communication media has been discussed in a number of studies $[20,21,26]$. However, these studies were lack of information about the architecture and implementation of this communication media. On the other hand, our IBCN functionality is very similar to 'network on chip (NoC)' functionality in 'system on chip (SoC)' architectures. The NoC is a well studied subject in literature and below, some major works targeting 2D mesh topologies are discussed briefly.

With the advances in silicon process technology, it becomes cheaper to build SoC devices with a number of processor cores, storage units and external peripheral interfaces. Since the time-to-market is a critical issue, proven intellectual property (IP) cores are used in SoC design. However, IP's come with its own connectivity interface and have different clock 
frequency demands. In addition, with deep-submicron processes the long wires became a limiting factor for high speed operation. As a remedy, the NoC architecture was used for connectivity between IP cores instead of shared bus architecture with fixed frequency. The NoC architectures are packet switching networks with different routing and packet structures.

One of the earliest NoC architecture was a 2D folded torus NoC architecture [50]. For a $4 \times 4$ tile based SoC with processors and DSPs, the physical design and bandwidth problems of the shared bus architecture were solved with just $6.6 \%$ silicon area overhead for interconnect network. In the proposed network, a 5-port router was used with pre-scheduled virtual channel for static traffic and cyclic reservation for dynamic traffic.

In literature lots of research has been carried out on $\mathrm{NoC}$ implementation for specific SoC architectures. For NoC implementation targeting general purpose applications, with its deadlock free nature and its low logic requirement, it has been observed that wormhole switching technique was the preferred choice as compared to store and forward and virtual cut-through switching [51]. A low area implementation of wormhole switching was given in [52] for 2D mesh topology and very promising results were reported both in terms of area requirement, bandwidth and latency.

In addition to $\mathrm{SoC}$ design, the $\mathrm{NoC}$ concept was studied for reconfigurable architectures also. In [53], Majer et al. presented a dynamically changing network architecture, called DyNoC, for run time partially reconfigurable architectures. In DyNoc, similar to our model, the reconfigurable device is partitioned into 2D small sized grids and area between the grids is used for NoC implementation. The user applications were placed onto this fine grained grid, where the allocated region must be in rectangle shape. When an application was placed, the reconfigurable device resources allocated for $\mathrm{NoC}$ on the placed region were also used for user application. Therefore a dynamic network architecture was proposed to cope with changing network topology and three different routing techniques were discussed.

Another dynamic network architecture for run time reconfigurable architecture was studied in [54]. The proposal was to place a network switch while placing a user application on to device. The placed switch was connected to the rest of the network by using the nearest available switch by reconfiguring necessary wires between two switches. Routing decisions are kept on a dynamic table which was updated when placement or removal of a user application takes place. Whenever an application completes its operation, the associated switch was removed 
from the reconfigurable device surface also.

Corbetta et al. [55] has proposed a communication infrastructure for reconfigurable system implementation. This infrastructure aimed to reduce the design complexity of communication cores and provide fault tolerant communication support. In spite of these advantages, large resource requirement and long reconfiguration times were reported.

In 2009, a dynamic scalable communication structure for dynamically reconfigurable FPGAs was proposed in [56]. With the aim of providing communication between dynamically placed modules the proposed method, called as $\mathrm{CuNoC}$, routes the addressed data packets from source to its destination over a dynamically changing connectivity. Similar to DyNoc, the surface was first partitioned into processing elements and communication unit. A number of processing element and communication unit resources were used for task implementation and it was required that each task has at least one communication unit. With similar communication bandwidth, the authors reported very low logic requirement for the network port as compared to DyNoc.

To the best our knowledge, none of the existing NoC implementations is suitable to be directly used in our computing platform. Although dynamic behaviors are handled in some of the discussed studies, the communication requirements of our platform are completely different than the reported ones. None of the above implementation gives a solution for external memory communication or intra-task data path communication. A dynamically changing network requires too much time adopt itself to changing reconfigurable hardware usage. Since we expect very frequent changes in reconfigurable hardware surface usage, such a network will also not be useful for our computing platform.

\subsection{Inter Block Communication Network Architecture}

The simplest way to implement the IBCN is to use a shared bus architecture as given in $[19,17]$. However, due to limited bandwidth and implementation difficulty in our 2D reconfigurable hardware surface partitioning, we prefer to employ a switching NoC architecture similar to SoC architectures. The proposed NoC implementation is specifically designed and developed for our reconfigurable platform to meet the traffic demand discussed above in Section 5.1. The architectural details of our NoC implementation for IBCN is given below. 


\subsubsection{IBCN Topology}

The NoC topology is a key factor for the IBCN implementation. Since part of the reconfigurable hardware is allocated for IBCN implementation, the IBCN topology must be chosen in a such a way to minimize the logic block requirement and to provide a high throughput while being scalable with the number of user blocks. In literature a number of NoC topologies were discussed for 2D grid based placement of IPs.

In the Octagon topology [57], every node in the eight node network was connected to three other nodes such that any two nodes in the network are at most two hops away. It was possible to expand the network by connecting more octagon topologies with each other. With such a connectivity, it was possible to provide high bandwidth with low latency for random source destination pair. Despite these advantages, the Octagon topology requires too much area for its implementation. Indeed, this area requirement grows exponentially with increasing number of nodes.

Pande et al. [58] has proposed a butterfly fat tree NoC topology, where four IP blocks are located in leaves connected to switches and a switch was connected to two parent switches. In this manner a scalable NoC topology was achieved with very low communication latency between nodes having the same parent node. When traffic generation becomes random, as the order of parent nodes increases, i.e. the subnet size grows, latency increases. In order to get rid of this limitation, a fat-tree network topology, called SPIN, having two stage parent switch was used [59]. In SPIN topology, a switch was connected to four parent switches to minimize the hop distance and to provide alternative paths. Although, low latency and high bandwidth was achieved, SPIN topology suffers from large area requirement.

Since our placement strategy can place the context of two user blocks having data path between them onto any free location on the reconfigurable hardware surface, the traffic demand in our computing platform can also be modeled as random. Therefore, we preferred to use 2D mesh architecture because of its simplicity, less logic resource requirement and suitability for our partitioning in terms of placement, routing and scalability.

Our proposed IBCN topology is based on a 5-way switch, where one port of the switch is connected to the user block and the remaining four ports are connected to 5-way neighboring switches in north, west, south and east directions. Although there is a single SAC, which 
is responsible for host and memory access, we introduce multiple access points (APs) at IBCN and SAC junctions. With the existence of these multiple IBCN APs, the traffic demand towards host and memory is routed to the nearest AP resulting in better IBCN throughput and latency compared to having a single access point to SAC. The APs communicate with the SAC in a round robin fashion for fair access to external memory and reconfigurable hardware operating system circuitry. Figure 5.1 illustrates the proposed IBCN topology for $3 \times 3$ surface partitioning.

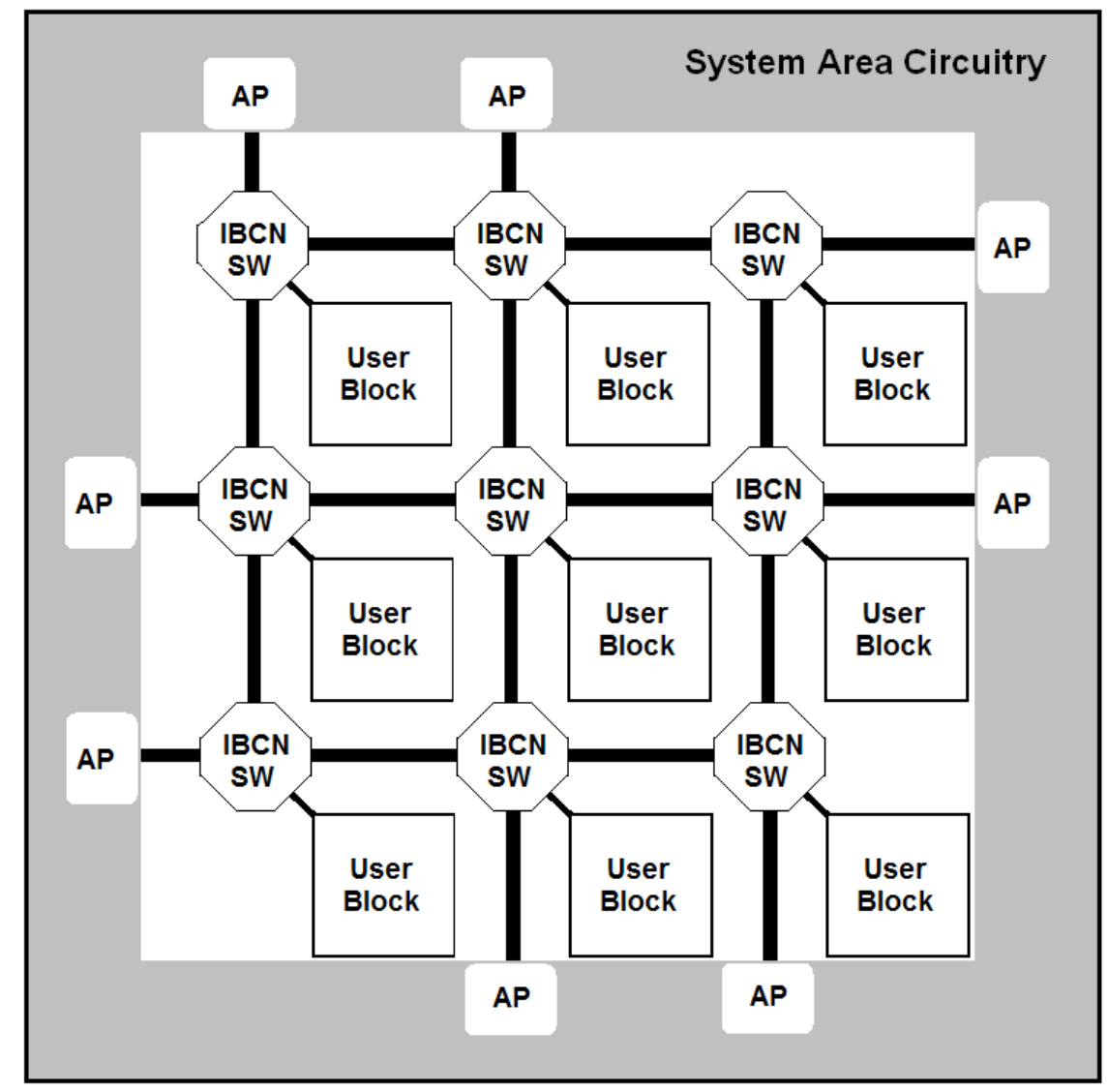

Figure 5.1: IBCN topology for $3 \times 3$ surface partitioning

\subsubsection{IBCN Switching and Flow Control}

In the literature both circuit and packet switching techniques are employed in NoC architectures. In our case however, circuit switching technique is not applicable to IBCN due to 
random traffic demand and short packet sizes. Therefore, a packet switching technique is employed in the proposed IBCN architecture.

Store\&forward, virtual cut-through and wormhole techniques are among the existing packet switching methods. All of these require buffers at the switches for temporary storage of the network packets. In store\&forward mode, a switch waits until a packet is completely received and after reception of the packet contents are examined to decide what to do, which implies per-switch latency. Therefore its overall latency is directly related to both packet size and hop distance between communicating pairs. On the other hand, in virtual cut-through mode, a switch can forward a packet as soon as the next switch gives a guarantee that a packet will be accepted completely without waiting to receive the whole packet. But, it is still necessary to have a buffer to store a complete packet, like in store\&forward mode, but with lower latency communication in this case.

Both store\&forward and virtual cut-through modes require large buffer space especially for large packet sizes. In order to decrease this buffer space requirement, a variant of virtual cutthrough mode called wormhole switching [51] is used. In this mode, a packet is composed of fixed size units called flits (flow control digits-the smallest unit of flow control). The header flit reserves a path from source-to-destination while it is routed. Rest of the flits follow this path in a pipelined manner. Finally, the tail flit removes the reservation on the path.

Due to its low buffer space requirement and low latency, we prefer to use wormhole switching mode. Despite these advantages, this method causes path blocking between source and destination if buffer space is kept low and packet size is large. One remedy to overcome this limitation is to route the traffic, which have large packet size, to the nearest AP for host and memory access. The other large sized packet flow is due to data path communication in our IBCN and we try to minimize path blocking due to such packets by our mapping technique, which will be discussed in the next chapter.

\subsubsection{IBCN Routing}

In addition to path blocking problem, the wormhole routing comes with a need for routing path selection. In wormhole routing, a deadlock occurs if two messages wait for reserved resources in a cyclic manner. Deadlock problem can be solved by breaking the circular dependencies 
among packets. A simple solution is to use dimension-ordered routing where packets always use one dimension first, e.g., column first, upon reaching the destination row (or column), and then switching to the other dimension until reaching the destination. Since packets always use the same path for the same source-destination pair, dimension-ordered routing is deterministic and it cannot avoid contention. Whenever contention occurs, packets have to wait for the channel to be free.

Another other way to solve deadlocks is to use virtual channels; where a physical channel is split into a number of virtual channels. Although this method requires large buffer space, deadlocks can be solved and performance can increase, because of having packets using virtual channels to get rid of contention.

From the above discussion; since we will implement IBCN using reconfigurable device resources, it is obvious that the most area efficient solution is to use dimension-ordered routing to avoid deadlocks. However, dimension-ordered routing is applicable only to the traffic between user blocks, which is the data path communication. Since traffic involving SAC is routed to the nearest AP, this selection may not obey the dimension-ordered routing and it is still possible to have deadlocks. In order to solve this deadlock problem and provide a better bandwidth, two virtual channels are used in our IBCN architecture. In the first channel, traffic between user blocks are routed with dimension-ordered routing in XY manner. The other virtual channel is used for traffic between user blocks and SAC with nearest AP routing policy.

\subsubsection{IBCN Switch Structure}

The IBCN switch is a five-way switch with two virtual channels using wormhole switching mode. As depicted in Figure 5.2, the IBCN switch is composed of four input buffers operating in FIFO mode and associated control logic. Since the fifth port is connected to user block, the buffer for this port is implemented in the user block and is a part of hardware task circuitry.

In conventional NoC architectures, switches are connected to each other via on chip copper wires as shown in Figure 5.3(a) and these copper wires are generally implemented on outer copper layer and can be cross routed over nodes. In contrast to conventional NoC architectures, we use a dedicated area in reconfigurable surface for our IBCN and do not allowed 


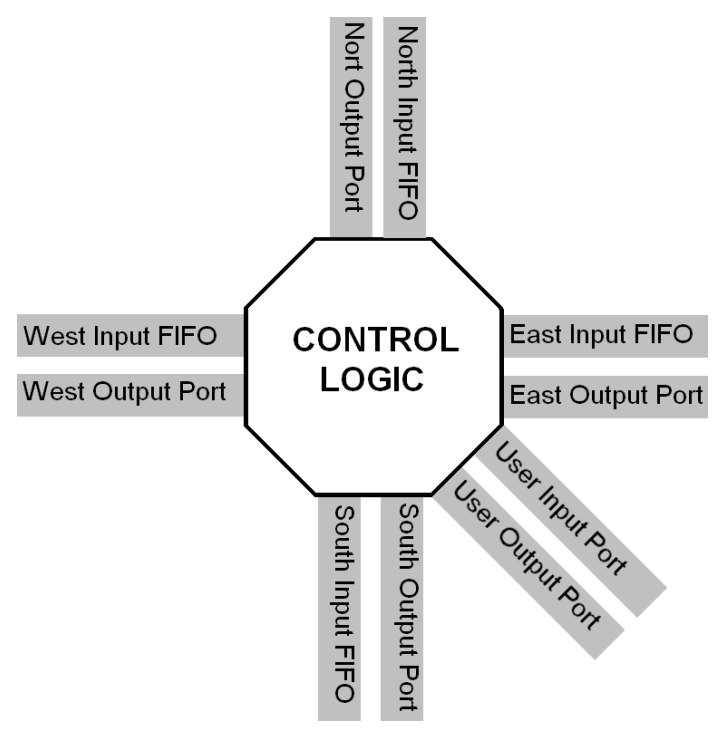

Figure 5.2: IBCN switch structure

wires over user blocks because IBCN should be functional during reconfiguration of the user blocks. In order not to waste the area between two switches for wired connectivity, this area is used for additional buffer implementation and neighboring IBCN switches are connected to each other via these buffers, as shown in Figure 5.3(b).

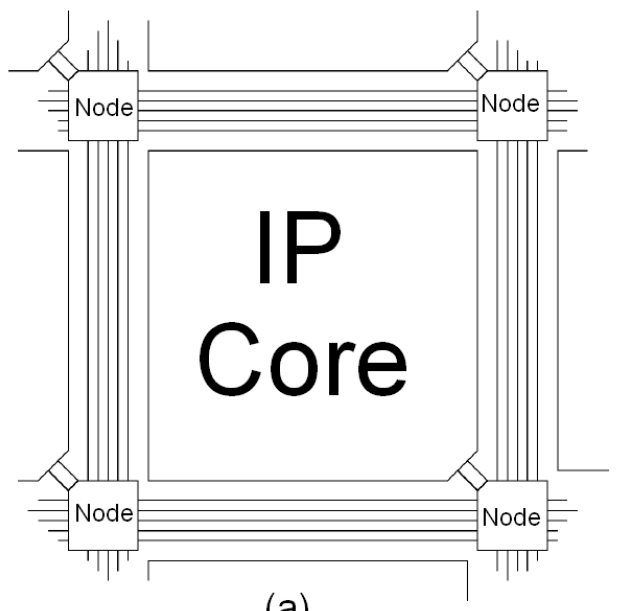

(a)

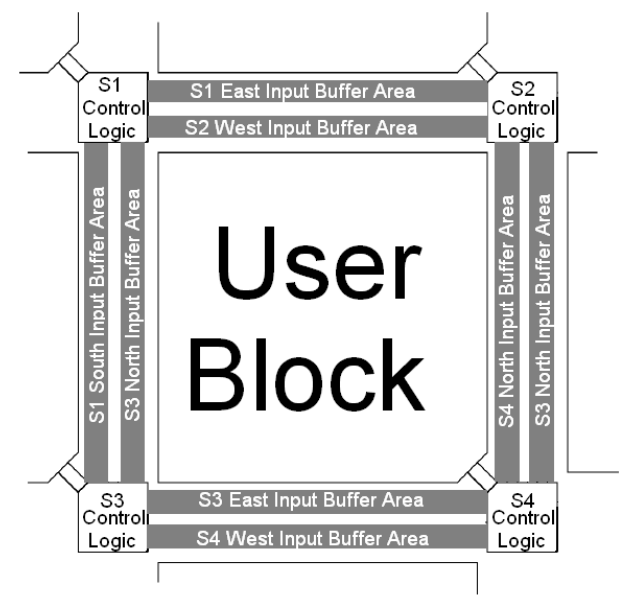

(b)

Figure 5.3: Physical location of IBCN switch buffers

The buffers between switch control logic are dual port FIFOs where one of the control logic pushes data into FIFO as long as there is room for new data and the other one pops data out 
of FIFO till FIFO becomes empty. The link between two switches becomes stalled whenever the FIFO is full. As was mentioned earlier, two virtual channels exists in IBCN switch. Each channel requires its own buffer space and control logic, which shares the connection between switches among these virtual channels. Since a FIFO based buffer is used for both storage and connection purpose this buffer must provide two FIFO functionality. One simple way to implement this functionality is to employ two separate FIFO and a multiplexer to select the requested FIFO as shown in Figure-5.4. The control logic pops data from input virtual channel FIFO and push it to output virtual channel if;

- there is data in corresponding virtual channel input FIFO

- there is room in corresponding virtual channel output FIFO

- the time slot is allocated to corresponding virtual channel.

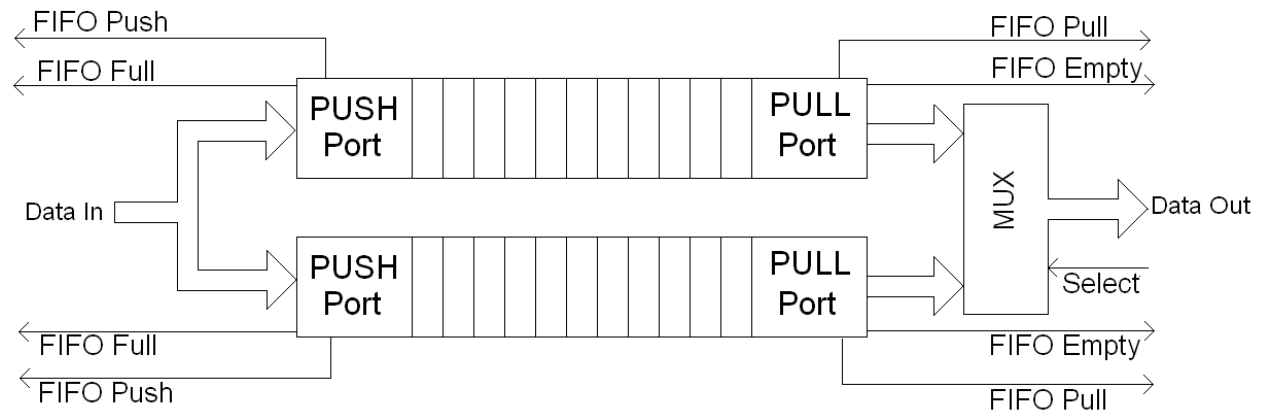

Figure 5.4: Two virtual channel based buffer architecture in IBCN

One-directional nearest AP routing is used for SAC based traffic, where the direction is based on the position of the user grid communicating with the SAC. Therefore the virtual channels are implemented either in horizontal or vertical directions only. Hence out of four ports, at most two ports have virtual channels. The layout of IBCN control logic and virtual channel FIFOs for a $5 \times 5$ surface partitioning is illustrated in Figure 5.5 as an example.

The control logic of the IBCN switch is responsible for reading data from the input buffer (pop from FIFO) and writing to the addressed output buffer (push to FIFO). In addition, the time slot multiplexing for virtual channel FIFO access is handled by this control logic. As depicted in Figure 5.6, the control logic is composed of five $4 \times 1$ multiplexers, buffer control logic to 


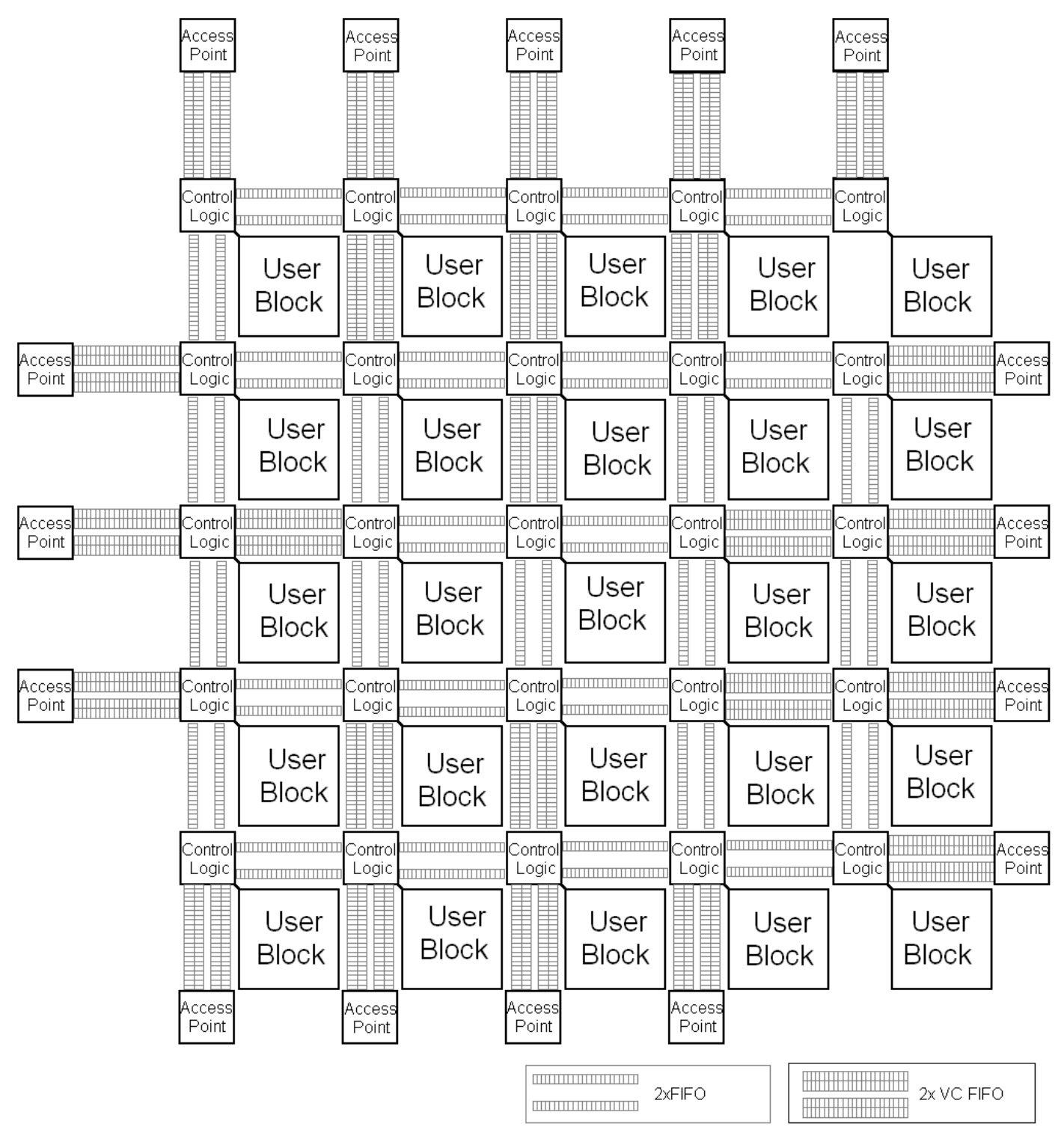

Figure 5.5: An example 5x5 IBCN topology with FIFOs 
generate FIFO push and pop signals and a switching table to hold the current connection within the switch.

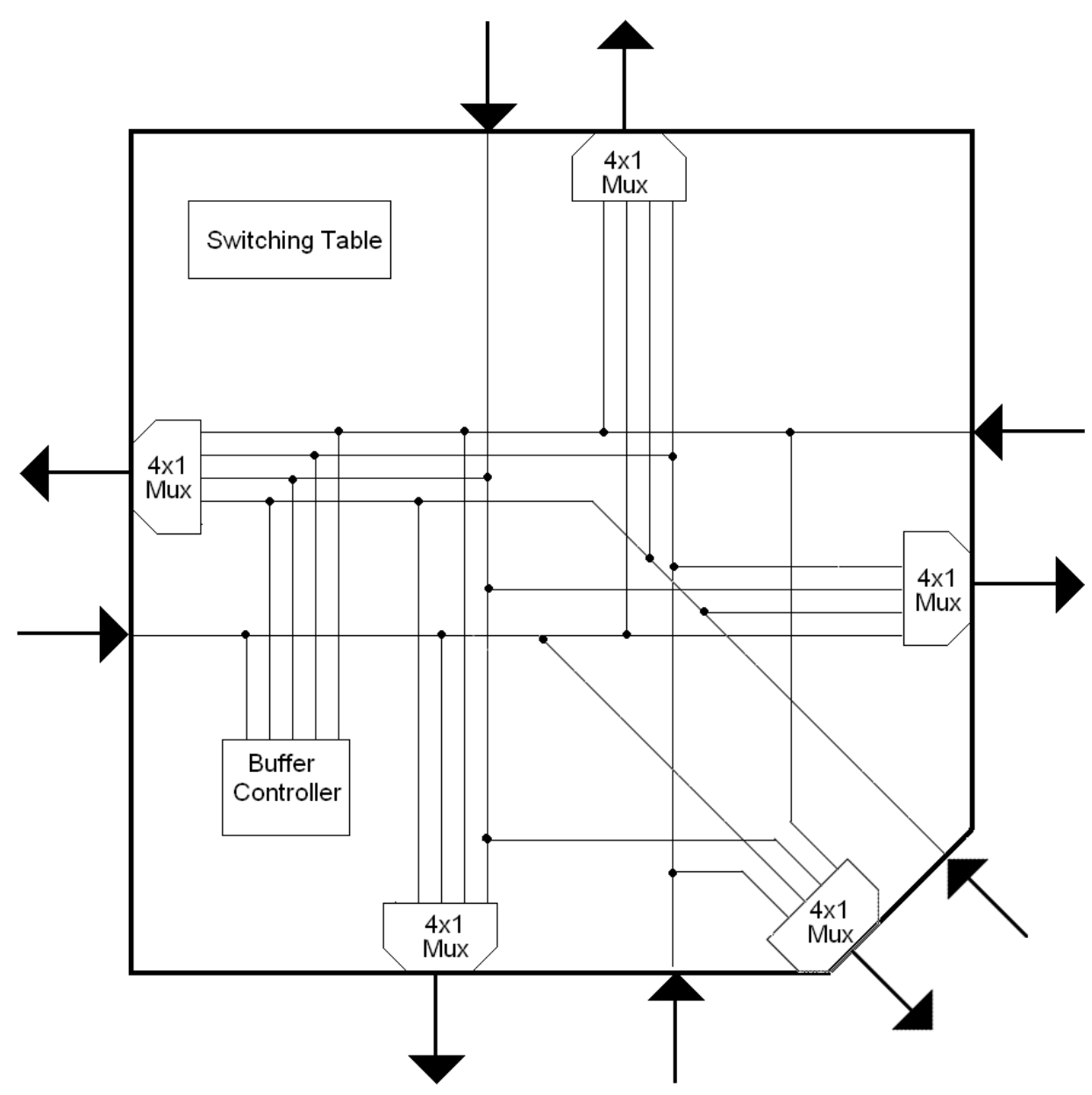

Figure 5.6: IBCN Switch control logic architecture

Our implementation of the switching table, which supports virtual channels, is a modification of the HERMES switch [52]. Using this table, the buffer controller logic generates the necessary control signals to read from an input buffer and to write to an output buffer, as long as there is data in input buffer and room in the output buffer. Upon receiving the header flit; if the corresponding output port is free, the table is updated. Otherwise the header flits wait in FIFO till the output port becomes free. Upon receiving the last flit, the corresponding output port free flag is set. The input channel assignment to an output channel is done in a round robin manner. Path arbitration among virtual channels is also done using round robin arbitration. 
Figure 5.7 illustrates an example channel allocation and associated switching table for a case with two virtual channels in horizontal direction. Table entries show, for each port, whether the port is active as an input or an output port (port free flag) and its associated input/output port numbers.

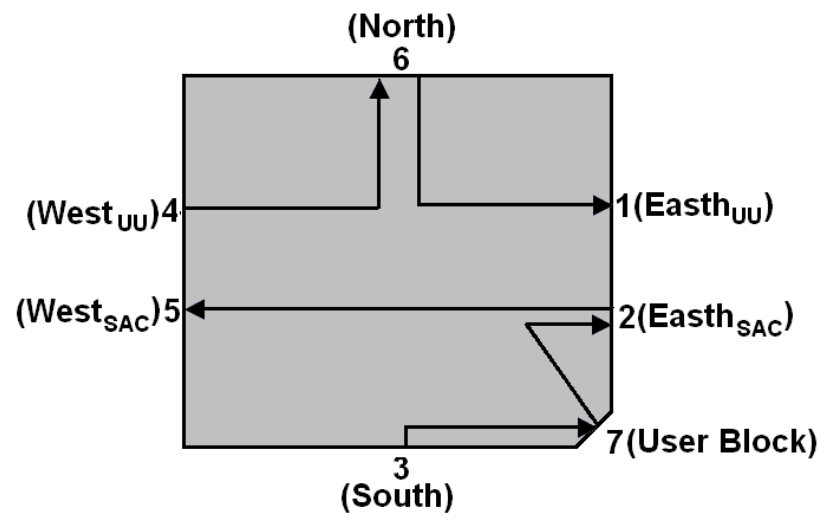

\begin{tabular}{|c|ccccccc|}
\hline & 1 & 2 & 3 & $\mathbf{4}$ & $\mathbf{5}$ & 6 & 7 \\
\hline In Free & 0 & 0 & 1 & 1 & 0 & 0 & 0 \\
\hline Out Free & 1 & 0 & 0 & 0 & 1 & 0 & 0 \\
\hline In Port & 6 & 7 & - & - & 2 & 4 & 3 \\
\hline Out Port & - & 5 & 7 & 6 & - & 1 & 2 \\
\hline
\end{tabular}

Figure 5.7: A switching table for a switch having virtual channels in horizontal direction Note that Port $_{U U}$ represents the virtual channel for user block to user block traffic and Port $_{S A C}$ represents the virtual channel for traffic involving SAC.

\subsubsection{IBCN Packet Format}

In IBCN, payload size $P_{S}$ for a flit is a user defined parameter and we add an extra two bits $F_{0}, F_{1}$ for flit type coding (see Figure 5.8). The encoding of these two bits is given in Table 5.2. Payload size $P_{S}$ should satisfy $2^{P_{S}} \geq(m \times n)$ constraint, where $(m \times n)$ is the number of user blocks in reconfigurable hardware surface. This is because the header flit must include all the information about the destination. For a header flit the payload is the destination user block number for data path communication between two user blocks, and traffic type for other flit types involving SAC. When destination is SAC, the second flit is used to specify source user block number for memory read request, FINISH SIGNAL or SYS_CAL request. When the source is SAC, this flit is the destination user block number for memory read response, START SIGNAL or TASK_CALL. 


\begin{tabular}{|l|l|l|}
\hline $\mathrm{F}_{0}$ & $\mathrm{~F}_{1}$ & Payload $\left(\mathrm{P}_{\mathrm{s}}\right.$ bit $)$ \\
\hline
\end{tabular}

Figure 5.8: IBCN flit format

Table 5.2: IBCN flit type encoding

\begin{tabular}{ll}
\hline$F_{0} F_{1}$ & Flit Type \\
\hline 00 & Payload Flit \\
01 & Tail Flit \\
10 & User Block to User Block Traffic Header Flit \\
11 & SAC to User Block Traffic Header Flit \\
\hline
\end{tabular}




\section{CHAPTER 6}

\section{HARDWARE TASK PLACEMENT AND MAPPING PROBLEM}

As was mentioned in Chapter 3, it is possible to partition a hardware task into a set of execution blocks working in parallel and having data dependency. Although it is not recommended to partition part of circuitry that requires a high bandwidth data dependency into different execution blocks, such a partitioning may be inevitable in some applications due to large circuit size or different clock frequency requirements. In case of such a high bandwidth data path communication requirement, the execution time can also increase if the communicating pairs are placed far from each other on the reconfigurable hardware surface. In addition, due to path blocking nature of wormhole routing, the execution time can increase further if parts of the communication path is used by other communicating pairs also.

Regardless of the bandwidth requirement of the communication path between execution blocks working in parallel and having data dependency, the execution time of a hardware task can increase if user blocks are not placed/mapped onto the reconfigurable hardware surface properly. Since our IBCN implementation provides (see Chapter 8) a huge bandwidth and deep buffers to decrease path blocking probability, this increase in execution time will be negligible if the hardware task partitioning is done appropriately. However, there is still a need for an efficient mapping technique for better system performance and to support high bandwidth data dependency partitioning.

Similarly, the communication latency of the traffic between user blocks and SAC has negative effect on hardware task execution time. Since the memory and reconfigurable hardware operating system have a fixed bandwidth, overall system performance does not depend on the physical mapping of the hardware task user blocks. However, because of the round robin mechanism used for servicing AP requests, some user blocks can have shorter SAC access 
latency if the load on APs are not balanced. Therefore, a mapping technique is still required to provide fair SAC access to hardware tasks. This fairness becomes critical especially for hard real time systems requiring a deterministic response time.

From the above discussion, it is obvious that an efficient mapping technique is needed to minimize the communication latency and to provide fair SAC access. In the following sections, a brief literature survey is given on the related work. Then the problem definition is given with performance figure of merits to be met by our mapping algorithm. A problem analysis is then given followed by an ad-hoc algorithm proposal for mapping. Finally, the performance of the proposed mapping technique is evaluated.

\subsection{Literature Survey on Mapping Problem}

The mapping problem in this thesis work is very similar to that of SoC designs. As the number of cores in SoC architectures grows rapidly, efficient networking and mapping algorithms are needed. The aims of mapping in SoC architectures are selection of suitable computing resources, routing of communication between these computing resources and allocation of network channel capacity over time. For SoC architectures, the mapping problem is a well studied field in the literature and some of the major works are briefly discussed here.

The earliest work in this field was the mapping of IPs, such as GPP, DSP and memory subsystems, onto a generic regular NoC architecture to minimize total communication energy while satisfying bandwidth allocation constraints and constructing deadlock free routing [60]. The authors proposed a routing scheme with an energy model to formulate the routing problem and a branch-and-bound algorithm was used to solve the mapping problem. An improvement to this branch-bound algorithm with experimental study results were also reported in [61].

Similarly, Lei and Kumar [62] studied the static mapping of a task graph composed of IP vertices and communication channel edges onto a mesh NoC architecture with IP nodes. In order to minimize the communication delay and hence the execution time, a two level genetic algorithm has been developed for vertex mapping problem. For this mapping problem a special delay model was given to represent the communication delay between two nodes with respect to their physical locations. 
For mesh NoC architectures with high bandwidth requiring IP cores, using a single path routing approach places these high bandwidth requirements directly on each link. In [63], it was proposed that the bandwidth requirements can be reduced by splitting the traffic into multiple paths and a static mapping technique was proposed to satisfy the bandwidth constrains of the IP cores. The proposed technique, called NMAP, tries to minimize the average communication delay and it has been evaluated both for single path and multi-path cases. The proposed mapping algorithm starts with an initial assignment of IP cores and improves the solution by iteratively solving the shortest path problem for each core pair to minimize the delay. The authors reported their algorithm's performance for a number of video applications.

Existing methods discussed up to here, either try to minimize the energy or communication delay. These methods are static and do not consider dynamic effects such as delay variation due to switch input buffer, which in fact has a great impact on mapping. In [64], it has been shown that optimization of just a single parameter may result in unacceptable solution when the other parameter was not considered. In order to solve this problem and take dynamic behaviors into account, a multi-objective mapping environment was discussed and a genetic algorithm based method was proposed.

A similar multi-objective study has been carried out Srinivasan and Chatha [65] to minimize the total communication energy while satisfying the bandwidth constraints on router and latency constrains on traces for a mesh NoC. It was observed that the communication energy consumption is directly related to the congestion rate and hence the proposed mapping technique tries to minimize congestion. The proposed mapping technique, called MOCA, was a two phase method where in the first phase energy versus latency trade-off was done for mapping and in the second phase a custom route was found to minimize energy and to meet latency constraint. A comparative study against NMAP [63] has been carried and it has been reported that in most of the cases NMAP fails to satisfy the latency constraints while MOCA meets them.

Most of the mapping techniques are multiphase methods but a unified single phase mapping method, called UMARS, was proposed in [66]. UMARS couples path selection, mapping of cores and TDMA time-slot allocation tasks in order to both minimize the energy and area needed for NoC while satisfying the latency requirement. Since one of the outputs of UMARS is the network itself, this technique was only applicable to very well defined static SoC im- 
plementations.

Up to now, the existing methods mentioned here was performing static mapping of tasks onto mesh NoCs. In [67], Nollet et al. proposed a centralized mapping technique that performs a runtime resource management in a tile based reconfigurable hardware. The proposed method performs run-time task migration by relocating executing tasks onto another tile to meet the changes in user requirements.

Another run-time mapping technique proposed to meet the changes in user requirements was discussed in [68]. In the proposed resource allocation process, user behavior information was used to both give response to real time changes and to minimize the energy consumption and network contention. They have modeled applications as a set of computing resources and proposed their mapping technique for multiprocessing applications. Two mapping algorithms have been proposed that considers both internal and external contention. The authors extended their work in [69].

Although there exists very powerful techniques for mapping applications onto mesh based $\mathrm{NoC}$, which is a similar architecture to our surface partitioning and IBCN, most of them are suitable for static mapping of a number of applications before their field operation. Therefore these techniques are not directly applicable to mapping problem in our reconfigurable computing platform, which requires a run time mapping of user blocks of tasks onto our reconfigurable hardware surface. In a similar way, although some of the existing methods $[67,68,69]$ considers run time changes in user behavior they are still not useful for our mapping requirements because they just try to remap already mapped applications to meet the changes. Therefore, we need a special mapping technique for our reconfigurable computing platform that will try to minimize the effect of IBCN communication on the execution time of user tasks.

\subsection{Traffic Modeling}

As was mentioned earlier, the execution blocks in our methodology are packed into user blocks for efficient reconfigurable hardware utilization. During this packing process, if the data dependent execution blocks are packed into the same user block, simply the Data Out port of the execution block generating data is connected to Data In port of the execution block 
processing this data. However, if they are packed onto different user blocks then the Data In ports are connected to IBCN switch User Input Port and Data Out ports are connected to IBCN switch User Output Port. Since a unified memory architecture is used in our computing platform, memory write and read requests of execution block packed into the same user block are aggregated onto IBCN switch User Output Port and User Input Port, respectively. Similarly the traffic between the execution blocks and the hardware operating system is also routed to these ports. With such connectivity, the generated traffic demand of a user block $i$ is modeled with a 3-tuple as, $T U B_{i}\left(M_{W r i t e}, M_{\text {Read }}, S_{D O U T}\right)$ where;

- $M_{\text {Write }}$ is the sum of memory write type traffic demand of the execution blocks forming user block $i$

- $M_{\text {Read }}$ is the sum of memory read type traffic demands of the execution blocks forming user block $i$

- $S_{D O U T}$ is the set of traffic demand where $S_{D O U T_{i j}}$ represent the traffic demand from user block $i$ towards user block $j$, where user blocks $i$ and $j$ are at the same level on the reduced hardware task graph.

With such a constant rate assumption for the user block traffic generation, the traffic demand on an access point $i$ can be modeled with the ordered pair as $T A P_{i}\left(S A C_{\text {Write }}, S A C_{\text {Read }}\right)$ where;

- $S A C_{W r i t e}$ is the sum of memory write type demand of the user blocks accessing SAC over access point $i$.

- $S A C_{\text {Read }}$ is the sum of memory read type demand of the user blocks accessing SAC over access point $i$

Although the traffic between hardware operating system circuitry and user blocks passes also over the access points, this traffic is not taken into account due to its low bandwidth requirement as compared to memory access traffic. 


\subsection{Problem Definition}

During the execution of a hardware task, the context of the user blocks belonging to the same level in the reduced task dependency graph are loaded onto our reconfigurable hardware whenever it is scheduled by the hardware operating system and whenever there are enough free user blocks on the reconfigurable hardware surface. The decision of selecting the physical locations of a user block to load its context is critical and has an impact on overall system performance. As an example, consider the case where the user blocks and the traffic demands are shown as in Figure 6.1 and the corresponding reduced task dependency graph level is to be loaded onto the reconfigurable hardware surface with usage and access point (AP) traffic load as depicted in Figure 6.2. Below, the effect of user block mapping on system performance is discussed for this scenario.
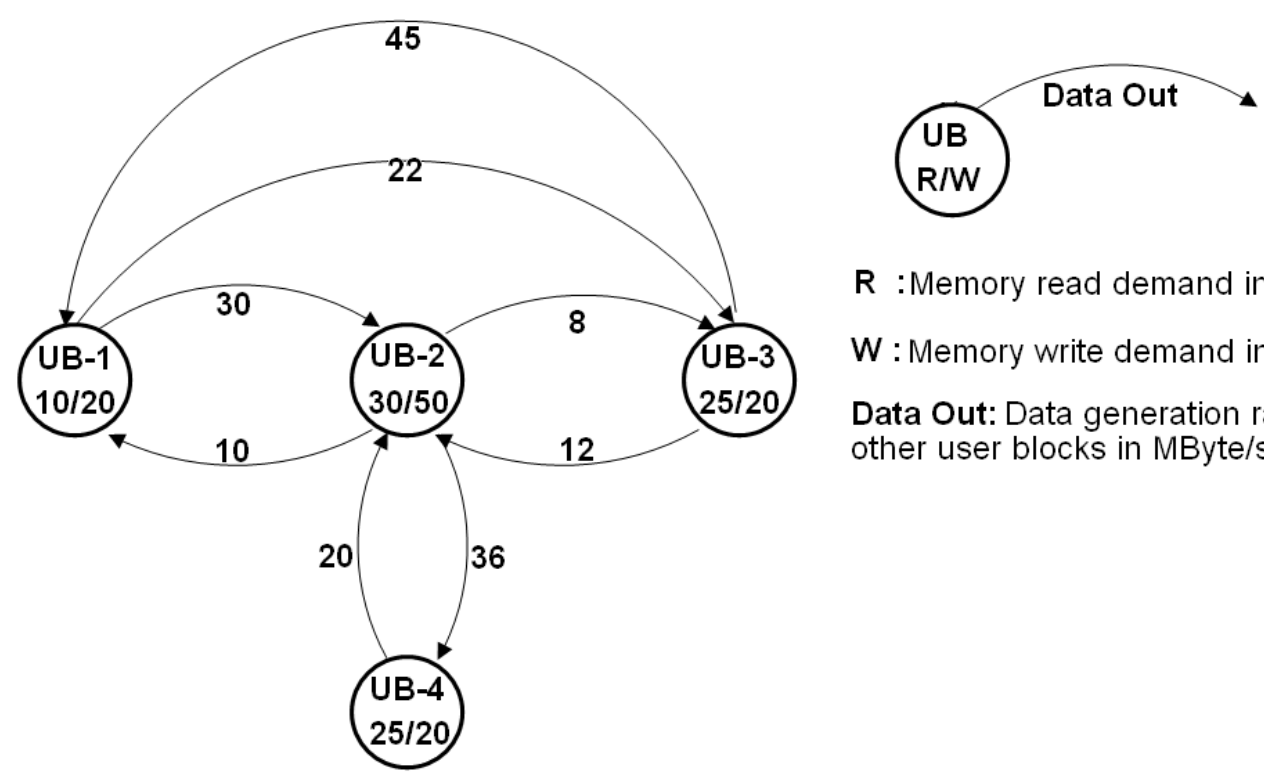

$\mathbf{R}$ :Memory read demand in MByte/s

W : Memory write demand in MByte/s

Data Out: Data generation rate for other user blocks in MByte/s

The nodes represent memory traffic demand (read/write) and edges represent the unidirectional data generation rate between user blocks in MByte/s.

Figure 6.1: An example traffic demand for user blocks of a reduced task graph level

As was mentioned earlier, the data path communication between user blocks have a latency due to packet switching nature and this latency leads to an increase in execution time. Therefore, mapping of data dependent user blocks should be done in such a way to minimize this communication overhead. This requirement is very similar to internal network contention 


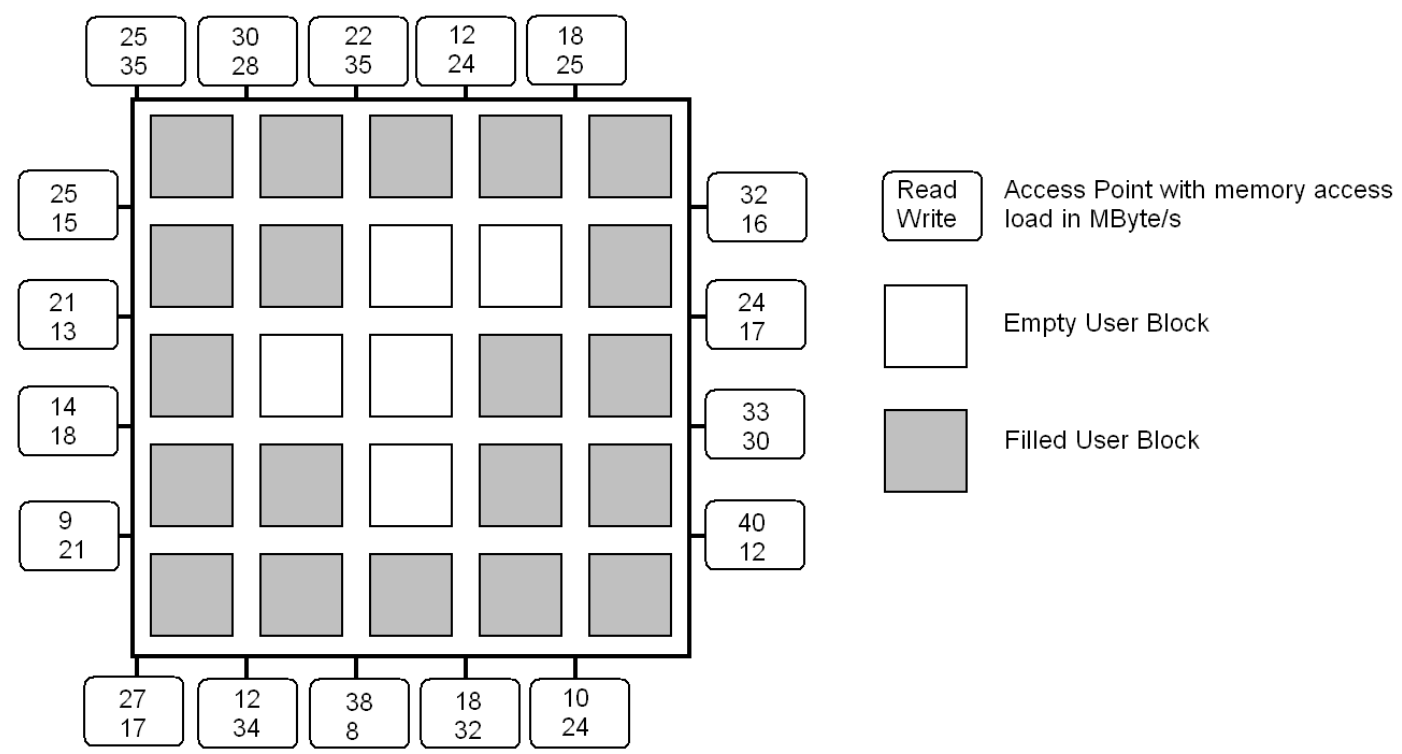

Figure 6.2: An example hardware surface usage and memory traffic load for access points

minimization requirement in existing studies for mapping on NoC architectures. As our IBCN architecture is based on $\mathrm{XY}$ routing with wormhole packet switching, the communication overhead between user blocks can be minimized by minimizing the Manhattan distance. For the above specific example situation, the Manhattan distance and hence the communication overhead is minimized if the user blocks are mapped as shown in Figure 6.3.

Another requirement of the mapping decision is to select the physical location in a way to achieve an efficient memory access. Note that, since IBCN routes memory traffic to nearest access point and access points are serviced in a round robin arbitration manner, the whole memory traffic must be well distributed over the access points. Hence the mapping technique should balance memory traffic over access points also. This load balancing can be achieved by minimizing the standard deviation of each access point load from the average access point load. In Figure 6.4, the optimal mapping to balance access point loads is given for the specific example of Figure 6.1 and Figure 6.2 with updated access point loads. Note that this mapping is different than the mapping depicted in Figure 6.3 which aims to minimize the user block to user block communication overhead only. 


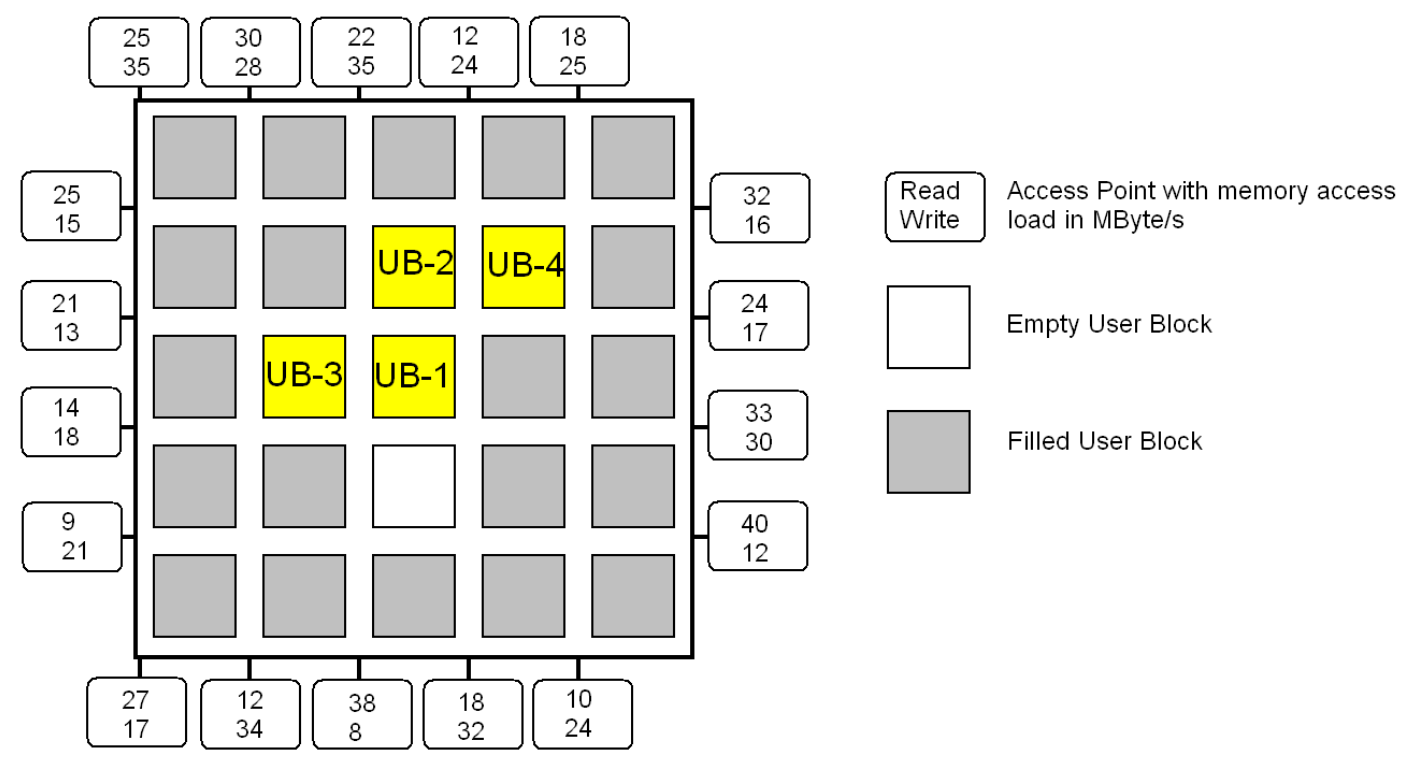

Figure 6.3: Physical mapping of the user blocks onto reconfigurable hardware surface to minimize the Manhattan distance

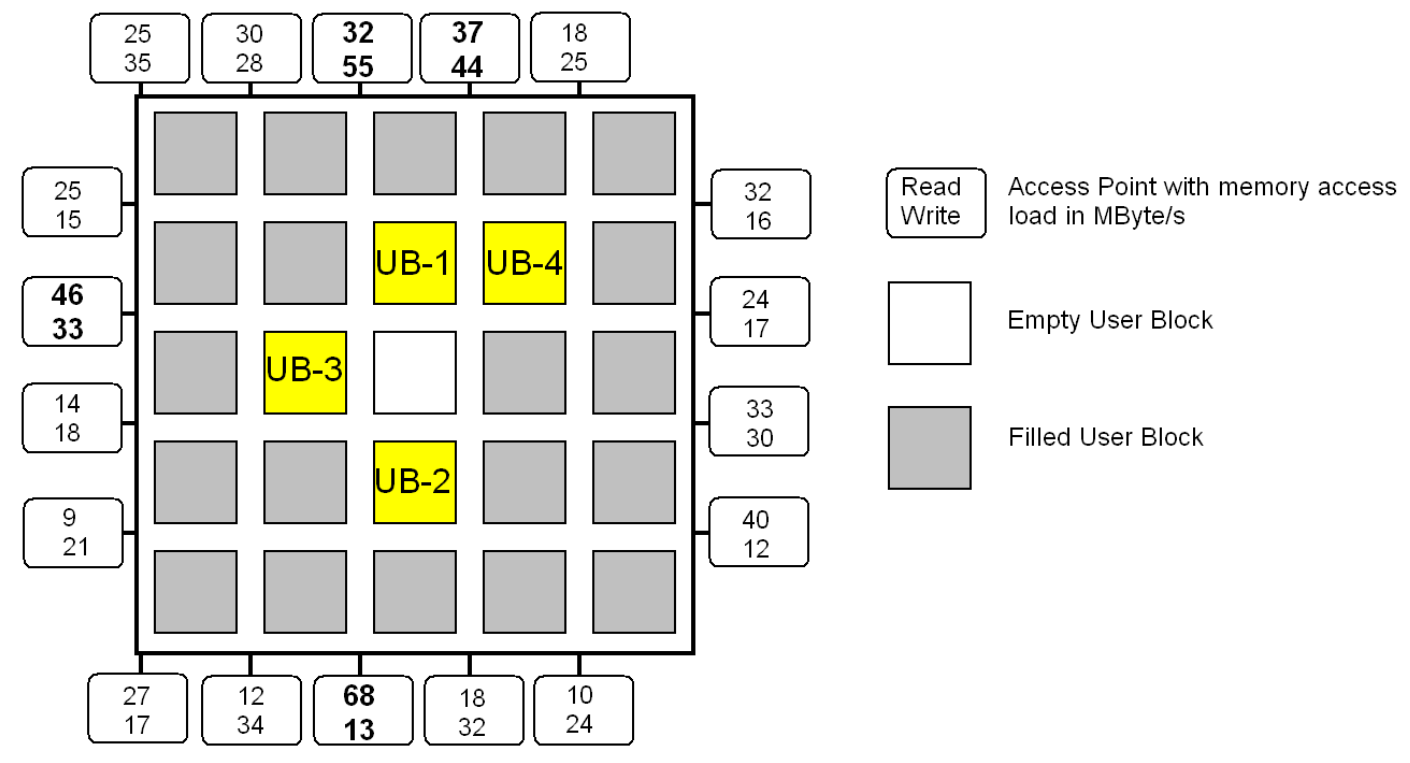

Figure 6.4: Physical mapping of the user blocks onto reconfigurable hardware surface to balance access point loads 
Finally, it is required to map user blocks in such a way that the communication between two user blocks is not affected by the communication of another pair. Due to the nature of wormhole routing, a path from source to destination is blocked till the tail flit is sent by the data generating user block. Therefore, if part of the communication paths of two communicating pairs are shared, one communication must wait till that part of the path becomes free. This waiting time is an additional latency and can be large if large sized packets are employed. In order to minimize this latency due to path blocking, the mapping must be done to minimize the path sharing between communicating pairs. In most of the cases, this path sharing is unavoidable but then the mapping should still be done to form a path such that the sum of the blocking rates of the links of the path is minimized. The path blocking rate can simply be modeled as the sum of the traffic demands on the links of the path. For the specific example described in Figure 6.1 and Figure 6.2, path sharing is minimized by mapping the user blocks as shown in Figure 6.5.

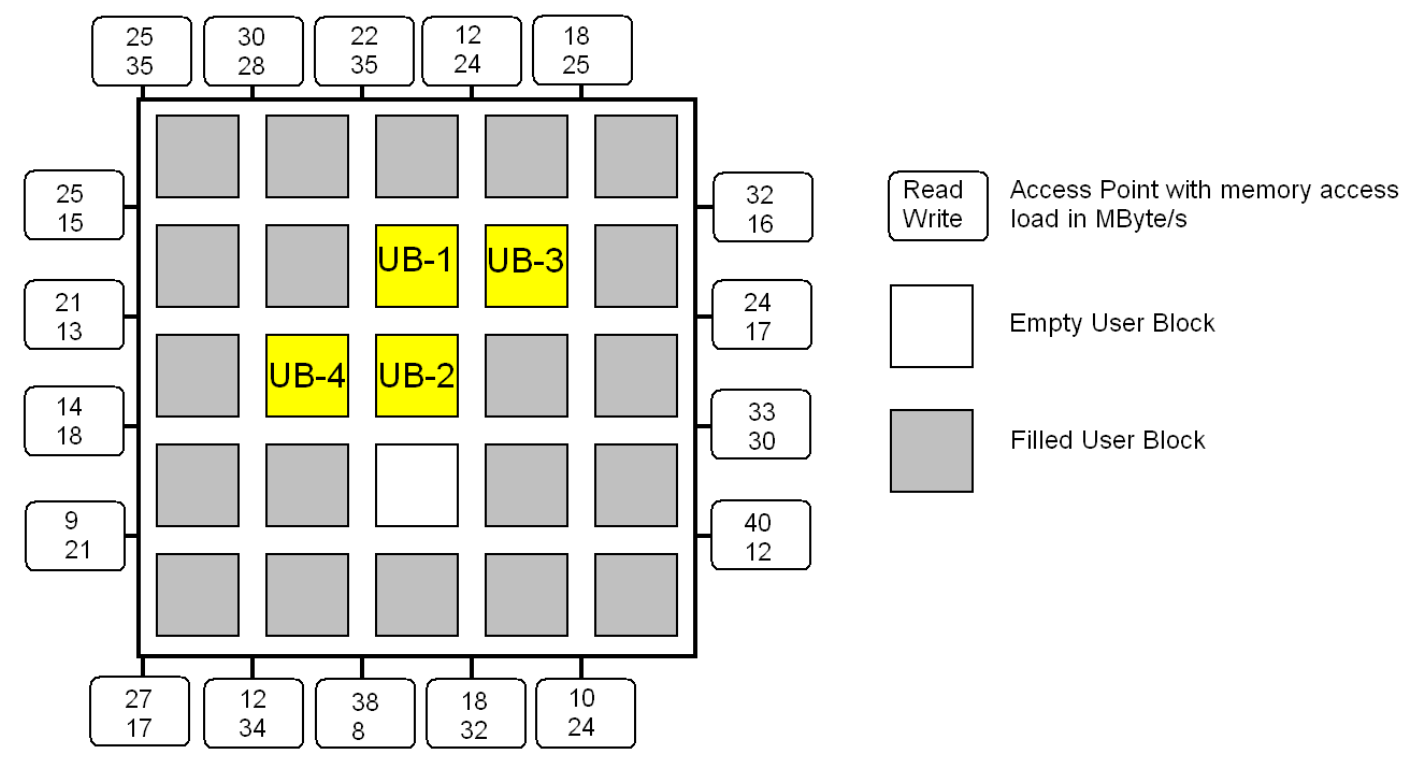

Figure 6.5: Physical mapping of the user blocks onto reconfigurable hardware surface to minimize latency due to path blocking

As shown in the above figures, these figures of merits are satisfied optimally with a different mapping in each case. Therefore there is a need for a multi-objective mapping technique that satisfies these three objectives. Below, formulation of such a multi-objective mapping problem is presented. 


\subsection{Problem Formulation}

Given;

- a reconfigurable hardware with enough free space

- memory traffic demand on access points

- a level of a reduced hardware task graph to be scheduled.

Problem $P_{m}$ :

Find the physical locations of the user blocks that belong to the level to be scheduled.

Such that;

- the sum of Manhattan distances between each communicating user block pairs is minimized

- the standard deviation of access point loads is minimized

- the path blocking rates of the communication paths between user block pairs are minimized

\subsection{Problem Analysis}

Among the three objectives of the mapping problem $P_{m}$, the first objective function to minimize communication latency is a well studied field in the literature and this problem is shown to be NP-complete [61]. Therefore our multi-objective mapping problem is NP-complete too. Hence our mapping technique is designed taking this NP-complete behavior into account.

In addition to the above minimization objectives, our mapping problem has some more additional requirements. The first requirement is for the execution time of the mapping technique. In contrast to existing studies for mapping on SoC architecture, our mapping decision should be given in runtime. Therefore, the selection of physical locations to load the bitstream must 
be given in a very short period of time so that the effect of mapping decision time on the total hardware task execution time is also minimized.

The second requirement of our mapping problem is on the computational resource usage. In our reconfigurable computing platform the hardware operating system is responsible for the scheduling of user blocks as well as the above mentioned mapping decision. In addition, the mapping technique should be designed in such a way that it is suitable for hardware implementation. Since reconfigurable hardware resources are used for hardware operating system circuitry implementation, our mapping technique should also have a low circuit footprint in order to minimize the overhead on device utilization. With these additional requirements and problem characteristic, a mapping technique suitable for hardware implementation with feature aware nature is proposed in the following subsection.

\subsection{Ad-Hoc Mapping Solution}

Before solving the multi-objective mapping problem $P_{m}$, the effect of each objective on the overall system performance is briefly analyzed here. The first objective is to minimize the communication latency due to intermediate store and forward nature of packet switching. This latency has a moderate effect on the user block execution time and hence affects both hardware task execution time and overall system performance moderately.

The second objective is about balancing memory traffic on access points. As was discussed before, unified memory has a fixed bandwidth and this bandwidth is shared between access points and hence between user blocks. This fixed bandwidth is not within the scope of our study and it is assumed that this interface has necessary bandwidth for all user blocks at any time. This balancing definitely has an effect on the execution time of the individual user blocks and hence on the overall hardware task. However, when overall system performance is considered, memory interface bandwidth affects total system performance while access point load balancing having no effect on it. Therefore, the second objective can be considered as the least significant one during the mapping decision.

The third objective is the minimization of the path blocking rates of the communication paths. Depending on the packet size, some links can be blocked for a long time. The result of this blocking is an additional latency on communication resulting in larger execution times for 
hardware tasks and hence degraded overall system performance.

When the degree of effect on the total system performance is considered, we believe that the third objective is the most important factor and should be considered first during the mapping process. The path blocking arises for two reasons. The first one is using a communication path passing through an already used area on the reconfigurable hardware. The second one is path sharing of mapped user blocks. Our proposed mapping technique decouples these two components and handles them separately.

This separation is achieved by a two phase mapping solution. In the first phase, a suitable region is allocated to required number of user blocks without affecting the already executing user blocks. In the second phase, user blocks are mapped on this region such that the Manhattan distance and path blocking rates are minimized while trying to balance the access point loads.

Our technique presented here requires data dependency between user blocks. The reduced hardware task graph level may be composed of two sets of user blocks, one having data dependency between a group of user blocks and the other having no data dependent user blocks. In our mapping technique, each data dependent user block group is handled separately. Our hardware operating system scheduler will be responsible for providing the data dependent user block group of the corresponding hardware task graph level to the mapping hardware circuitry.

\subsubsection{Phase-1: Region Allocation}

In this phase of our mapping technique, part of the reconfigurable hardware surface is allocated for the user blocks having data path communication. The region allocation process is done in such a way that the communication paths of already mapped user blocks are not used. Due to XY routing, such new communication paths can be provided to user blocks if and only if the allocated area is contiguous set of free user blocks and the allocated free user blocks form a rectangular shape. As depicted in Figure 6.6, when non-rectangular shaped areas are used it may be possible to have new communication paths passing over the already mapped user blocks, which will disturb the existing communications also. 


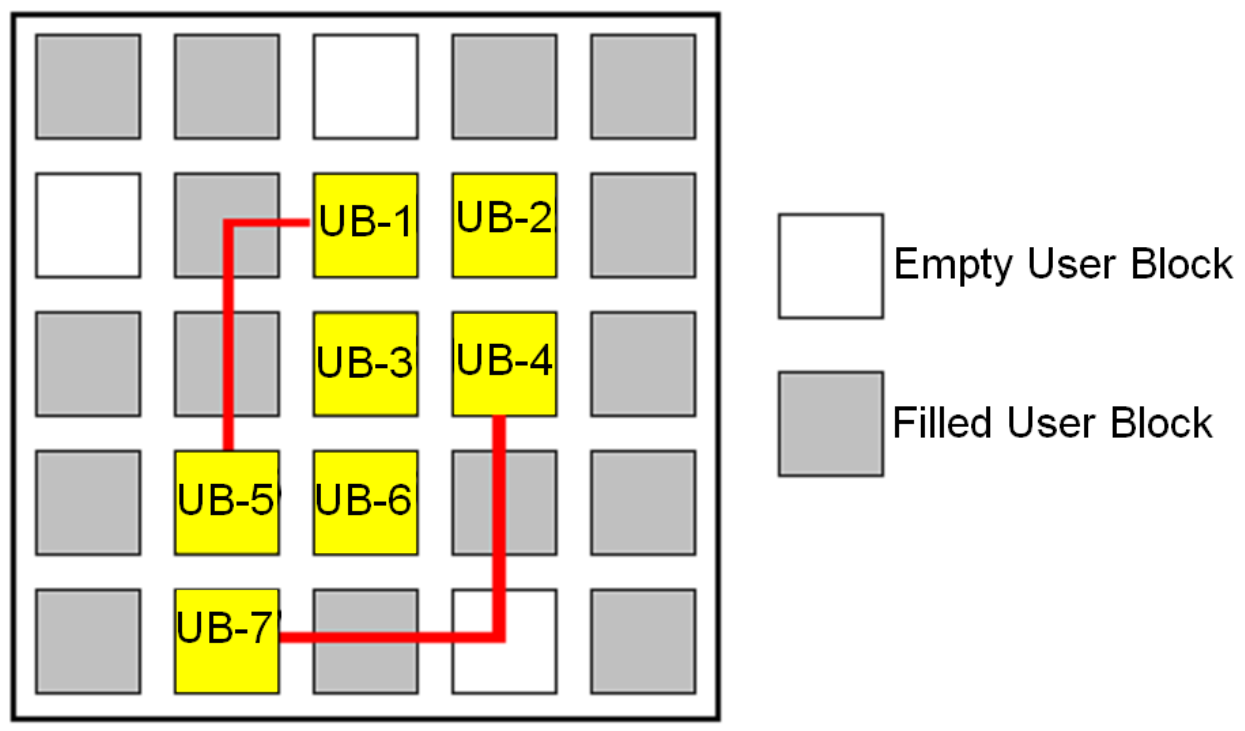

The communication path between UB7-UB4 and UB5-UB1 pass over filled user blocks when nonrectangular shaped mapping is used.

Figure 6.6: A non rectangle shaped mapping disturbing existing communication

The second criterion of region allocation process is to use the free area in a future aware way so that the remaining area can be allocated as a set of user blocks forming a rectangle shape for other user blocks to be mapped. This objective can alternatively be defined as the minimization of the fragmentation on the free area. Minimizing the fragmentation will help mapping user blocks with data path onto a contiguous area so that both the Manhattan distance and path blocking rate can be minimized. It is obvious that allocating free user blocks forming a non-rectangular shape will result in more fragmentation as compared to a rectangular shaped allocation.

The key factor in the allocation of a rectangular shaped region formed by free user blocks is the width and height of the rectangle needed to map the user blocks. A possible solution then is to find the optimum width and height allocation of the rectangular area in our bitstream generation. Since we will obtain the reduced hardware task graph and its levels during our offline execution packing process (to be explained in the next chapter), we can map the user blocks with data dependency to an optimum shaped rectangle so that both the Manhattan distance and path blocking rate can be minimized. With these optimal rectangles formed by user blocks, it is possible to use the existing solutions in the literature (for example [13] and [14]) that places rectangles onto reconfigurable hardware surface in runtime. However, it is obvious 
that with such a region allocation, fragmentation will be high because of selecting rectangle parameters without any information about the current reconfigurable hardware usage.

In order to minimize the fragmentation and hence path blocking rate, we propose a simple run-time region allocation technique that selects a free set of user blocks forming a rectangle whose width and height is determined using information about the current reconfigurable hardware usage. In this technique, a list of free user blocks forming rectangular areas on the reconfigurable hardware surface is kept. This list is a sorted one with the following rules;

Rule-1 Sort with respect to descending rectangle size order.

Rule-2 If there are rectangles with equal size, sort with respect to ascending $\mid$ height - width $\mid$ value

Rule-3 If there are rectangles with equal $\mid$ height-width| value, sort with respect to ascending value of the current summation of the access point load. This summation of access point load is calculated by only considering the access points used by the user blocks forming the particular rectangle area under consideration.

In the above sorting process, Rule- 1 aims to minimize the fragmentation. Rule- 2 is used to choose the rectangle with most square like shape to allow a more flexible mapping so that the Manhattan distance and path blocking rate can be minimized. Finally, Rule-3 is applied to select the region with a lower access point load so that the standard deviation of the access point loads can be minimized.

With this sorted rectangle list in hand, wherever a request to map a set of user blocks is issued by the hardware operating system, the list is scanned to find a suitable region. The rectangle with the smallest area greater than or equal to the required number of user blocks to be mapped is selected and this rectangular area is allocated for the mapping process. If there is no such rectangles with enough size, than we select the largest rectangle (the first rectangle in the list) and perform the above search procedure for the remaining number of unallocated user blocks. This process continues till the number of unallocated user blocks area is zero. The pseudo code for the region allocation process is given in Figure 6.7. 


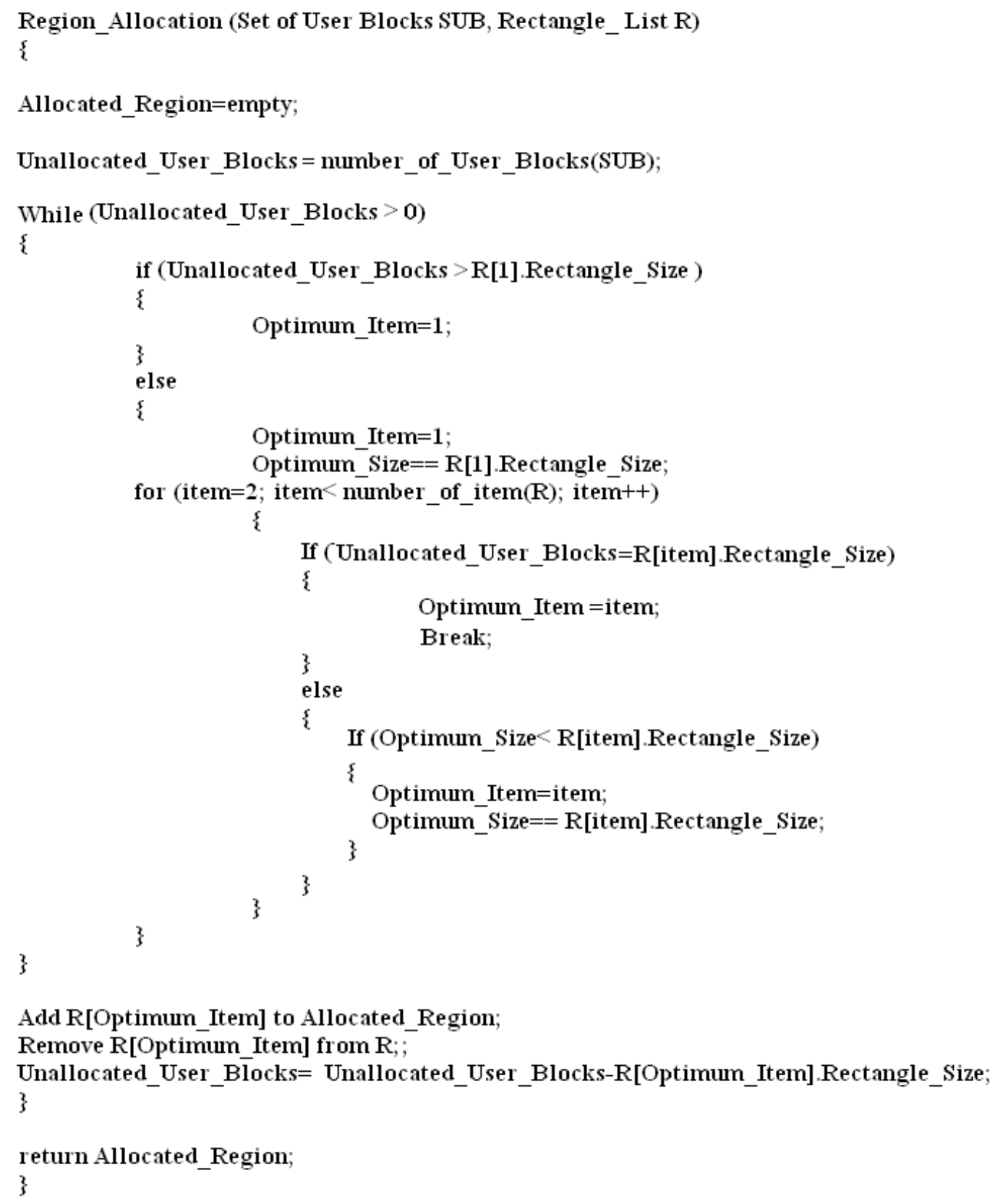

Figure 6.7: Pseudo code for region allocation phase 


\subsubsection{Phase-2: User Block Placement}

In this phase the user blocks are physically mapped onto the allocated region. This mapping is done to minimize both the Manhattan distance and the path blocking rate. Since the access point load balancing is taken into account in Phase-1, it is not considered in this phase.

As was mentioned above, the problem of minimizing the Manhattan distance is NP-complete. Because mapping decision has to be given in run time in a very short duration, the following ad-hoc mapping technique is presented. The mapping is done in a best fit manner by taking both the Manhattan distance and path blocking into account.

The following procedure is applied when the allocated region is not a single but a multiple of rectangles formed by free user blocks;

- Sort user blocks with respect to descending traffic demand. This traffic demand is the sum of all $S_{D O U T}$ traffic, i.e. all user block to user block traffic involving the current user block.

- Place the first user block in the list onto the middle free user block of the largest rectangle on the allocated area.

- Starting from second user block in the list, place it onto the free user block such that;

- If there is a free user block on the current rectangle;

* The Manhattan distance from this user block to the middle of the current rectangle area is minimized.

* If there is more than one free location, select the one minimizing the path blocking rate.

- Otherwise place the user block to the middle of the next largest rectangle and proceed with this new rectangle for the next user blocks.

If the allocated region is a set of free user blocks forming a rectangle, then the following two step mapping is performed. In the first step, the following procedure is used to form a virtual rectangular area. 
- Sort user blocks with respect to descending traffic demand. This traffic demand is the sum of all $S_{\text {DOUT }}$ traffic, i.e. all user block to user block traffic involving the current user block.

- Form a virtual rectangle composed of a single user block by placing the first item in the list.

- Starting from the second user block in the list, expand the virtual rectangle by adding this candidate user block for placement. The candidate user block location for this expansion must be chosen so that the virtual rectangle can fit into the allocated rectangle. Among these expansion alternatives select the one such that;

- The Manhattan distance from this candidate user block to the user block in the virtual rectangle area having the largest traffic demand is minimized.

- If there are more than one expansion alternatives, select the one minimizing the path blocking rate.

Following the above virtual area forming process, the virtual rectangle is placed onto the allocated rectangle as the second step. Note that, the placement may require $90^{\circ}$ rotation of the virtual rectangle. In Figure 6.8, the virtual rectangle forming steps and its physical mapping are shown assuming that 6 user blocks to be mapped onto a region with $2 \times 4$ user blocks.

The list of free rectangular areas used in phase- 1 needs to be updated after this placement phase. When the execution of an already mapped user block is completed, this list also needs to be updated. Partitioning a free area into a set of rectangles is a well studied field [12][13] [14]. The rectangle list generation process proposed by $\mathrm{Gu}$ et al. [14] is suitable for hardware implementation and this technique is chosen as the free rectangle list generation method in our mapping technique.

\subsection{Quality Analysis for The Mapping}

Due to NP-completeness of the mapping problem $P_{m}$ an ad-hoc mapping technique is developed in the previous section. In order to show how well our ad-hoc mapping technique is, some comparisons are made. Since it is not know yet whether it is possible to solve $P_{m}$ 


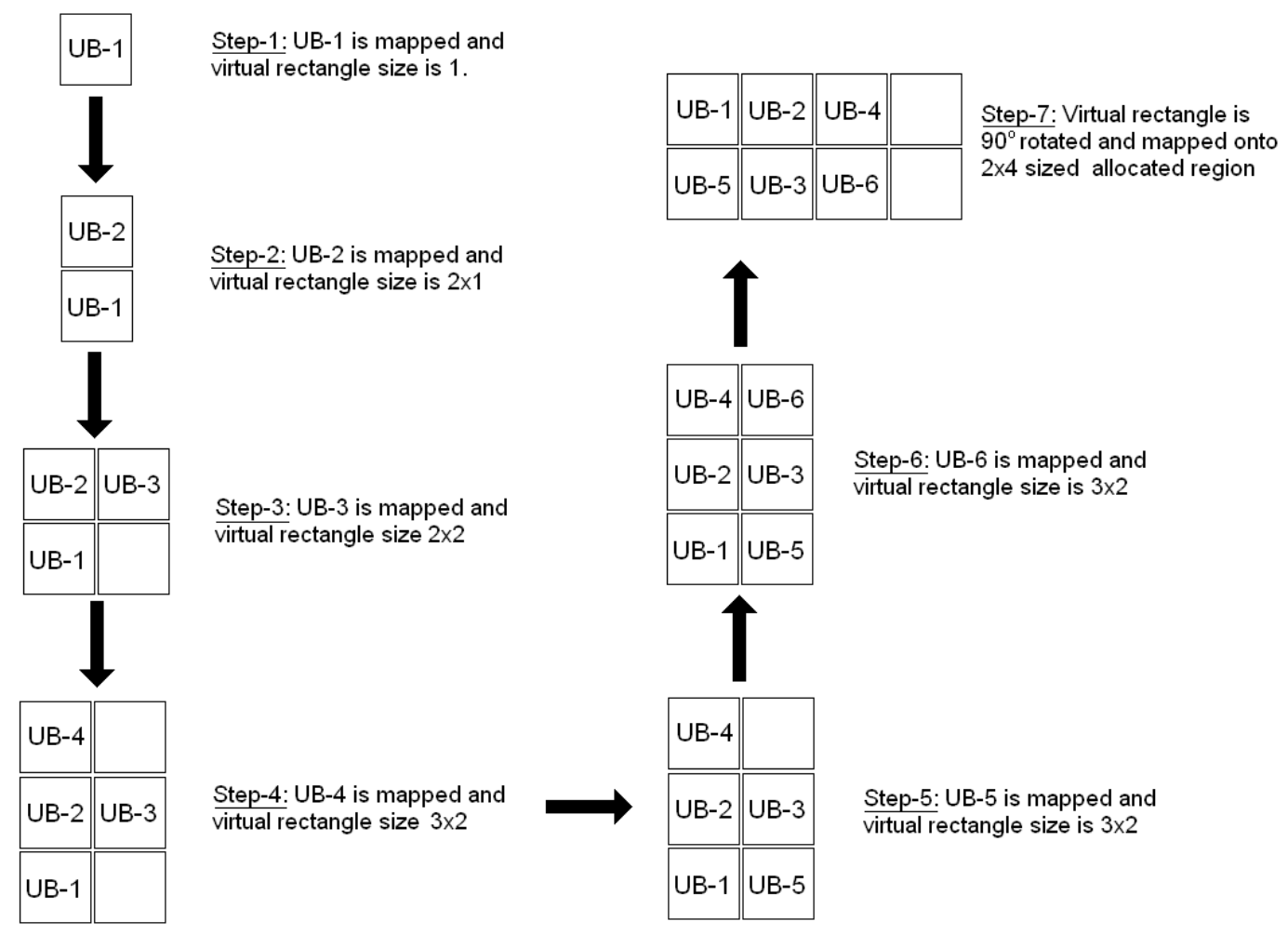

Figure 6.8: A virtual rectangle based mapping example

optimally is impossible in polynomial time or not, we performed comparisons using solutions taking just one objective into consideration at a time.

In order to find the average gap between solutions provided by our mapping technique and single objective solutions, the following comparative study has been carried out. In each of the comparative studies, 1000 test cases are created by randomly generating 100 set of user blocks having data dependency for each case. The number of user blocks is selected uniformly between 1 and 9 randomly. For each user block, random traffic is generated to at most half of the user block with a rate randomly selected within the range of 1MByte/s to 20MByte/s. In a similar manner, memory read and write demands are randomly generated between 10MByte/s to $50 \mathrm{MByte} / \mathrm{s}$. The reconfigurable hardware surface is assumed to be partitioned into $10 \times 10$ user blocks. If there is not enough free space when a set of user blocks with data dependency is scheduled, the execution of the earliest scheduled set is assumed to be completed and these user blocks are removed from the reconfigurable hardware.

The single objective solution just minimizing the Manhattan distance is found in a brute force 
manner by mapping the set of user blocks onto completely empty reconfigurable hardware surface and calculating the sum of Manhattan distance between communicating pairs. The single objective solution for minimum total path blocking rate is found in a brute force manner, too. When the reconfigurable device usage is kept at $50 \%$, our ad-hoc mapping performs pretty well and the sum of total Manhattan distance is just $22 \%$ larger than that of the single objective function case. Similarly, the total path blocking rate is kept 33\% larger than that of single objective function case. When the device usage is increased to $70 \%$ for moderate device usage, the Manhattan distance become $46 \%$ larger than the single objective function case and the total path blocking rate is $58 \%$ larger than that of the single objective function case. This is due to fact that, as the device usage increases, the probability of fragmentation and hence the probability of allocating non-contiguous area increases. If the device usage is increased to $90 \%$, our ad-hoc mapping achieves $102 \%$ and $128 \%$ larger sum of total Manhattan distance and total path blocking rate when compared to single objective function case, respectively. For a fully loaded device usage, the sum total Manhattan distance increases further and become $157 \%$ larger than that of single objective function case. Similarly, the total path blocking rate is around 3 times that of the single objective function case for a fully loaded device usage condition.

The single objective function to minimize the standard deviation of the access point loads maps the set of user blocks having data dependency onto non-contiguous region and find the solution in a brute force manner. For a fully loaded device usage condition, the standard deviation of the access point traffic load is 3.2 times that of single object function case that considers access point load balancing only.

Another comparative study with this data set and execution scenario is performed with a random mapping. Since the mapping itself takes some time and requires logic blocks for its implementation, this comparative study is made to show the improvement as compared to random mapping, which has no latency and resource requirement. When the device is fully loaded, the sum of Manhattan distance achieved with the random mapping is 4.8 times that of our ad-hoc mapping. The random mapping results in a 12.7 times sum of total Manhattan distance and 2.7 times the standard deviation of the access point traffic load when compared to our ad-hoc mapping proposal. From this results, it is obvious that we need the propose ad-hoc mapping circuitry in our hardware operating system. 


\section{CHAPTER 7}

\section{HARDWARE OPERATING SYSTEM}

In a conventional computing platform built with a GPP, the details of the underlying hardware are hidden from the application programmer by the operating system. In addition to task scheduling, the operating system manages the computing platform resources such as memory, hard drives, I/O interfaces and provides high level services such as file system, networking and inter-task communication. The operating system in our reconfigurable computing platform has similar objectives, too.

As was mentioned in Chapter 3, our reconfigurable computing platform is based on a reconfigurable hardware and a GPP and hence supports both hardware and software tasks. The proposed operating system is composed of three parts. The first part is a commercially available real time operating system that can give all the necessary support for software tasks. The second part is the hardware operating system software running on GPP. This part makes the necessary connection between the reconfigurable hardware and the commercial operating system. Finally, the hardware operating system circuitry is responsible for the management of the reconfigurable hardware resources for scheduling of hardware tasks and for providing high level services to hardware tasks.

The high level operating system service request of hardware tasks are simply forwarded to the commercial operating system using both the hardware operating system circuitry and the software. This forwarding is done whenever a SYS_CALL message is sent by the hardware task over the IBCN. In a similar way, the service reply is sent by a TASK_CALL message over the IBCN. Therefore, the two main duties of the hardware operating system can be listed as the management of the reconfigurable hardware and the scheduling of the hardware tasks. Since the application programmer should no longer worry about the underlying hardware, 
the only requirement is to provide the hardware tasks in the form of a set of well partitioned execution blocks and the accompanying task dependency graph.

In this chapter, our novel offline bitstream generation process is discussed first.In this process,the set of hardware tasks in the form of execution blocks is the input and the associated bitstream for the reconfigurable hardware task is the output. The details of our hardware operating system are presented afterwards.

\subsection{Offline Bitstream Generation}

In conventional computing systems, the application programs are converted to binary files and these files are created by compilers depending on the operating system and GPP instruction set. A similar analogy exists in our reconfigurable computing platform and the user applications that will execute on the reconfigurable hardware have to be converted into bitstream files based on the reconfigurable device logic block architecture.

The reconfigurable computing platform developed in this thesis work targets embedded applications. In embedded applications, system requirements are known for the whole system prior to field operation. Therefore, in this subsection, we propose an offline design flow to generate the bitstream for the given hardware tasks with the aim of maximizing the overall system performance. In addition, the bitstream for static circuits (IBCN, Host\&Memory Interface and Hardware Operating System) are also generated during this process.

The bitstream generation process starts with the given set of hardware tasks, which are defined in terms of dependency graphs and execution blocks assuming that execution blocks are modeled in a hardware description language (HDL) such as VHLD or Verilog. In the first stage, a commercial HDL synthesis tool is used to find the resource requirements and execution time of each execution block in the system.

Then, by using the user-defined device utilization factor $\beta_{j}$ parameter for each hardware task $j$ and IBCN payload size, we run our greedy method to find the optimal block size and partitioning parameters.With these partitioning parameters the bitstream for the static circuits can now be generated using a commercial synthesis, placement and routing (PAR) tool.

In the next stage, the execution blocks are packet into a number of user blocks for each hard- 
ware task. The output of the packing process is the reduced hardware task $H T_{j}^{\prime}\left(S_{U B}, G^{\prime}, D\right)$ for each hardware task j. Finally, the commercial synthesis and PAR tool can again be used to generate the position independent dynamic bitstream for the user blocks of each hardware task.

A summary of the above process is depicted in Figure 7.1.

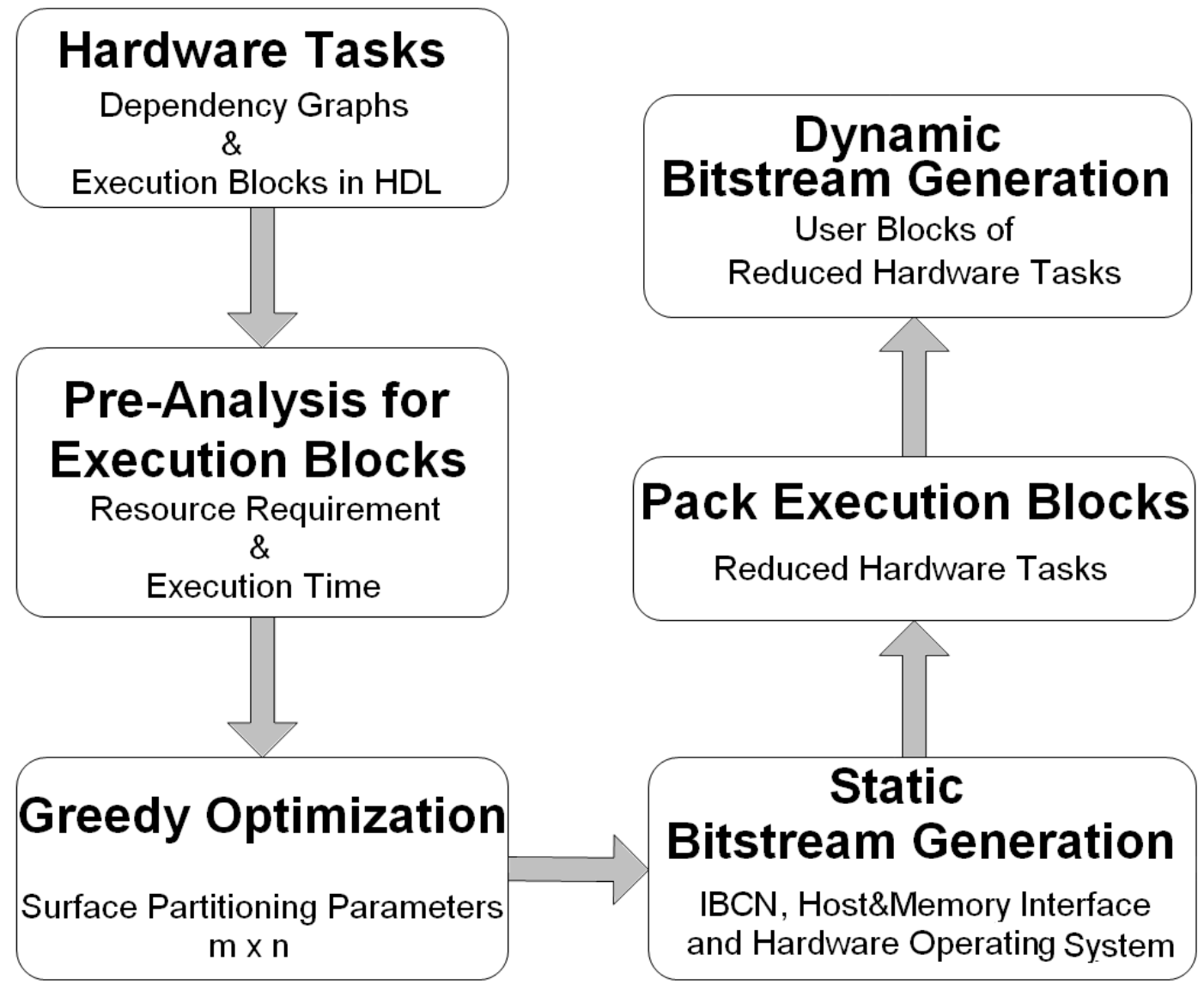

Figure 7.1: Offline bitstream generation process

\subsection{Hardware Operating System Components}

The hardware operating system in our proposed reconfigurable computing platform is a collection of functional building blocks. Some of them are a pieces of software running on the GPP and the other ones are hardware circuitry operating on the reconfigurable device. 
In Figure 7.2, the organization of these functional building blocks with their connections is presented. Although not shown in the figure, there is a communication path to external memory from all hardware blocks except host communication controller. Below, each functional building block is discussed in detail.

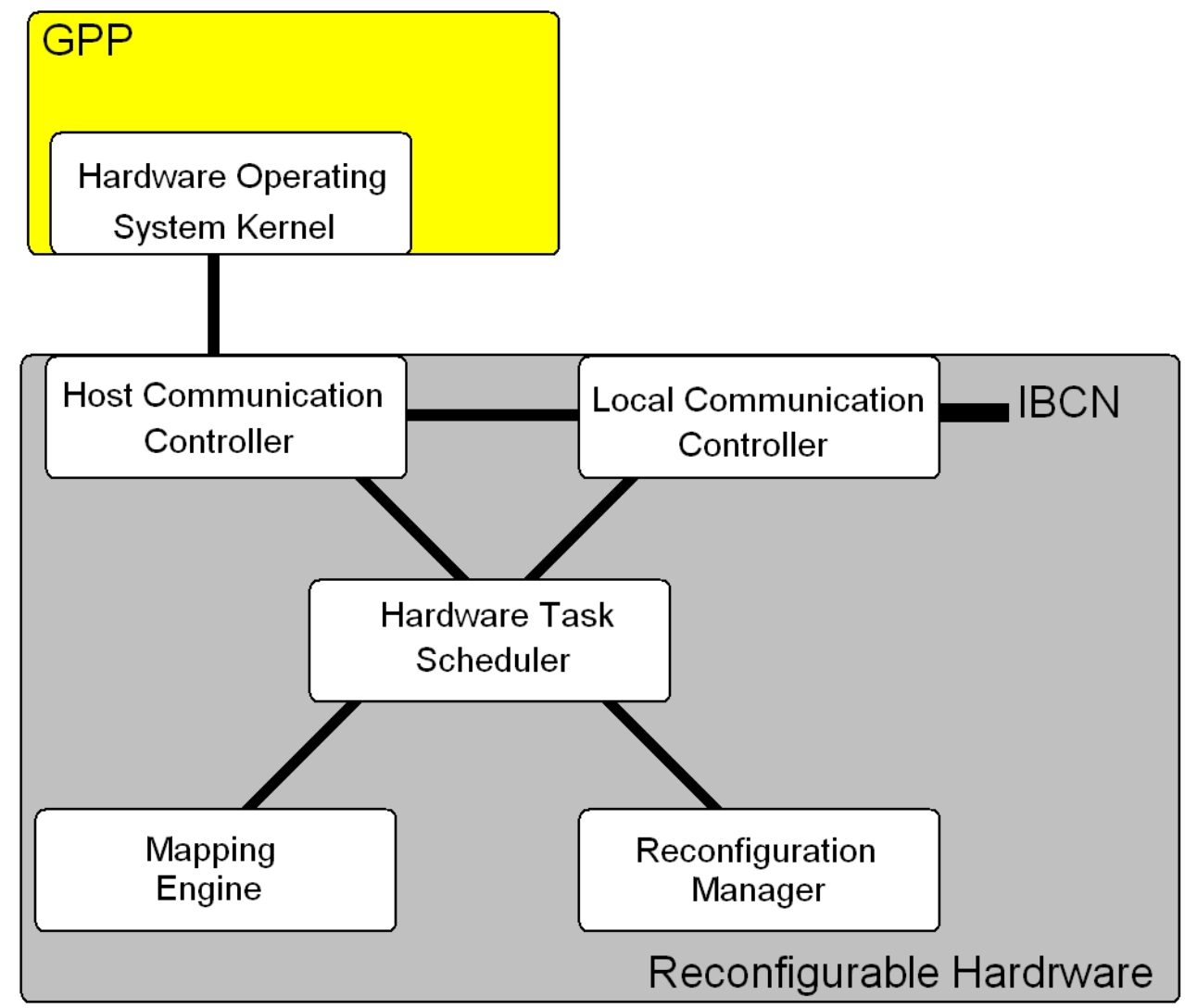

Figure 7.2: Hardware operating system components

\subsubsection{Hardware Operating System Kernel}

This part of the hardware operating system is a piece of software task running on the GPP. Operating in the kernel mode of the commercial operating system, this task has high priority as compared to other software tasks in order to give a faster response. Whenever a hardware task is spawned by the commercial operating system, this software creates a ghost task in the commercial operating system task list. In this way, the spawned hardware task is seen as an ordinary software task to the rest of the system. Then this spawn request is forwarded to the 
hardware task scheduler. Whenever a hardware task completes its execution, the hardware operating system kernel is informed and it simply kills the ghost task.

In addition to handling the hardware task spawning issue, the hardware operating system kernel is responsible to give high level services to the hardware tasks. If a service request is received from the hardware task, the ghost task is simply called to get the required service. The ghost task sends the result of this service request to the hardware operating system kernel which is then forwarded to the hardware task. A similar mechanism is used for inter-task communication also. Since the inter-task communication requires operating system services, the communication between two hardware tasks is handled by the communication between the associated ghost tasks.

\subsubsection{Host Communication Controller}

This circuitry is responsible for providing the communication path between the GPP and the reconfigurable hardware. Its architecture strongly depends on the underlying hardware and the coupling between the GPP and the reconfigurable hardware. The message received from the hardware task controller and local communication controller is simply forwarded to the hardware operating system kernel over the host interface. Whenever a message is received from the hardware operating system kernel over the host interface, it is forwarded to;

- Hardware task scheduler if it is a task spawn message

- Local communication controller if it is an operating system service request/response to a hardware task

\subsubsection{Local Communication Controller}

In order to connect the IBCN and hence the hardware tasks to the rest of the system, the local communication controller circuitry is used. The messages received from the IBCN are forwarded to;

- Hardware task scheduler if it is a FINISH SIGNAL message,

- Host communication controller if it is SYS_CALL message, 
- Memory Controller if it is a REQUEST or M_WRITE message.

In a similar fashion the messages are forwarded to IBCN if it is a;

- START SIGNAL message from hardware task scheduler,

- TASK_CALL message from the host communication controller,

- $M_{-} R E A D$ message from the memory controller.

\subsubsection{Hardware Task Scheduler}

After the bitsteams are generated using the proposed offline bitstream generation process, the embedded system becomes ready for its field operation. The field operation starts by configuring the bitstream associated with the static circuits first. Then the reduced hardware task graph and the bitstream of user blocks of all hardware tasks are loaded into memory. From this point onwards the hardware task schedule is responsible for managing the reconfigurable hardware surface. Whenever the user schedules a hardware task using the commercial operating system, the hardware task scheduler loads the necessary user block bitstreams to perform the computation.

A number of hardware task schedulers were already proposed in the literature $[14,29,31,33,34]$ which were reviewed in Chapter 2. All of these schedulers were custom designed and had specific characteristics related to their target computing platforms including their hardware task models and surface partitioning techniques. Therefore a hardware task scheduler specific to our computing platform is also presented in this thesis work. This scheduler is designed to support true multitasking with priority based scheduling for both soft and hard real time embedded systems.

In the proposed reconfigurable computing platform, thanks to our novel hardware task model, whenever a higher priority hardware task arrives, there is no need to save the hardware state of a lower priority task for preemption, as compared to conventional computing. If there is not enough free space on the reconfigurable hardware surface, this high priority task waits in the priority queue for enough number of user blocks to complete their execution but not for the completion of a whole hardware task because a hardware task is assumed to be composed 
of a number of user blocks. The user block execution time is much smaller than the total execution time of a hardware task and hence it is highly probable that enough free area becomes available in a very short time. In this way, the hardware task scheduler supports soft priority.

In addition to soft priority based scheduling, the hardware task scheduler is designed to support hard real time scheduling also. A hard real time hardware task is characterized by its deadline $D$, which defines the maximum time interval between its scheduling and its completion. In the literature, whenever a hard real time hardware task with a specific deadline $D$ is scheduled, the scheduler either accepts or rejects the scheduled hardware task depending on the status of the system $[14,70,71,72]$. A similar acceptance test is performed by our hardware task scheduler. The proposed hardware task scheduler checks the current state of the reconfigurable hardware and the priority queue to determine whether the deadline $D$ can be met or not. The hardware task is then put into the priority queue and acceptance information is returned to the commercial operating system if the deadline can be met, otherwise the rejection information is returned. In the proposed reconfigurable computing platform it is the user's responsibility to take care of this rejection if hard real time support is needed.

In order to perform scheduling, the hardware task scheduler works in conjunction with the mapping engine and the reconfiguration manager. The state of a hardware task spawned by the commercial operating system is kept in the unified memory with a special data structure. A hardware task is represented using a linked list of user block node data structure as shown in Figure 7.3.

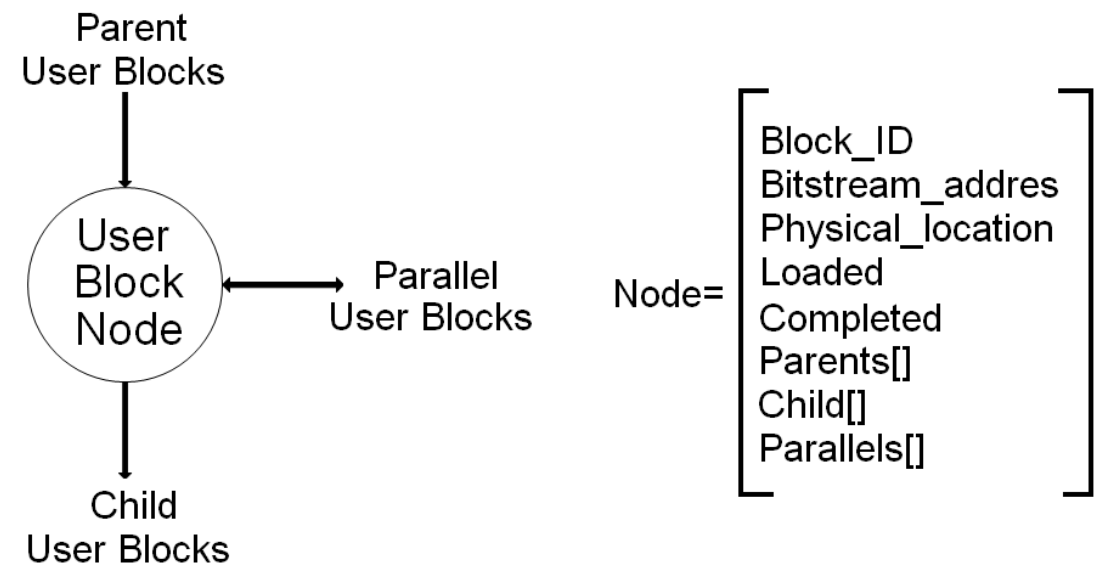

Figure 7.3: Node structure in the hardware task representation 
In the user block node structure, the field

- Block_ID identifies the user block,

- Bitstream_address represents the location of the associated bitstream in the memory,

- Physical_location represents the user block where the associated bitstream is loaded on the reconfigurable hardware surface,

- Loaded represents the information whether the user block bitstream is loaded onto reconfigurable hardware or not

- Completed represents the information whether the user block execution is completed or not,

- Parents[] is a pointer array holding pointers to user blocks after which current user block will start execution ,

- Childs[] is a pointer array holding pointers to user blocks that will start execution after the completion of the current user block,

- Parallels[] is a pointer array holding pointers to user blocks that executes in parallel with the current user block.

At power up, a linked list is created in the memory for each hardware task based on its reduced task graph representation. Whenever a hardware task is spawned and it is accepted by the hardware task scheduler, the Loaded and Completed fields of all user blocks are set to FALSE and the Physical_location fields are set to NULL position. Then the first user block is put into the priority queue, which handles the scheduling in a first-in-first out scheme.

The hardware task scheduler continuously checks this priority queue and the free number of user blocks on the reconfigurable hardware surface. If there is enough room, the hardware task scheduler issues a mapping request to mapping engine, which fills the Physical_location fields of the user blocks in the Parallels[] array of the current user block. After this mapping completed, the hardware task scheduler triggers the reconfiguration manager to load the bitstream in the memory location of Physical_location field to the free user block addressed by Physical_location field. After this context loading process is completed, the hardware task scheduler sends START SIGNAL to loaded user blocks. Then this user block is removed from 
the priority queue and the first user block in the Childs[] array, which represents next level of the reduced hardware task, is placed onto the priority queue. For the second level user block the mapping and context loading procedures are applied similar to the first level user blocks. In this way the pre-fetching of the next stage takes place minimizing the reconfiguration time overhead. However, no START SIGNAL is sent to this second level user block.

Whenever a FINISH SIGNAL is received from a user block, the hardware task scheduler first informs the mapping engine. Then it sets the Completed field to TRUE and checks the Completed field of the user blocks in the Parallels[] array of the current user block. If all user blocks have completed their operations, the hardware task scheduler checks whether the pre-fetching is completed by using the Loaded field of the user blocks pointed by the Childs[] array. If all user blocks of the next level is loaded, the hardware task scheduler then sends the START SIGNAL to loaded user blocks in the Childs[] array, adds the first user block of the next level Childs[] array into the priority queue for pre-fetching again. If all user blocks are not loaded, this execution start and pre-fetching process waits until all loading is completed.

\subsubsection{Mapping Engine}

This circuit is the hardware implementation of the mapping technique presented in Chapter 6. The mapping engine is responsible for keeping the usage information of the reconfigurable hardware surface. Hence, an $n \times m$ array is kept in the memory to indicate whether a user block is free or not. In addition, a list of free rectangles is kept in memory for phase- 1 of our mapping technique.

The mapping engine starts its operation with a trigger from the hardware task scheduler. The user blocks to be mapped are known using the Parallels[] array of the user block given by the hardware task scheduler. If there is enough free space, mapping engine maps the user blocks by filling the Physical_location fields of the user blocks. If there is not enough free user blocks, the mapping is pended until enough free space is available.

When mapping is completed, the mapping engine updates the $n \times m$ array and the list of free rectangles in the memory. This update is also performed whenever a trigger from the hardware scheduler due to the completion of a user block is received. 


\subsubsection{Reconfiguration Manager}

The partial reconfiguration interface of the underlying reconfigurable device is used by this circuitry. The reconfiguration manager starts partial reconfiguration of the reconfigurable device with a trigger from the hardware task scheduler. The user block provided by the hardware task scheduler is loaded first. Just after partial reconfiguration of the user block is completed, after fetching the bitstream from the Bitstream_address field and loading it to the physical location addressed by Physical_location field, the Loaded field is set TRUE and the hardware task scheduler is informed. In this way the execution of this user block can start without waiting for other user blocks and hence an improvement in the reconfiguration time overhead is achieved. The partial reconfiguration of the user blocks in the Parallels[] array is done in the same manner. 


\section{CHAPTER 8}

\section{IMPLEMENTATION OF THE RECONFIGURABLE COMPUTING PLATFORM}

In order to evaluate the performance of our reconfigurable computing platform proposal, all building blocks are implemented. Since part of the reconfigurable hardware is used for these building blocks, the first performance criterion is the number of logic blocks needed. The second one is the operating frequency or the execution time of these building blocks.

For the implementation, Xilinx Virtex6 LX760 series FPGA is used and it is assumed that it is coupled with an external GPP. This FPGA is a run time partially reconfigurable device with the constraint of reconfiguring a minimum 40 CLBs in a column. In this chapter, the implementation of the proposed IBCN architecture is given first. Then, the mapping technique is implemented and its performance is reported. Finally, the implementation results of our complete reconfigurable computing platform is given using this FPGA. Note that logic block requirements and operating frequency for a given circuit is obtained using the Xilinx ISE 12.4 [73] tool. Similarly, the execution times are obtained with the embedded simulator in the ISE tool.

\subsection{IBCN Implementation Results}

In the proposed IBCN architecture, two parameters are user defined. These are the payload size and the depth of the FIFO and these parameters are in powers of two in general. As was mentioned earlier, the payload size must be chosen such that it can address any user block on the reconfigurable device surface, i.e. $2^{\text {payload_size }} \geq(m \times n)$. Since IBCN requires extra two bits to identify flit type, the word length and hence the flit size becomes $(2+$ payloadsize $)$. 
As was mentioned earlier, the FIFO depth is a critical factor affecting path blocking probability due to wormhole switching and has an impact on overall system performance. Therefore, the deeper the FIFO, the smaller the path blocking probability is. However as FIFO becomes deeper, the operating frequency decreases and the logic block demand increases. In order to show the effect of these user selected parameters, the building blocks and the whole IBCN switch has been implemented using Xilinx Virtex6 LX760 series FPGA for payload sizes of 8 and 16 and FIFO depths of 8 and 16.

The implementation results for just a single FIFO is given in Table 8.1 for the combinations of selected payload and depth values. The logic requirements are given in terms of available reconfigurable logic slice flip flops (FF) and look up tables (LUTs). Note that in this FPGA architecture, flip flops are used for local storage and look up tables are used for gate level implementations. As expected, logic requirements are directly related to word width and FIFO depth, whereas the operating frequency is just related to FIFO depth.

Table 8.1: IBCN FIFO implementation

\begin{tabular}{|c|c|c|}
\hline Width, Depth & Logic Requirements & Operating Frequency(max) \\
\hline 10bit, 8 word & Slice FF pairs : 99 out of 948480 & $867 \mathrm{MHz}$ \\
\hline & Slice LUTs : 30 out of 948480 & \\
\hline 10bit , 16 word & $\begin{array}{l}\text { Slice FF pairs : } 197 \text { out of } 948480 \\
\text { Slice LUTs : } 63 \text { out of } 948480\end{array}$ & $671 \mathrm{MHz}$ \\
\hline 18bit, 8 word & $\begin{array}{l}\text { Slice FF pairs : } 171 \text { out of } 948480 \\
\text { Slice LUTs : } 45 \text { out of } 948480\end{array}$ & $867 \mathrm{MHz}$ \\
\hline 18bit , 16 word & $\begin{array}{l}\text { Slice FF pairs : } 341 \text { out of } 948480 \\
\text { Slice LUTs : } 103 \text { out of } 948480\end{array}$ & $671 \mathrm{MHz}$ \\
\hline
\end{tabular}

In the next step, virtual channel are implemented using two FIFOs, multiplexer and the necessary control logic. The implementation results of virtual channels with the above user provided parameters given in Table 8.2 below.

The next implementation is done for the control logic of the IBCN, which keeps the wormhole routing tables and makes necessary connections and updates according to received flit's content. As reported in Table 8.3, the operating frequency is independent of the word width and the control logic has slower operating frequency compared to FIFOs due to large propagation delay. 
Table 8.2: IBCN virtual channel FIFO implementation

\begin{tabular}{|c|c|c|}
\hline Width, Depth & Logic Requirements & Operating Frequency(max) \\
\hline 10bit , 8 word & $\begin{array}{l}\text { Slice FF pairs : } 203 \text { out of } 948480 \\
\text { Slice LUTs : } 75 \text { out of } 948480\end{array}$ & $857 \mathrm{MHz}$ \\
\hline 10bit , 16 word & $\begin{array}{l}\text { Slice FF pairs : } 392 \text { out of } 948480 \\
\text { Slice LUTs : } 131 \text { out of } 948480\end{array}$ & $625 \mathrm{MHz}$ \\
\hline 18 bit , 8 word & $\begin{array}{l}\text { Slice FF pairs : } 355 \text { out of } 948480 \\
\text { Slice LUTs : } 115 \text { out of } 948480\end{array}$ & $857 \mathrm{MHz}$ \\
\hline 18bit , 16 word & $\begin{array}{l}\text { Slice FF pairs : } 691 \text { out of } 948480 \\
\text { Slice LUTs : } 211 \text { out of } 948480\end{array}$ & $625 \mathrm{MHz}$ \\
\hline
\end{tabular}

Table 8.3: IBCN switch control logic implementations

\begin{tabular}{lll}
\hline Width & Logic Requirements & Operating Frequency(max) \\
\hline 10bit & Slice FF pairs : 661 out of 948480 & $515 \mathrm{MHz}$ \\
& Slice LUTs : 661 out of 948480 & \\
18bit & $\begin{array}{l}\text { Slice FF pairs : 879 out of } 948480 \\
\text { Slice LUTs :879 out of } 948480\end{array}$ & $515 \mathrm{MHz}$ \\
\hline
\end{tabular}

Finally, the above building blocks are used to implement the complete IBCN switch. As shown in Table 8.4, the operating frequency of the whole switch itself is further slowed down due to larger wire lengths and propagation delays. These logic requirement and the operating frequency figures can be used to evaluate the performance of our IBCN proposal. For example, the area overhead for IBCN turns out to be 13\% for 10x10 surface partitioning and 10bit/8word IBCN architecture with an aggregate bandwidth of 62.4 GByte/s.

Table 8.4: IBCN switch implementations

\begin{tabular}{lll}
\hline Width , Depth & Logic Requirements & Operating Frequency(max) \\
\hline 10bit , 8 word & $\begin{array}{l}\text { Slice FF pairs : 1239 out of } 948480 \\
\text { Slice LUTs : 818 out of } 948480\end{array}$ & $390 \mathrm{MHz}$ \\
10bit , 16 word & $\begin{array}{l}\text { Slice FF pairs : 1739 out of } 948480 \\
\text { Slice LUTs :1029 out of } 948480\end{array}$ & $354 \mathrm{MHz}$ \\
18bit , 8 word & $\begin{array}{l}\text { Slice FF pairs : 1827 out of } 948480 \\
\text { Slice LUTs :1111 out of } 948480\end{array}$ & $390 \mathrm{MHz}$ \\
18bit , 16 word & $\begin{array}{l}\text { Slice FF pairs : 2751 out of } 948480 \\
\text { Slice LUTs :1488 out of } 948480\end{array}$ & $354 \mathrm{MHz}$ \\
&
\end{tabular}




\subsection{Mapping Engine Implementation Results}

In this reconfigurable computing platform, as was discussed in Chapter 6, a two phase mapping technique is used to decide the physical locations of a given set of user blocks. The duty of second phase is to place the user blocks onto the allocated region, which is the output of the first phase. Therefore, there is not a tight coupling between these two stages. In a similar way, the first phase works on a free rectangle list generator circuitry, which can be implemented separately too. In this subsection, the implementation details for these three circuitry are discussed in details.

\subsubsection{Free Rectangle List Generator Circuitry}

The list of free rectangles can be generated in two ways. In the first method; starting from a single rectangle, which represents the whole reconfigurable hardware surface, smaller rectangles are created upon allocating some parts to hardware tasks $[12,13]$. This rectangle list is updated either by merging or creating new rectangles whenever an already allocated region completes its execution. The second alternative [14] is to generate the list every time requested by phase- 1 of the mapping engine. Since the list is already available in the first case, phase-1 can start its operation immediately. However the update requires time and if an update is in progress, phase-1 should wait until it is completed. Indeed, the update operation may result in a few small sized rectangles although it was possible to merge them into a bigger single rectangle. In our computing platform, it is expected to have very frequent changes on the reconfigurable hardware surface because the small sized execution blocks are placed in and out instead of the whole hardware task circuitry. Therefore, it will be better to generate the list each time it is needed.

In [14] a very efficient technique was proposed and it was reported that on average, the list generation takes about 70 nanoseconds on a $20 \times 36$ grid surface. Thanks to our packing process, the reconfigurable hardware surface is partitioned into coarse grained regions. In addition, the IBCN logic requirement is a limiting factor and it is not feasible to partition the device into too small sized user blocks. For example, when Xilinx Virtex6 LX760 FPGA is partitioned into $20 \times 20$ user block area, the IBCN requires around half of the FPGA logic resources. Since the proposed heuristic to find partitioning parameters takes also IBCN logic 
requirements into account, the small sized user blocks and hence large number of $m$ and $n$ values will not be observed. Therefore, generating the free rectangle list online instead of keeping and updating is a much more feasible approach.

As depicted in Figure 8.1, the free rectangle list generator is composed of an $m \times n$ array to keep the information of whether the associated user block is free or not. Exactly the same logic implementation in [14] is used to search the free rectangles by reading the device usage information over this array. This array is updated whenever there is a change on the device usage. If an already filled user block completes its execution it sends the FINISH SIGNAL and the hardware task scheduler informs the user block usage updater logic. In a similar fashion, whenever the phase- 2 of the mapping engine decides to use a user block, the array is updated. Note that no update is performed if a list generation is in progress. Similarly, the search engine must wait till the updates are completed. The rectangle search engine writes the rectangle information in a pipelined manner while the search is in progress.

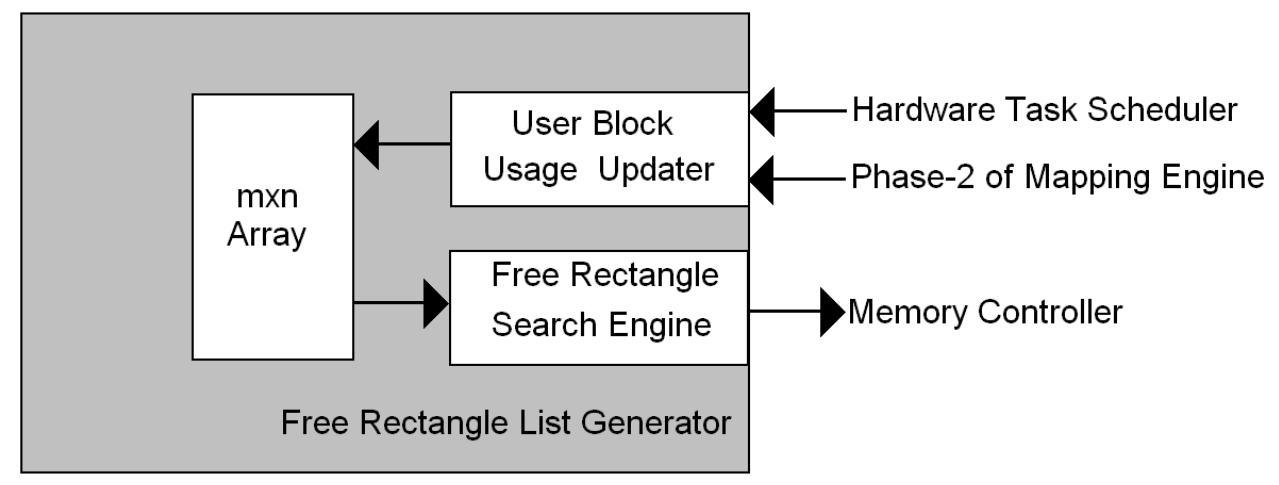

Figure 8.1: Free rectangle list generator block diagram

This circuitry is implemented for the Xilinx Virtex6 LX760 FPGA partitioned into $10 \times 10$ user blocks. With an operating frequency of $308 \mathrm{MHz}$, the logic requirements are just 1856 flip flop out of 948480 and 2244 look up tables out of 948480 , which is less than $0.25 \%$ of the available logic resources. The rectangle search time strongly depends on the current device usage information. In the best case, when the whole surface is empty the search takes just 63 nanoseconds. The worst case search time is 325 nanoseconds if the free rectangles are all single user blocks as shown in Figure 8.2. Note that in these timing results, the posted memory write access are not included. 


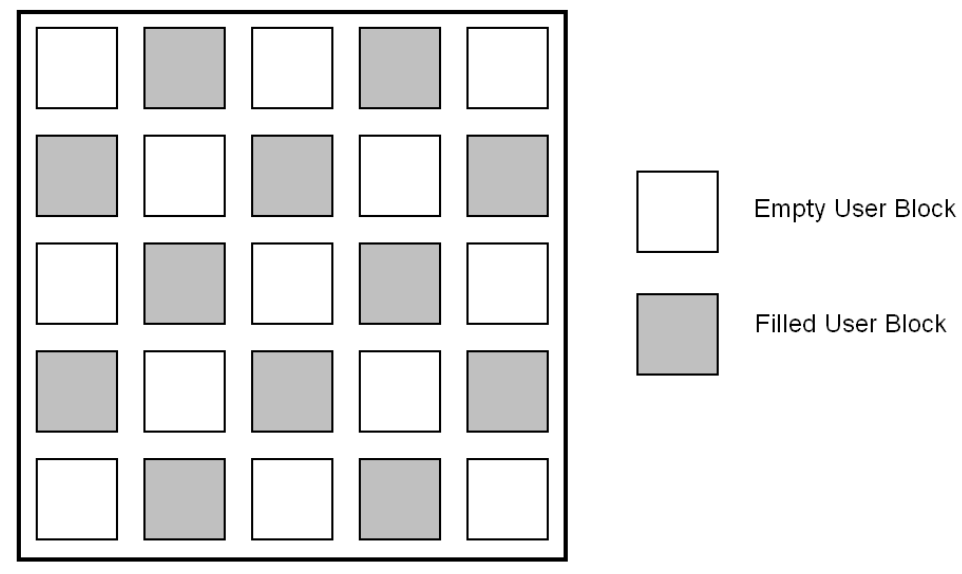

Figure 8.2: Worst case condition for free list generation circuitry

\subsubsection{Phase-1 Circuitry}

The functionality of this circuitry is to allocate the most suitable rectangle(s) to a given number of user blocks. Since the list of free rectangles is kept in memory, phase-1 circuitry just inputs the number of user blocks needed from the hardware task scheduler. Whenever a region allocation request is made, this circuitry starts to allocate a region from the sorted rectangle list and selects the rectangle(s) in a best fit manner. The rectangle select operation just requires scanning of this sorted list and, depending on the required number of user blocks, it can be finished in a few scan operations.

However, having a sorted rectangle list, according to the sorting rules given in Chapter 6, requires a much more complex circuitry. Although it is possible to start from a sorted list and update it with the changes on the reconfigurable device surface, such an implementation will be more complex. Since the number of user blocks and hence the number of free rectangles are limited, the sorting process can be repeated every time it is needed with a simpler circuitry.

On average the well known sorting techniques, like merge sort and insertion sort, have $O(n \log n)$ complexity. During the sorting process, there is no need to insert a free rectangle into the list if there are better sized rectangles. For example, if the required area size is 8 user blocks and the list already contains rectangles that are greater or equal to 8 then there is no need to put a rectangle with size less than 8 into the list and seek for the suitable position for it. In this way, the sort complexity will become $O(n)$ and just a single flag is sufficient to indicate whether 
a rectangle with size less than or equal to the requested user block number is already in the list or not. As sorting requires $\mid$ width - length $\mid$ and access point load comparisons, adder and subtraction blocks are needed in this circuitry.

Although the achieved operating frequency of this circuit is $433 \mathrm{MHz}$, due to memory read request, the operating frequency is limited by the memory controller used for the storage of the free rectangle list and sorted list. With today's dynamic memory architectures it is possible to provide high bandwidth if the memory access occurs at consecutive addresses. But for random and single word read/write access too much time is lost due to initial latency. However, our sorting process requires read and writes to random locations. Therefore the operating frequency of our circuitry will be very limited if such a dynamic memory is used. Thanks to FPGAs embedded static RAM based dual port block RAM implementations operating at $400 \mathrm{MHz}$, it is possible to operate the circuit at 400MHz. For this specific FPGA, the logic requirement of the region allocation process is 1648 flip flops out of 948480, 4456 look up tables out of 948480 and 1 block RAM out of 1,440. As shown in Figure 8.3, the other port of the block RAM is used as the memory interface for free rectangle generator circuitry. As the allocated region is written to a memory for phase- 2 access, a separate memory interface is needed.

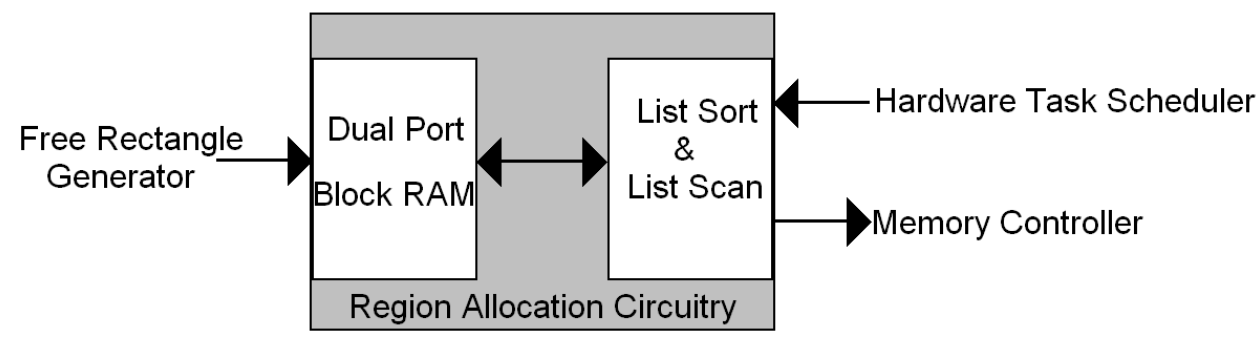

Figure 8.3: Region allocation circuitry block diagram

The execution time of this circuitry completely depends on the required number of user blocks and the number of free rectangles on the FPGA surface. In the best case, when the whole FPGA surface is empty, the region allocation takes just 9 cycles (22.5 nanoseconds). The worst case occurs if the required user block is the half of the total user blocks and if the device usage is similar to Figure 8.2. In this case, for $10 \times 10$ user block partitioning, the region allocation takes 512.5 nanoseconds. 


\subsubsection{Phase-2 Circuitry}

In this phase, the physical mapping of the user blocks given by the hardware task scheduler is performed. As was mentioned above, the user blocks having data dependency are kept in the memory as a linked list of the given node structure. Therefore, the input port of this circuitry for the hardware task scheduler is just the front node of the linked list. Note that this is a sorted linked list with descending value of total traffic demand of the user blocks. The other input is the allocated region from phase- 1 circuitry. Similarly, a linked list is used to represent the allocated region.

Depending on the type of the allocated region, two physical mapping techniques are used and hence two different circuitry are designed. Similar to other implementations, due to its fast and single clock access features the allocated region are kept in a dual port block memory and one of the ports is connected to memory controller output of the region allocation circuitry. As shown in Figure 8.4, the other port is connected to both of the physical mapping circuitries, which are the single rectangle placer and the distributed placer circuitries

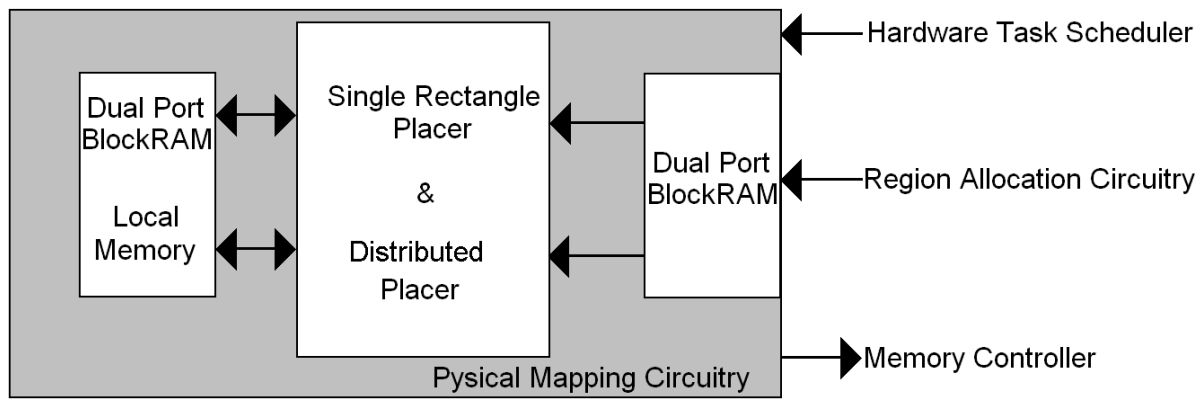

Figure 8.4: Physical mapping circuitry block diagram

Just after receiving the physical mapping request, the front node of the linked list representing the allocated region is checked by both of the circuits and if this is a single node list, only the single rectangle placer circuitry starts operation. Since mapping of user blocks depend on the Manhattan distance and the total traffic demand on the path to already placed user blocks, the communication demand between two neighboring IBCN switches is kept in local memory, which is a dual port block RAM. Upon receiving the placement request this memory is cleared 
by just applying a single cycle reset. Starting from the largest rectangle, which is at the front of the allocated region linked list and from the largest traffic demand user block, which is at the front of the user block linked list, the physical mapping is done by placing the user block to the middle of the rectangle area under consideration. Note that the placement requires just updating the Physical_location field of the user block node in the memory. Then for each user block in the linked list, the best fit placement is done by first searching for the free user block with minimum Manhattan distance to the middle of the rectangle area. If there is no free location on the current rectangle area, the user block is placed on to the middle location of the next rectangle area in the linked list. If there are more than one such free locations, the user block with IBCN switch having minimum traffic to the IBCN switch in the middle of the rectangle area is selected. Upon this selection, traffic demand of the selected user block's IBCN switch is updated.

The operation of the single rectangle placer circuitry starts by placing the rectangle onto the single user block sized virtual rectangle. Starting from the next user block in the linked list, this virtual rectangle is expanded to minimize the Manhattan distance to the placed user block with the largest traffic demand to the user block under consideration. This Manhattan distance is selected such that the virtual rectangle has width and depth to fit to the allocated region. If there are more than one alternatives for the expansion, the one with the smallest traffic demand to the largest traffic demand user block is selected. Then the traffic demand of the selected user block's IBCN switch is updated. After all user blocks are placed on the virtual rectangle, Physical_location fields of the user blocks are updated using the given free rectangle area location on the reconfigurable hardware.

Among all parts of the mapping engine, phase-2 Circuitry is the most logic block consuming one. The implementation results on Virtex6 LX760 FPGA has shown that two dual port block RAM, 6842 flip flops out of 948480 and 10832 look up tables out of 948480 is needed and the achieved operating frequency is $316 \mathrm{MHz}$. When the whole mapping engine is considered, the total logic demand is 10346 flip flops out of 948480, 17532 look up tables out of 948480 , 4 dual port block RAM out of 1440, which represents around $1.5 \%$ of the FPGA resources.

The execution time of phase- 2 circuitry completely depends on the allocated region and the number of user blocks. However, due to search on a larger number of user blocks with the same Manhattan distance and traffic demand, the single rectangle placer requires more time 
to complete. For example, the physical mapping takes 1.13 microseconds to place 25 user blocks onto an allocated region of $5 \times 5$ user blocks. This time decreases to 221 nanoseconds for the placement of 8 user blocks onto a region of $3 \times 3$ user blocks.

\subsection{System Area Circuitry Implementation Results}

In order to show the logic resource requirement of the computing platform, a complete implementation is given for the SAC. As was discussed before, the SAC is composed of the reconfigurable hardware operating system circuitry and the host\&memory interface. For this particular Virtex6 LX760 FPGA, the whole SAC implementation is depicted in Figure 8.5.

In this implementation, the IBCN access points (AP) are connected to hardware operating system over a shared bus architecture. In order not to stall this shared bus, buffers are employed in IBCN access points and its size is selected as 64 entries with 10 bits. The external memory choice for the implemented reconfigurable computing platform is a 72 bit DDR3 SRAM and 8 bits are used for error checking and correction. Since this FPGA has hard coded PCI Express IP Core, the connectivity between the host GPP is performed over the PCI Express. Finally, the internal configuration access port (ICAP) IP is used for the management of the partial reconfiguration. The resource requirements and the operating frequency of these SAC components are listed in Table 8.5.

Table 8.5: Circuit size and performance of SAC components on Xilinx Virtex6 LX760

\begin{tabular}{lll}
\hline Circuit & Logic Requirements & Performance \\
\hline HWICAP IP [74] & $\begin{array}{l}\text { Slice FF pairs : 717 out of 948480 } \\
\text { Slice LUTs : 900 out of 948480 }\end{array}$ & 32 bit @ 125MHz \\
\hline DDR3 SDRAM & Slice FF pairs : 5582 out of 948480 & 72 bit @ 1066MHz \\
Controller [75] & Slice LUTs :5899 out of 948480 & \\
\hline PCI Express IP [76] & Slice FF pairs : 500 out of 948480 & x4 lane PCI Express, \\
& Slice LUTs :625 out of 948480 & @ 2.5 / 5GHz \\
\hline IBCN access point & Slice FF pairs : 756 out of 948480 & 374 MHz \\
with 64x10 bit buffer & Slice LUTs :420 out of 948480 & \\
\hline Hardware Operating & Slice FF pairs : 14532 out of 948480 & Hardware Task \\
System & Slice LUTs :21653 out of 948480 & Scheduler @ 274Mhz \\
& Block Ram :12 out of 1440 & \\
\hline
\end{tabular}


For this particular FPGA, when the reconfigurable hardware is partitioned into $10 \times 10$ user blocks, about 5\% percent of the FPGA resources are reserved for the SAC implementation. Since the logic demand for IBCN will be $13 \%$, the remaining $82 \%$ of the logic resources are available for the user's hardware task implementation.

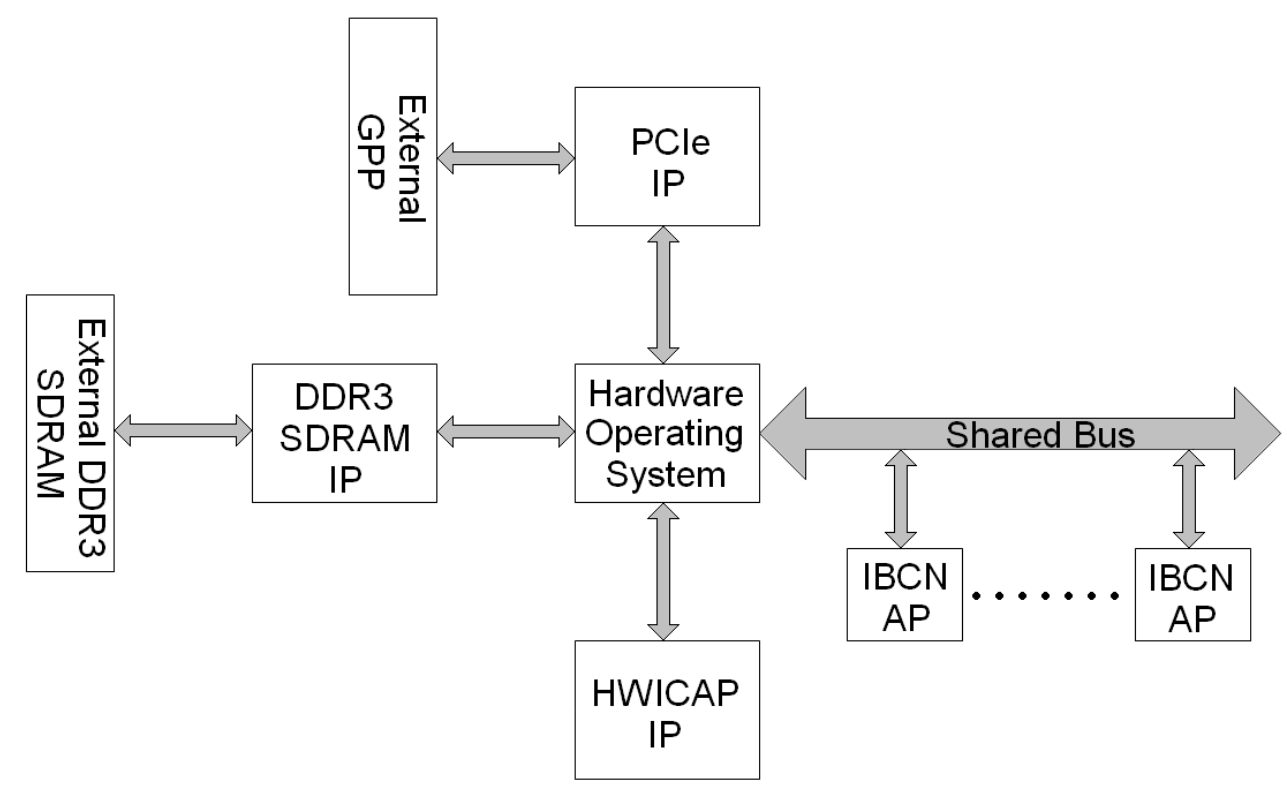

Figure 8.5: SAC implementation for Xilinx Virtex6 LX760 


\section{CHAPTER 9}

\section{COMPUTATIONAL STUDY}

Using our proposed novel reconfigurable computing platform, a substantial level of performance improvement may be achieved in terms of effective hardware task circuit size and total device utilization while providing high level of design flexibility with real time support and soft task pre-emption for priority based scheduling. In addition, the reconfiguration overhead may significantly be decreased depending on hardware task parameters. In order to illustrate these possible improvements, an extensive computational study is performed. Below, our experimental framework and simulation results are reported.

\subsection{Computational Study Results on Reconfigurable Device Utilization}

In this subsection, computational study results about reconfigurable resource management and total device utilization compared to some existing methods are reported. Among these existing methods, fixed block size approaches are applicable in embedded real time applications since others suffer from external fragmentation and require de-fragmentation. If one dimensional fixed block partitioning is employed, a shared bus is used as the communication media in general, which lowers the operating frequency of hardware tasks considerably. Therefore the comparison is made only between our reconfigurable computing platform proposal and the two dimensional fixed block size partitioning methods in [20,21].

Our computational study is carried out using a number of embedded systems that are classified according to the following parameters:

- TSR (task size ratio): ratio of the size of the largest hardware task to the smallest 
hardware task size.

- EBSR (execution block size ratio): ratio of the size of the largest execution block to the size of the smallest execution block.

- PF (parallelism factor): ratio of the number of execution blocks working in parallel to the number of execution blocks in the hardware task. It is in range $[0,1]$ and is equal to 1 if all of the execution blocks are working in parallel.

Similar to IBCN and SAC implementation, Xilinx Virtex6 LX760 FPGA is chosen as the target reconfigurable hardware. The Virtex6 LX760 series device has 118,560 slices, which is equivalent to around 5.5 million gates when CLB utilization is approximately $60 \%$.

In order to evaluate our model, we calculated $\rho_{\text {total }}$, effective_size() and improvement in $\rho_{\text {total }}$ as compared to [20,21] for different embedded system classes. Each embedded system class is classified by its $T S R, E B S R$ and $P F$ values. In total, 640 embedded system classes are studied using eight $T S R$, eight $E B S R$ and ten $P F$ values. In each embedded system class $\rho_{\text {total }}$, effective_size() and improvement in $\rho_{\text {total }}$ are calculated for 100 embedded systems each composed of 1000 randomly generated hardware tasks using a uniform distribution. In all classes, the minimum size of a hardware task is selected to be $100 \mathrm{~K}$ gates and minimum execution block size is selected as $10 \mathrm{~K}$ gates. Note that, $\rho_{\text {total }}$ and effective_size() are independent of reconfiguration time. We have observed that all our results are within $1 \%$ vicinity of the calculated and reported averages using $95 \%$ confidence interval.

The communication media used in two dimensional fixed block models in $[20,21]$ is unknown. We therefore used our IBCN implementation as the communication media in all our comparison work.

Our computations have shown that $\rho_{\text {total }}$ and effective_size() are almost independent of TSR. Among eight different values of TSR, the worst effective_size () and $\rho_{\text {total }}$ values are obtained for $T S R=1.75$ and therefore we present here the variation of these parameters against parallelism factor $P F$ and execution block ratio $E B S R$ for $T S R=1.75$ case only. In Appendix, the computational results for other $T S R$ values are reported.

Figure 9.1 shows that if the tasks are not highly parallel (having smaller $P F$ ) the hardware task circuitry can be executed on a smaller area on the reconfigurable hardware. An approximate 
reduction to $25 \%$ percent of the whole task circuit size is achieved in effective_size() when $P F=0.1$ and $E B S R=1$. The circuit size should ideally reduce to $10 \%$ in this case, however our proposal diverts from the ideal due to pre-fetching for shortest turnaround time and due to non-integer multiple size of the user block size compared to actual execution block sizes. Increasing $P F$ increases the circuit size and it becomes 1 when $P F$ is approximately 0.5 . The value of effective_size() reaches to at most 1.18 when $P F$ is higher, which means that we need a slightly larger (approximately 18\%) circuit area compared to the ideal requirement for highly parallel tasks due to fragmentation during the bin packing process.

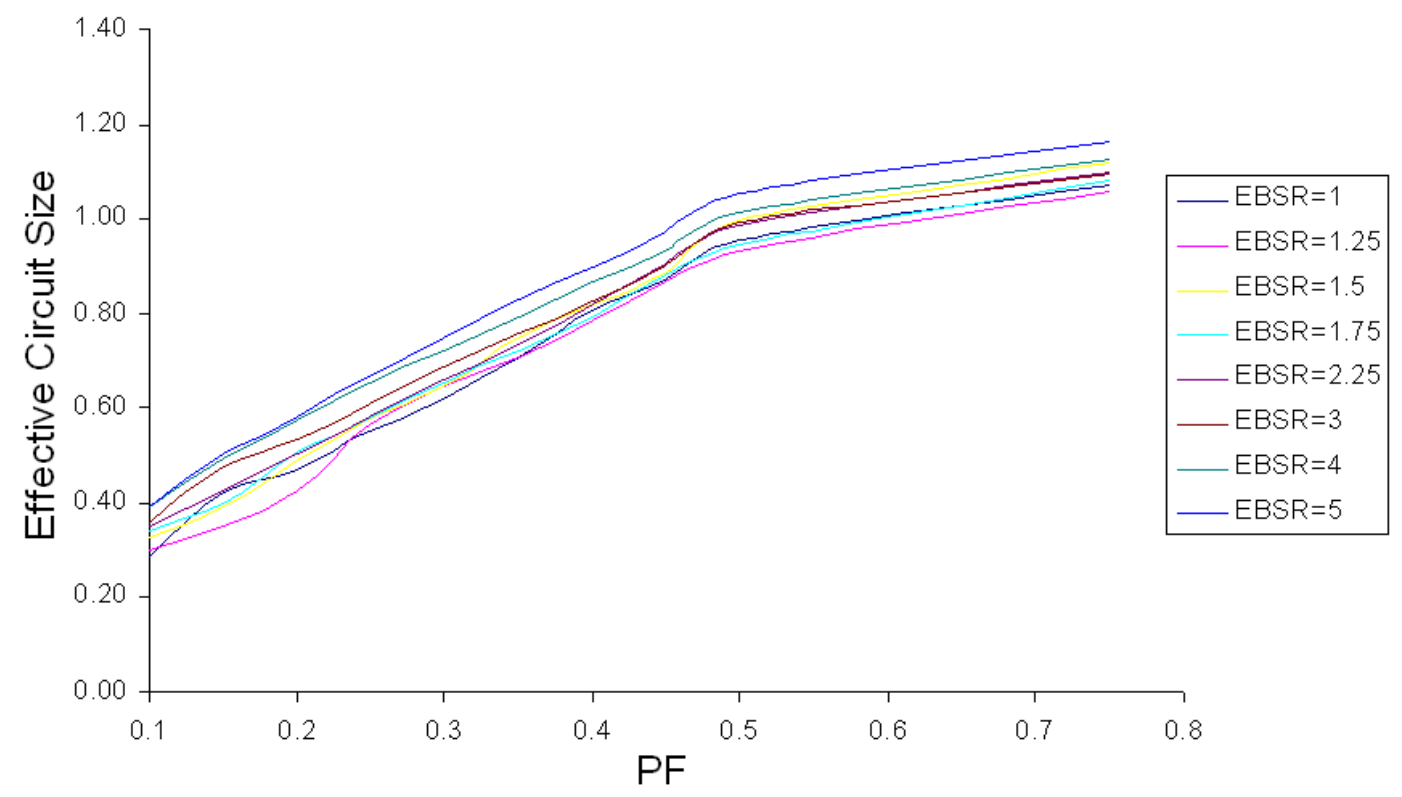

Figure 9.1: Ratio of effective_size() to whole circuit size with respect to $P F$ for $T S R=1.75$ case

For the embedded system class with $P F=1$ and $E B S R=1, \rho_{\text {total }}$ is observed to be 3.24 at most (see Figure 9.2). Since we have reduction in hardware task circuit sizes, $\rho_{\text {total }}$ is more than unity. With increasing $E B S R, \rho_{\text {total }}$ slightly decreases and reaches to 2.44 for $E B S R=5$. Similar to effective_size (), increasing $P F$ results in a decrease in $\rho_{\text {total }}$ and the minimum utilization is $79 \%$ in our computational study. Therefore, we conclude that $21 \%$ of the reconfigurable hardware resources are wasted in the worst case because of our IBCN implementation and fragmentation due to the bin packing process.

We observed that the effect of TSR is negligible for both effective_size() and $\rho_{\text {total }}$. How- 


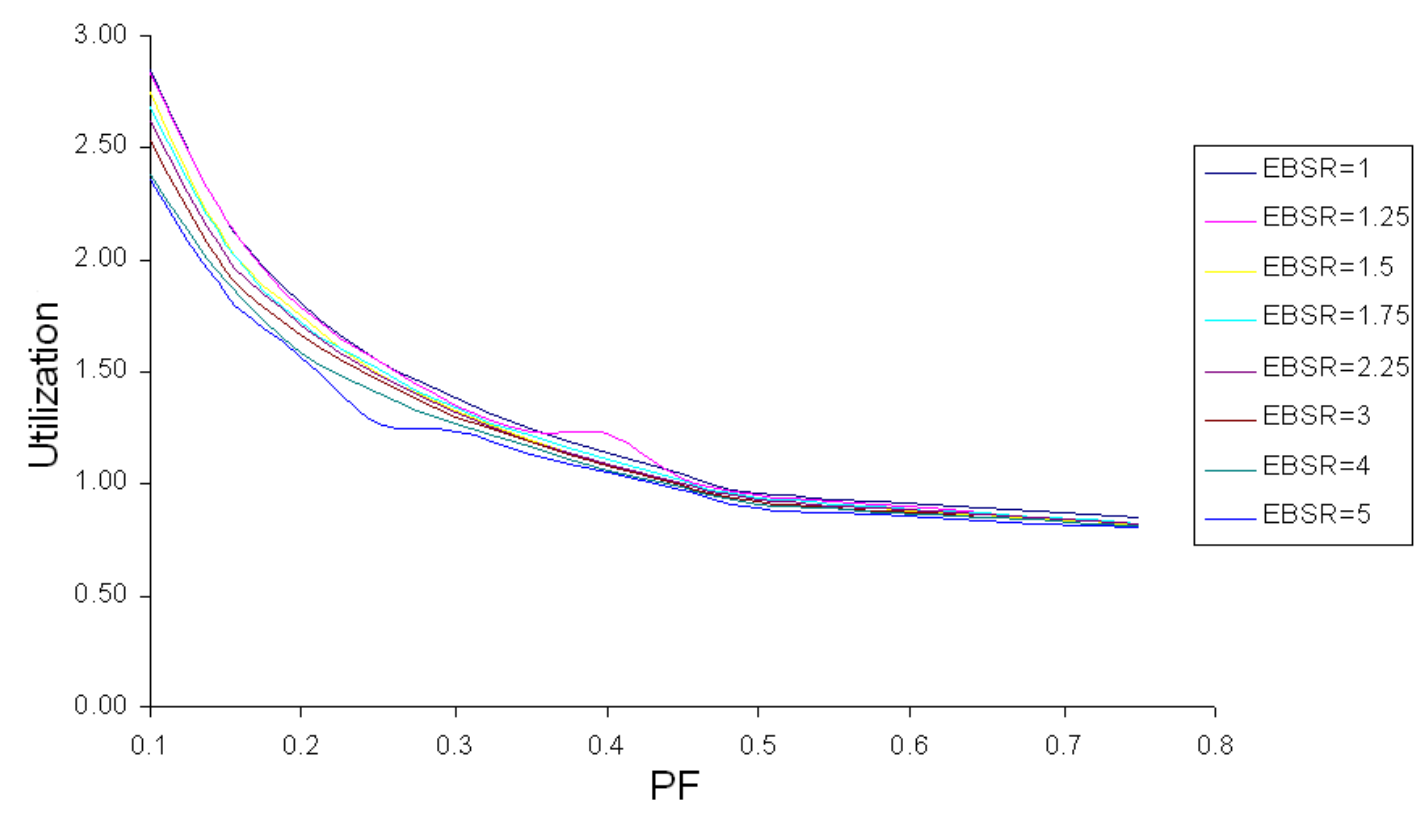

Figure 9.2: $\rho_{\text {total }}$ with respect to $P F$ for $T S R=1.75$ case

ever, this is not the case in the existing two dimensional fixed block size partitioning methods $[20,21]$. As expected, the utilization improvement as compared to existing methods is observed when the embedded system is composed of equal sized tasks, i.e. when $T S R=1$. Except the worst case when $P F \geq 0.5$ and $T S R=1$, we have achieved an improvement in $\rho_{\text {total }}$. The improvement in $\rho_{\text {total }}$ is observed to vary slightly with $E B S R$ and the worst case variation is shown in Figure 9.3. Figure 9.4 shows that $\rho_{\text {total }}$ improvement increases with decreasing $P F$ and increasing $T S R$ and $11.64 \times \rho_{\text {total }}$ improvement is achieved in $P F=0.1$, $E B S R=1$ and $T S R=5$ case.

\subsection{Computational Study Results on Reconfiguration Overhead}

Thanks to partitioning of hardware tasks into a set of execution blocks, the reconfiguration overhead can be reduced significantly. This improvement depends on the spatial and temporal partitioning characteristics of the hardware tasks and the execution time to reconfiguration time ratio (ET2RTR) of execution blocks.

In order to illustrate the effect of these factors, for each $P F$ value and execution time to reconfiguration time ratio pair we have simulated 100 embedded systems each composed of 


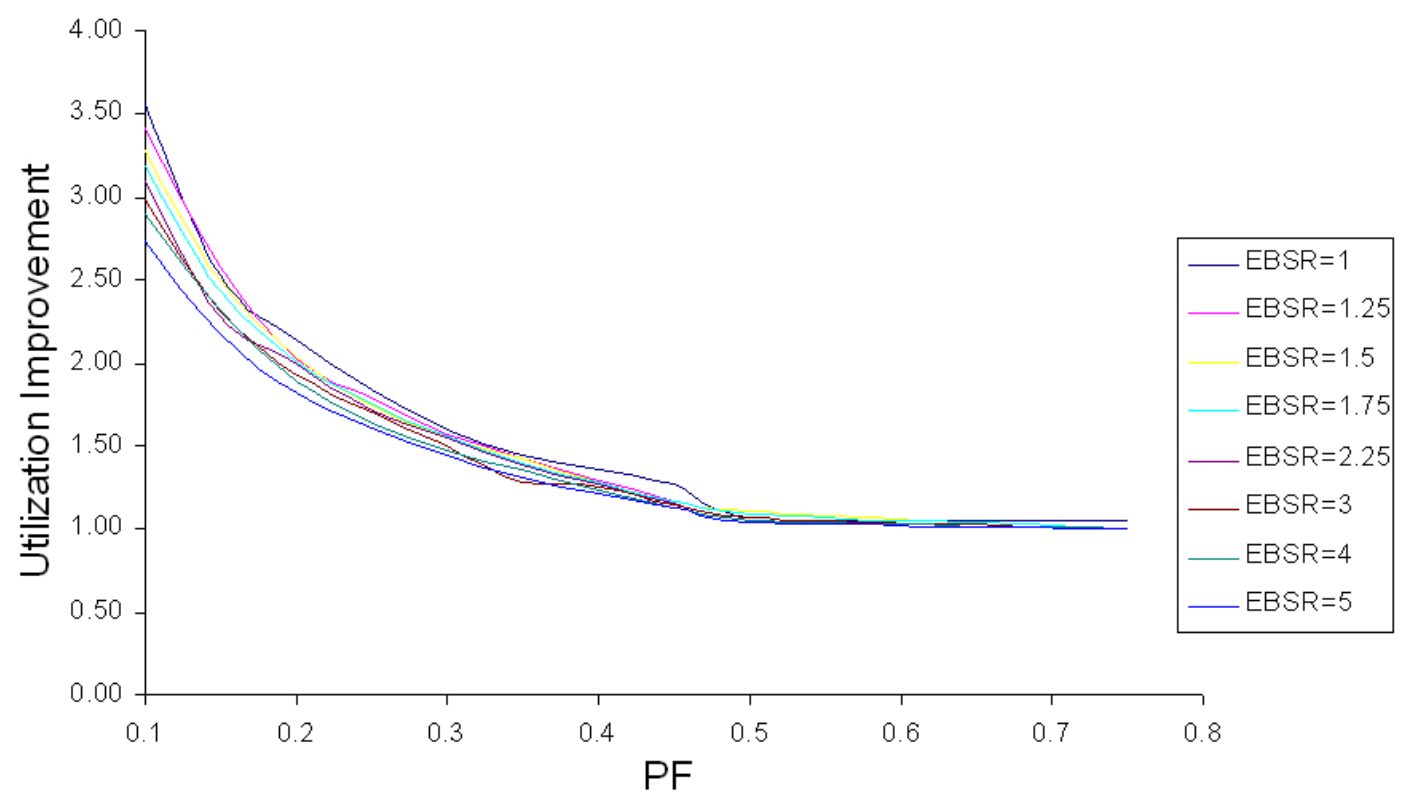

Figure 9.3: Ratio of $\rho_{\text {total }}$ with our proposal to that of 2D fixed partitioning [20,21] against $P F$ for $T S R=1$

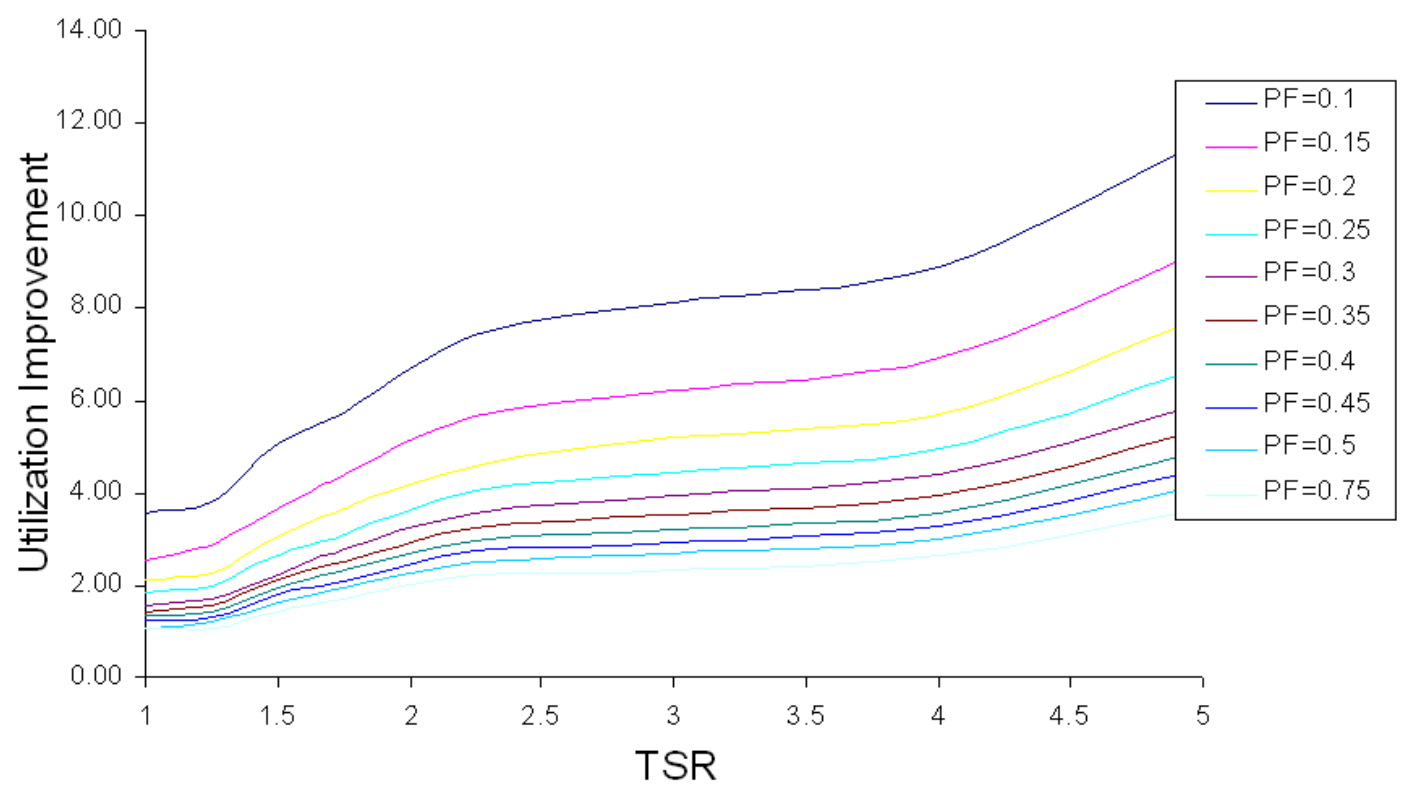

Figure 9.4: Ratio of $\rho_{\text {total }}$ with our proposal to that of 2D fixed partitioning [20,21] against $T S R$ for $E B S R=1$ 
1000 hardware tasks. The number of execution blocks for a task is randomly selected between 100 and 200 in a uniformly distributed manner. In a similar fashion, the execution block size is randomly selected to be between $10 \mathrm{~K}$ and $40 \mathrm{~K}$ gates. The device under consideration for this computational study is also Virtex6 LX760 FPGA.

Figure 9.5 presents the average achieved reconfiguration overhead in terms of percentage of reconfiguration time to total task turnaround time.

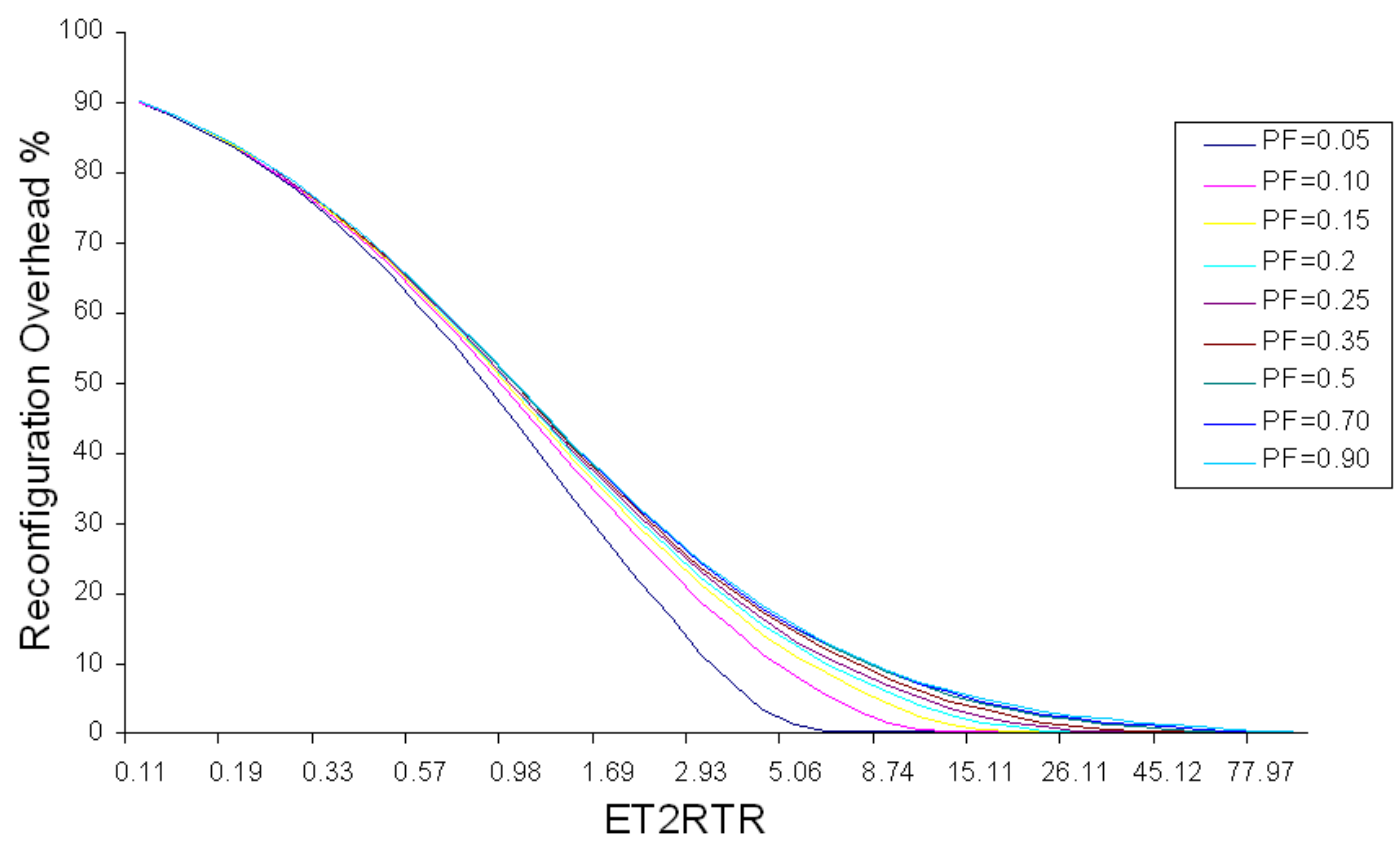

Figure 9.5: Reconfiguration overhead as percentage of total task turn around time variation against ET2RTR for different values of $P F$

The achieved reconfiguration overhead is presented in an alternative form as the percentage of reconfiguration overhead without task partitioning in Figure 9.6. As expected, a great improvement is achieved in reconfiguration overhead for hardware tasks with small PF values. Even for highly parallel tasks, for which $P F$ is greater than 0.5 , a certain level of improvement is still possible. 


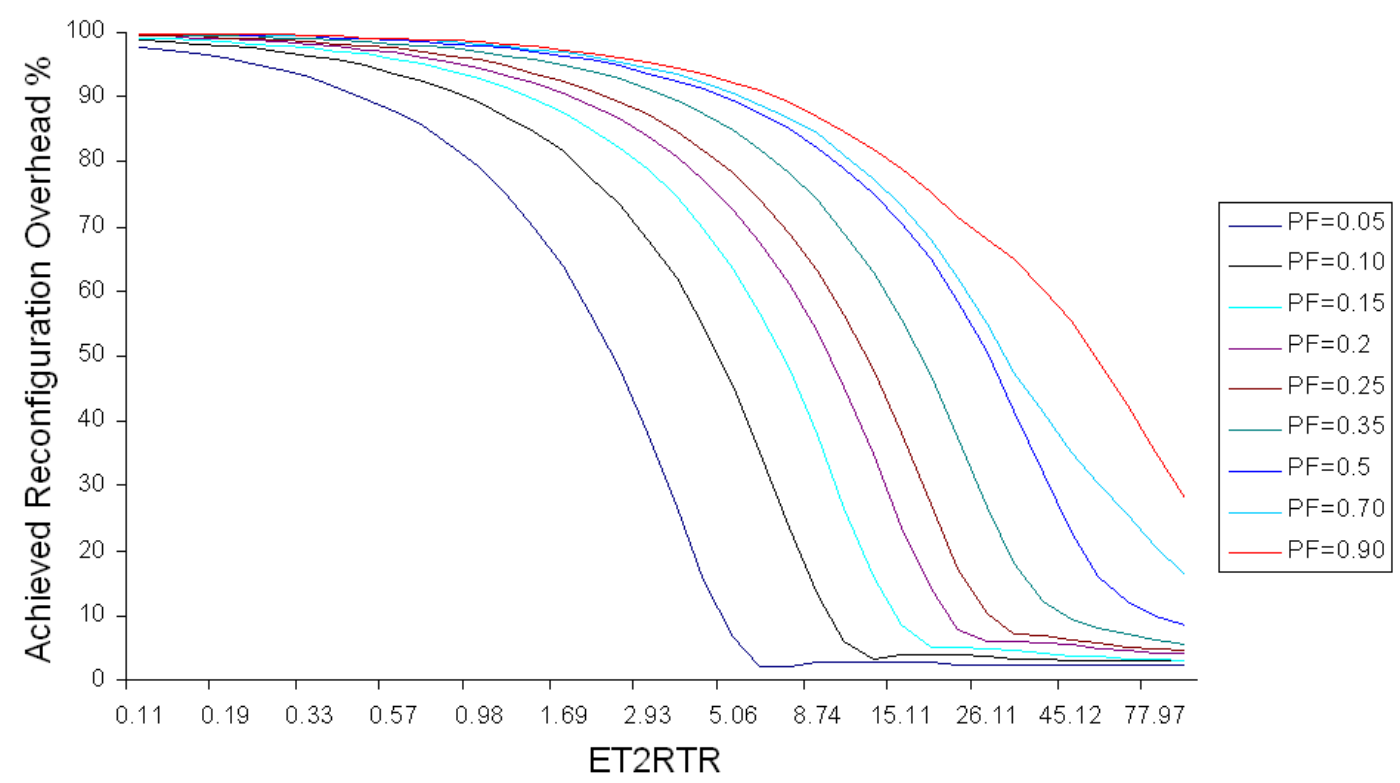

Figure 9.6: Achieved reconfiguration overhead as percentage of reconfiguration overhead without task partitioning versus ET2RTR for different values of $P F$

\subsection{Comparison Results on Device Utilization and Reconfiguration Overhead}

As was stated earlier, the idea of partitioning a hardware task into set of sub-tasks with the aim of minimizing the reconfiguration time overhead was also used by Resano et al. [26,27]. While providing a higher device utilization our reconfigurable computing platform may have a higher reconfiguration overhead as compared to $[26,27]$ due to our packing process.

A comparative study on the test set used previous in the section is performed and the results are presented in Figures 9.7 and 9.8. As shown in Figure 9.7, with increasing execution time to reconfiguration time ratio, the reconfiguration overhead is increased and reaches to $4 \times$ compared to $[26,27]$. However, for a large value of execution time to reconfiguration time ratio, this drawback is still acceptable because the corresponding reconfiguration overhead, which can be deduced using Figure 9.5, is very small. Despite such a small reconfiguration overhead increase, as depicted in Figure 9.8 our method provides around 1.6× utilization improvement as compared to $[26,27]$. 


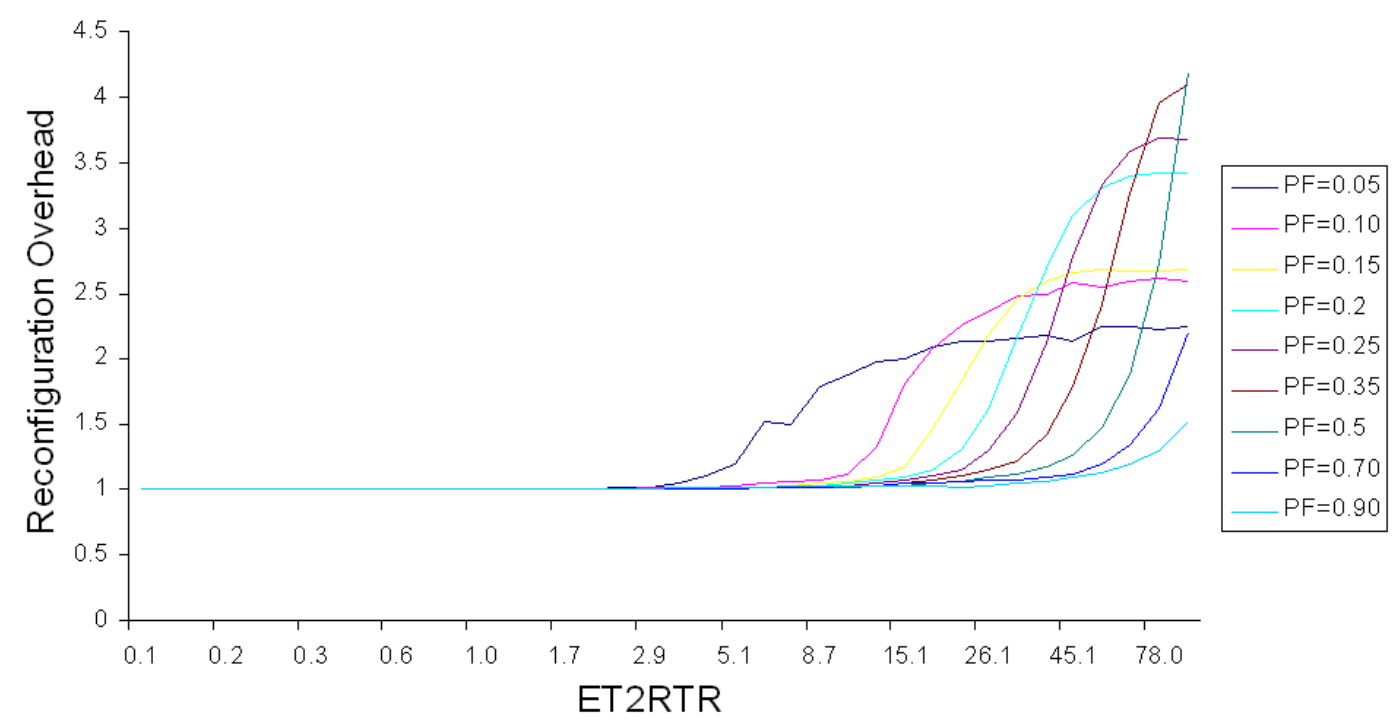

Figure 9.7: Ratio of reconfiguration overhead our proposal to reconfiguration overhead with [26,27] against $P F$

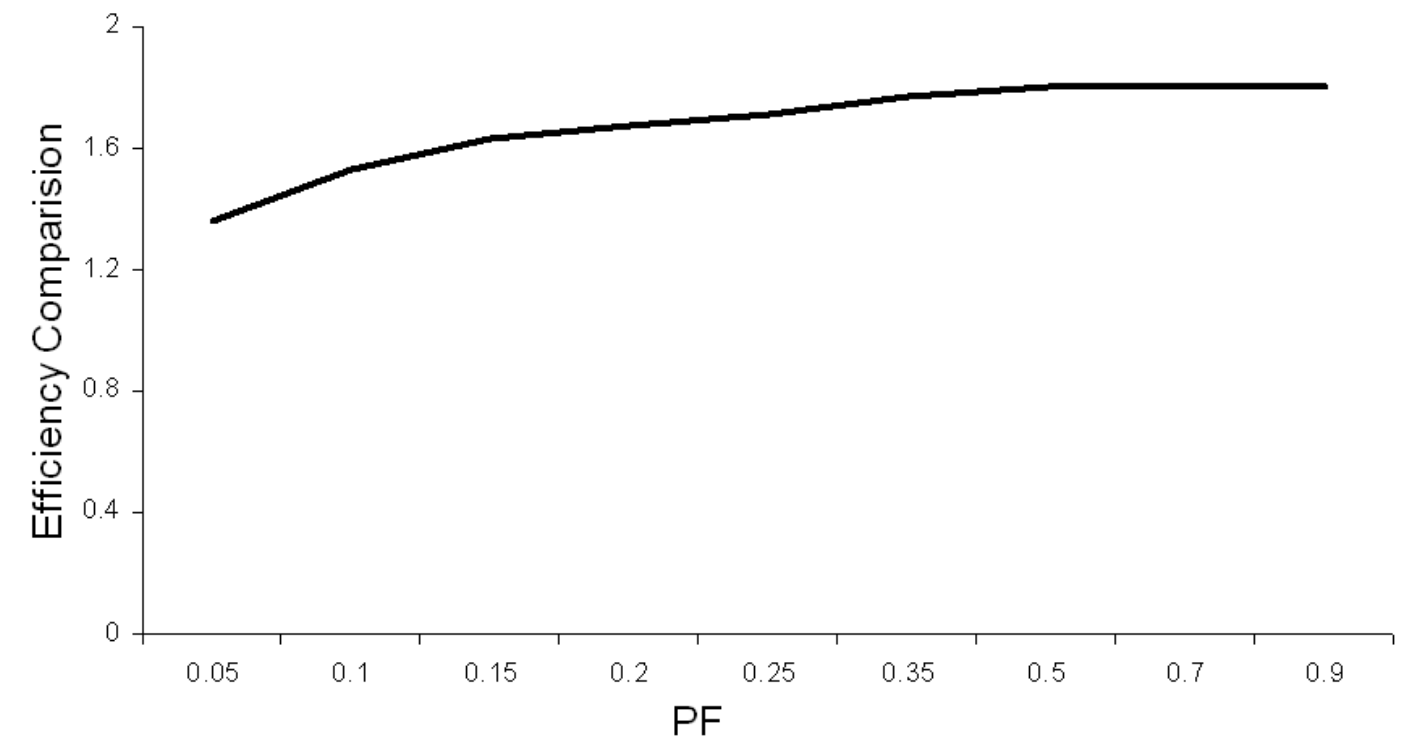

Figure 9.8: Ratio of $\rho_{\text {total }}$ with our proposal to that of [26,27] against $P F$ 


\section{CHAPTER 10}

\section{APPLICATION EXAMPLE}

A candidate application is chosen our complete reconfigurable computing platform solution.

This application is an image and sensor fusion application for an airborne defense system. As depicted in Figure 10.1, four thermal-CCTV camera pairs mounted on turret and an external airborne defense radar are used to detect and track airborne threats such as aircraft fighters, helicopters and missiles.

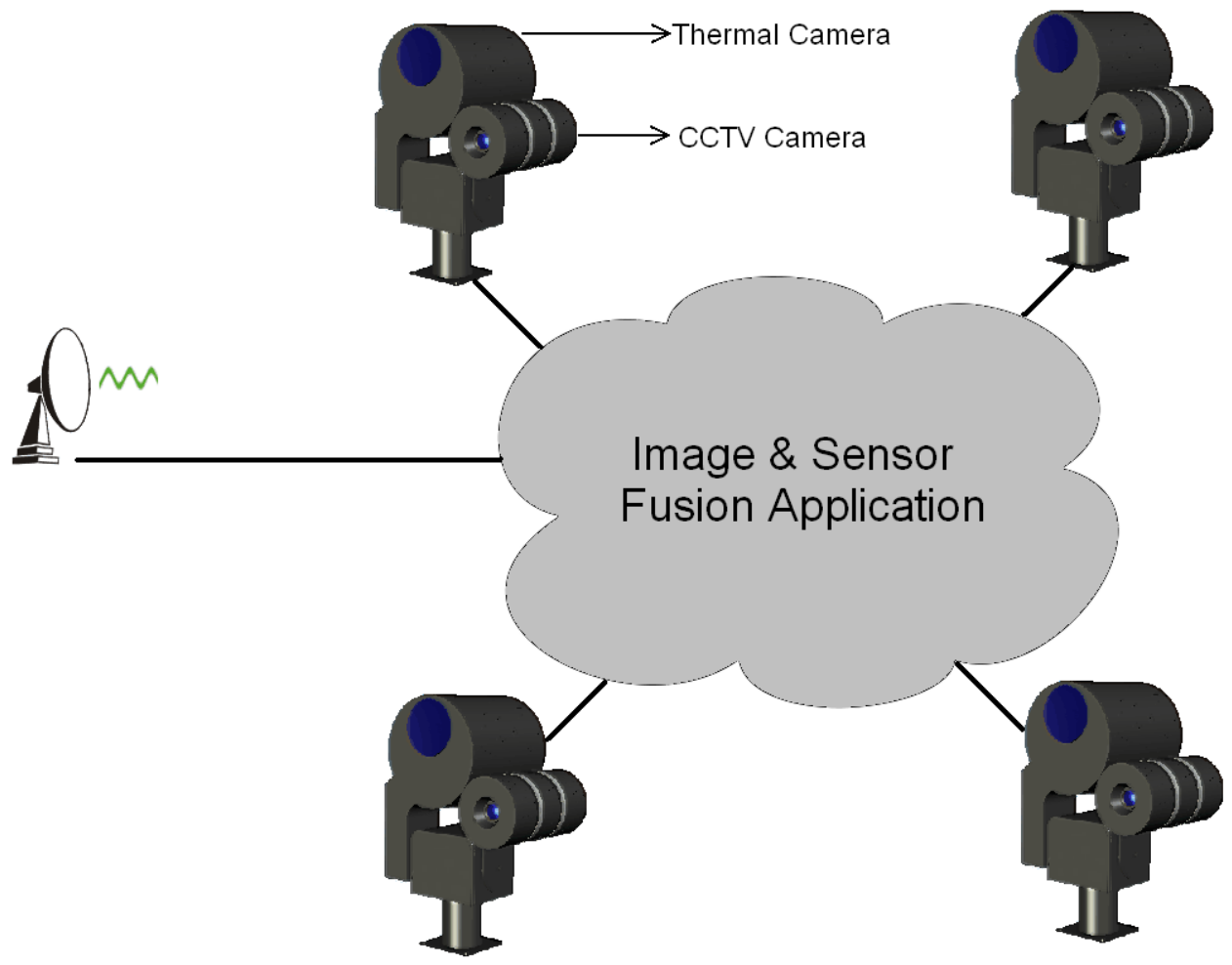

Figure 10.1: Application example: Image and sensor fusion to detect and track airborne threats 
In addition to running fusion, detection and tracking algorithms, the computing platform in this application has the following functionalities. Airborne defense radar provides its output over a radio network in JPEG2000 compressed format due to its low latency requirement, due to the need for resilience against errors and due to keeping a balance between compression and quality. Hence in this application, the computing platform performs JPEG2000 decoding of the radar data. The video received from thermal and CCTV camera pairs are compressed (encoded) in MPEG-4 format for storage. In the test mode or training mode, instead of using live video from thermal and CCTV camera pairs, this locally stored video in MPEG-4 format is then decoded. Finally, the detection and tracking information is sent in JPEG2000 format over a radio link to the information center.

For the system described above, our complete reconfigurable computing platform solution proposal is a good candidate due to its support for true multitasking, its design flexibility, short turnaround time and efficient device utilization. As shown in Figure 10.2, a possible underlying hardware is composed of a PowerPC based dual core processor MPC8640D, a Xilinx Spartan6 series FPGA used for glue logic and connectivity and a Xilinx Virtex6 LX760 FPGA used as the actual reconfigurable hardware resource.

The system operates as follows: A synchronizer in Spartan3 FPGA sends a trigger signal to the application software running on PowerPC. Depending on the source selection, the application software initially schedules MPEG-4 encoders or decoders and JPEG2000 decoder to Virtex6 FPGA. When frame decoding is completed, the application software schedules;

- a fusion algorithm selected among four fusion algorithms based on availability and latency of radar information and environmental conditions such as fog, rain, snow, day and night,

- two different target detection algorithms selected among five detection algorithms based on environmental conditions and detection information of the previous frame and

- up to four instances of tracking algorithms selected among three tracking algorithms based on the number of tracked targets and target types.

Note that up to seven tasks are scheduled to operate simultaneously. Tracking and detection algorithms process the output of the fusion algorithm of the previous stage. With the completion of these tasks, the application software is then notified and the JPEG2000 encoding of 


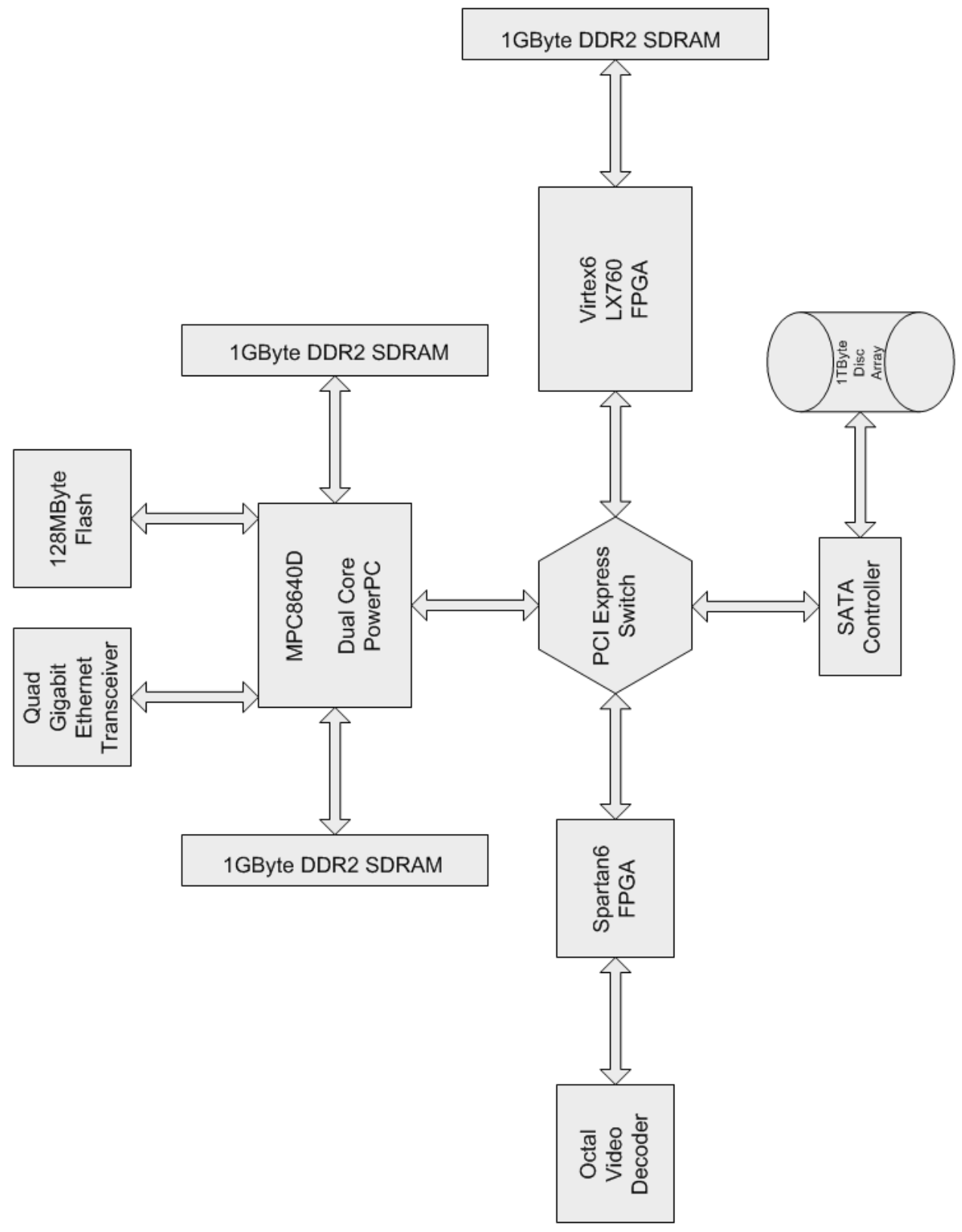

Figure 10.2: Complete reconfigurable computing solution for the application example 
the image with a track symbol on tracked targets is initiated. Finally, the application software is informed about the completion of JPEG2000 encoding whenever it happens.

The circuit parameters for MPEG-4 encoder and decoder are estimated by mapping already reported results in the literature to our target FPGA. The circuit for fusion, detection and tracking are obtained by converting the MATLAB codes to VHDL. The circuit sizes of the algorithms are obtained after synthesis and placement/routing with Xilinx ISE tools and the execution times are calculated using post timing analysis. Similarly, circuit sizes and execution times for JPEG2000 encoder and decoder are obtained by re-synthesizing an already existing design for Virtex-6. The circuit size, execution time, reconfiguration time, effective circuit size and achieved reconfiguration time of each task is listed in Table 10.1.

Table 10.1: Parameters of individual hardware tasks in the application example

\begin{tabular}{llllll}
\hline $\begin{array}{l}\text { Task } \\
\text { Name }\end{array}$ & $\begin{array}{l}\text { Circuit } \\
\text { Size } \\
\text { (slice) }\end{array}$ & $\begin{array}{l}\text { Execution } \\
\text { Time } \\
\text { (msec) }\end{array}$ & $\begin{array}{l}\text { Reconfiguration } \\
\text { Time } \\
\text { (msec) }\end{array}$ & $\begin{array}{l}\text { Effective } \\
\text { Circuit } \\
\text { Size } \\
\text { (slice) }\end{array}$ & $\begin{array}{l}\text { Achieved } \\
\text { Reconfiguration } \\
\text { Time } \\
(\mathbf{m s e c})\end{array}$ \\
\hline $\begin{array}{l}\text { MPEG-4 } \\
\text { Decoder [77] }\end{array}$ & 4742 & 2.34 & 1.749 & 1440 & 0.342 \\
MPEG-4 & 8622 & 4.81 & 3.181 & 4320 & 0.719 \\
Encoder [78] & & & & & \\
JPEG2000 & 5256 & 2.62 & 1.941 & 2880 & 0.31 \\
Decoder & & & & & \\
JPEG2000 & 6049 & 3.25 & 2.232 & 2880 & 0.468 \\
Encoder & & & & & \\
Fusion-1 & 27106 & 14.3 & 10.002 & 12960 & 2.543 \\
Fusion-2 & 14203 & 16.1 & 5.241 & 7920 & 0.692 \\
Fusion-3 & 19932 & 11.7 & 7.355 & 8640 & 1.345 \\
Fusion-4 & 23335 & 14.2 & 8.611 & 12240 & 2.576 \\
Detection-1 & 24552 & 15.1 & 9.06 & 12240 & 1.094 \\
Detection-2 & 17620 & 12 & 6.502 & 10800 & 1.272 \\
Detection-3 & 19652 & 14.2 & 7.252 & 8640 & 1.338 \\
Detection-4 & 23299 & 12.7 & 8.597 & 12960 & 1.17 \\
Detection-5 & 18363 & 15.3 & 6.776 & 10080 & 1.579 \\
Tracking-1 & 10753 & 5.5 & 3.968 & 2880 & 0.763 \\
Tracking-2 & 13340 & 6.1 & 4.922 & 7920 & 0.856 \\
Tracking-3 & 11304 & 5.8 & 4.171 & 7200 & 0.905 \\
\hline
\end{tabular}

Note that the in Table 10.1, circuit size is the number of FPGA slices needed to implement 
the associated hardware task as a single circuit. Similarly, the reconfiguration time is the time needed to load the whole task circuitry without any partitioning. We then calculated, for this particular FPGA, the effective circuit size, which represents the maximum area needed at any instance for the task to be executed. Also the achieved reconfiguration overhead is calculated taking the partial reconfiguration time and the mapping decision time into consideration.

For this application, our user block size selection heuristic has found that the FPGA surface should be partitioned in $12 \times 12$ user blocks each having a size of 720 slices in the shape of $40 C L B \times 9 C L B$ and the device utilization has turned out to be 1.85 . Without using the proposed reconfigurable computing platform, the application example, described above, needs $551 \mathrm{~K}$ slices and will have a maximum frame processing time of 24.16 milliseconds in the worst case. Thanks to partial reconfigurability concept and our novel platform proposal that the resource demand is reduced to $69 \mathrm{~K}$ slices and the worst case processing time is just increased to 28.59 milliseconds which means that 30 frames per second processing speed can be used. This time includes the execution time, mapping decision and reconfiguration time of the user blocks. Note that, the time overhead due to IBCN communication and the shared memory access is not taken into account as it requires a very complicated simulation study. 


\section{CHAPTER 11}

\section{CONCLUSIONS AND FUTURE WORK}

In this chapter, conclusions of this thesis work are stated. A brief discussion on the contributions made by this dissertation is given. In addition, a discussion on the future work to improve and extend the application area of the presented reconfigurable computing platform is presented.

\subsection{Contributions}

In this thesis work, we present a complete reconfigurable computing platform for embedded applications. This reconfigurable computing platform is developed to give design flexibility similar to software development for conventional computing with general purpose processors. The proposed reconfigurable computing platform is a combination of a universal reconfigurable hardware, which is very similar to commercial FPGA devices, and a general purpose processor. The application programmer is allowed to design the applications with both hardware and software tasks. With a specially designed operating system architecture, the details of the underlying hardware is hidden from the application programmer and high level operating system services are provided to both software and hardware tasks. In addition, with its deterministic response time, our reconfigurable computing platform proposal is well suited for embedded applications with real time requirements.

In our reconfigurable computing platform, the application programmer is responsible to give the hardware task only. For this purpose, we have introduced a novel hardware tasks model and this task model requires a hardware task to be partitioned by the application programmer into sub tasks, which is called execution blocks, using both temporal and spatial par- 
titioning processes. Thanks to this novel hardware task model, our proposal requires less reconfigurable hardware area for implementing the circuitry of the hardware task and the reconfiguration time overhead is kept at low level, which results in short task turnaround time. In addition, by starting and stopping hardware tasks at this execution block boundaries, our reconfigurable computing platform supports preemption for priority based scheduling.

In the proposed reconfigurable computing platform the reconfigurable hardware is completely managed by the hardware operating system and a novel two dimensional surface partition process is used. The proposed partitioning technique allocates fixed size regions for hardware task implementation and provides an interconnection media for both external memory access and communication between fixed size regions. With this interconnection media, the execution blocks of hardware tasks can be placed onto non-contiguous areas on the reconfigurable hardware and hence the whole reconfigurable hardware surface can be used in an efficient manner.

It has been observed that the surface partitioning parameters determine the reconfigurable hardware utilization and hence a mathematical model for selecting the the surface partitioning parameters is given. A greedy heuristic technique employing bin packing process is used to solve this problem and it has been shown that the gap between the results of our greedy heuristic and the relaxed upper bound is just $4.5 \%$.

As an interconnection media, a special two dimensional mesh NoC architecture with wormhole routing is designed. For this purpose a special wormhole switch architecture is developed and its implementation results has shown that a very high bandwidth can be offered with low logic demand. For Xilinx Virtex6 LX760, when the FPGA surface is partitioned into $10 \times 10$ fixed blocks, only $13 \%$ of the logic resources are used for this NoC implementation and 62.4 GByte/s aggregate bandwidth is achieved.

The performance of reconfigurable computing platform proposal has been evaluated with an extensive computational study in terms of reconfigurable hardware resource management and reconfiguration time overhead. It has been shown that our solution has superior performance in both reconfigurable hardware utilization and reconfiguration time overhead but of course depending on the embedded system parameters. When compared to existing two dimensional surface partitioning techniques [20,21], in which the hardware task is loaded onto a contiguous area, depending on the sizes of the hardware task and the number of execution blocks 
required to work in parallel up to 11.64 times improvement is achieved in reconfigurable hardware utilization. Similarly, depending on the number of execution blocks required to work in parallel, the execution time and the circuit size, the reconfiguration time overhead is decreased up to $3 \%$ of task execution time. When compared to existing techniques [26,27], in which the hardware tasks are partitioned into fixed size sub-tasks, our computing platform achieves a bit larger reconfiguration time overhead. However, the reconfigurable hardware utilization in our proposal is around 1.6 times that of $[26,27]$.

In our reconfigurable computing platform proposal, application programmer is responsible to partition his/her hardware task into execution blocks. The partitioning process must be done considering both temporal and spatial partitioning and it is allowed to have execution blocks with data dependency. Since this data dependency is handled by our NoC, the execution time of the hardware task will be increased due to the NoC's latency. Hence it is not recommended to have a partition with high rate data dependency because such partitioning may result in unacceptable increase in the execution time. In case such a partitioning still occurs, we propose, as a remedy, an online two phase ad-hoc mapping technique that finds suitable locations for the execution blocks to keep the communication overhead on the $\mathrm{NoC}$ as low as possible. The proposed ad-hoc solution is a multi objective mapping technique, which tries to minimize the store and forward latency, minimize path blocking due to wormhole routing and balance external memory access.

Finally, a real life application, which is an image and sensor fusion application to detect and track airborne threats, is solved using our reconfigurable computing platform. For this specific application, the reconfigurable hardware resource requirement is reduced to $14 \%$ of the original resource requirement with just a $19 \%$ increase in execution time.

\subsection{Future Work}

In the proposed reconfigurable computing platform, the application programmer responsibility is only to partition the application into a set of execution blocks. This partitioning process affects both device utilization and task turn around time. In addition, the IBCN communication latency increases if the execution blocks have too much data dependency. As future work, a partitioning technique can be developed so that the application programmer is just respon- 
sible to write the application in a hardware description language. This partitioning technique will take both the temporal and spatial behaviors of the given task to generate the execution blocks to maximize the computing platform performance.

Within the scope of this thesis work, the hardware task scheduler and the associated components are designed to support the scheduling of hardware tasks in any order and for unknown arrival times. In some embedded applications, it is possible to know the scheduling sequence and arrival times. Therefore, it is possible to improve the task turn around time and hence the system performance with an offline optimization process. As future work, an offline mapping and scheduling technique can be developed to find the physical location and mapping of the user blocks that minimizes the embedded system's response time. 


\section{REFERENCES}

[1] G. Estrin, Organization of computer systems-the fixed plus variable structure computer, Proc. of Western Joint Computer Conference, (1960) 33-40.

[2] G. Lu, M. Lee, H. Singh, N. Bagherzadeh, F. Kurdahi, E. Filho, MorphoSys: a reconfigurable processor targeted to high performance image application, Proc. 6th Reconfigurable Architectures Workshop RAW, (1999) 661-669.

[3] X. Tang, M. Aalsma, R. Jou, A compiler directed approach to hiding configuration latency in chameleon processors, International Conference on Field Programmable Logic and Applications FPL, (2000) 29-38.

[4] S.R. Alam, P.K. Agarwal, M.C. Smith, J.S. Vetter, D. Caliga, Using FPGA devices to accelerate biomolecular simulations, Computer, vol. 40. No. 3, (2007) 66-73.

[5] E. Grayver, B. Daneshrad, A reconfigurable 8 GOP ASIC architecture for high-speed data communications, IEEE Journal on Selected Areas in Communications, vol. 18, no. 11, (2000) 2161-2171.

[6] R.W. Duren, R.J. Marks, P.D. Reynolds, M.L. Trumbo, Real-time neural network inversion on the SRC-6e reconfigurable computer, IEEE Transactions on Neural Networks, vol. 18, no. 3, (2007) 889-901.

[7] M.D. Galanis, G. Dimitroulakos, C.E. Goutis, Speedups and energy reductions from mapping DSP applications on an embedded reconfigurable system, IEEE Transactions on Very Large Scale Integration (VLSI) Systems, vol. 15, no. 12, (2007) 1362-1366.

[8] Cray Inc., http://www.cray.com/, last visited on August 2011.

[9] SGI Inc., http://www.sgi.com/, last visited on August 2011.

[10] Q. Deng, S. Wei, H. Xu, Y. Han, G. Yu, A reconfigurable RTOS with HW/SW coscheduling for SOPC, Second International Conference on Embedded Software and Systems, (2005) 1-6

[11] H. Walder, M. Platzner, Non-preemptive multitasking on FPGAs task placement and footprint transform, Proc. of Int. Conf. on Engineering of Reconfigurable Systems and Algorithms, (2002) 24-30.

[12] K. Bazargan, R. Kastner, M. Sarrafzadeh, Fast template placement for reconfigurable computing systems, IEEE Design and Test of Computers, (2000) 68-83.

[13] H. Walder, C. Steiger, M. Platzner, Fast online task placement on FPGAs: free space partitioning and 2D-hashing, Proc. of Int. Parallel and Distributed Processing Symposium, (2003) 178-185. 
[14] Z. Gu, W. Liu, J. Xu, J. Cui, X. He, Q. Deng, Efficient algorithms for 2D area management and online task placement on runtime reconfigurable FPGAs, Microprocessors and Microsystems, vol. 33, no. 5-6, (2009) 374-387.

[15] M. Handa, R. Vemuri, An efficient algorithm for finding empty space for online FPGA placement,Design Automation Conference, (2004) 960-965.

[16] O. Diessel, H. ElGindy, M. Middendorf, H. Schmeck, B. Schmidt, Dynamic scheduling of tasks on partially reconfigurable FPGAs, IEE Proceedings on Computers and Digital Techniques, vol. 147, no. 3, (2002) 181-188.

[17] G. Brebner, O. Diessel, Chip-based reconfigurable task management, Lecture Notes in Computer Science, Springer, vol. 2147, (2001), 182-191.

[18] H. Walder, M. Platzner, Online scheduling for block-partitioned reconfigurable hardware, in Proc. of the IEEE Design, Automation and Test in Europe Conference and Exhibition, (2003) 290-295.

[19] H. Walder, M. Platzner, A runtime environment for reconfigurable hardware operating systems, Lecture Notes in Computer Science, Springer, vol. 3203, (2004) 831-835.

[20] T. Marescaux, A. Bartic, V. Dideriek, S. Vernalde, R. Lauwereins, Interconnection networks enable fine-grain dynamic multi-tasking on FPGAs, Proc. of 12th Int. Conf. on Field-Programmable Logic and Applications, (2002) 795-805.

[21] P. Merino, M. Jacome, J.C. Lopez, A methodology for task based partitioning and scheduling of dynamically reconfigurable systems, Proc. of IEEE Symp. on FPGAs for Custom Computing Machines, (1998) 324-325.

[22] K. Compton, L. Zhiyuan, J. Cooley, S. Knol, S. Hauck, Configuration relocation and defragmentation for run-time reconfigurable computing, IEEE Transactions on Very Large Scale Integration (VLSI) Systems, vol. 10, no. 3, (2002) 209-220.

[23] M.J. Wirlhlin, B.L. Hutchings, A dynamic instruction set computer, Proc. of IEEE Symp. on FPGAs for Custom Computing Machines, (1995) 99-107.

[24] S. M. Scalera, J. R. Vazquez, The design and implementation of a context switching FPGA, IEEE Symposium on Field-Programmable Custom Computing Machines, (1998) 78-85.

[25] L. Bauer, M. Shafique, J. Henkel, A computation and communication infrastructure for modular special instructions in a dynamically reconfigurable processor, Proc. of Int. Conference on Field Programmable Logic and Applications, (2008) 203-208.

[26] J. Resano, D. Verkest, D. Mozos, S. Vernalde, F. Catthoor, A hybrid design-time runtime scheduling flow to minimize the reconfiguration overhead of FPGAs, Microprocessors and Microsystems, vol. 28, no. 5-6, (2004) 291-301.

[27] J. Resano, D. Mozos, D. Verkest, F. Catthoor, A reconfiguration manager for dynamically reconfigurable hardware of FPGAs, IEEE Design Test of Computers, vol. 22, no. $5,(2005)$ 452-460.

[28] G.J. Brebner, A virtual hardware operating system for the Xilinx XC6200, in R. W. Hartenstein and M. Glesner, Eds. Lecture Notes in Computer Science, Springer-Verlag, vol. 1142, (1997) 327-336. 
[29] G.B. Wigley, D.A. Kearney, D. Warren, Introducing ReConfigME: an operating system for reconfigurable computing, Lecture Notes in Computer Science, Springer, vol. 2438, (2002) 687-697.

[30] M. Götz, A. Rettberg, C.E. Pereira, A run-time partitioning algorithm for RTOS on reconfigurable hardware, Lecture Notes in Computer Science, Springer, vol. 3824, (2005) 469-478.

[31] M. Edwards, P. Green, Run-time support for dynamically reconfigurable computing systems, Journal of Systems Architecture, vol. 49, no. 4-6, (2003) 267-281.

[32] K. Danne, R. Muhlenberg, M. Platzner, Executing hardware tasks on dynamically reconfigurable hardware under real-time conditions, Proc. of Int. Conference on Field Programmable Logic and Applications, (2006) 1-6.

[33] M. Ullmann, M. Hubner, B. Grimm, J. Becker, On-demand FPGA run-time system for dynamical reconfiguration with adaptive priorities, Lecture Notes in Computer Science, Springer, vol. 3203, (2004) 454-463.

[34] H. Kwork, H. So, R. Brodersan, A unified hardware software runtime environment for FPGA based reconfigurable computers using BORPH, ACM Transactions on Embedded Computing Systems, vol. 7, no. 2, (2008) 1-28.

[35] G.B. Wigley, D.A. Kearney, Research issues in operating systems for reconfigurable computing, in: Proc. of the Int. Conference on Engineering of Reconfigurable Systems and Algorithms, (2002) 10-16.

[36] Xilinx MicroBlaze soft processor core, http://www.xilinx.com/tools/ microblaze.htm, last visited on August 2011.

[37] Altera Nios II processor, http://wWw.altera.com/devices/processor/nios2/ ni2-index.html, last visited on August 2011.

[38] Xilinx Virtex-5 series FPGA, http://www.xilinx.com/support/documentation/ virtex-5.htm, last visited on August 2011.

[39] Wind River VxWorks RTOS, http://www.windriver.com/products/vxworks/ last visited on August 2011.

[40] Wind River RTLinuxFree, http://www.rtlinuxfree.com/, last visited on August 2011.

[41] Altera Stratix IV FPGA ALM Logic Structure's 8-Input Fracturable LUT, http://www.altera.com/products/devices/stratix-fpgas/stratix-iv/ overview/architecture/stxiv-alm-logic-structure.html, last visited on August 2011.

[42] Xilinx Virtex-4 series FPGA, http://www.xilinx.com/support/documentation/ virtex-4.htm, last visited on August 2011.

[43] Xilinx Partial Reconfiguration User Guide, http://www.xilinx.com/support/ documentation/sw_manuals/xilinx12_1/ug702.pdf, last visited on August 2011. 
[44] E.G. Coffman, M.R. Garey, D.S. Johnson, An application of bin-packing to multimachine scheduling, SIAM Journal of Computing, vol. 7, no. 1, (1978) 1-17.

[45] N.D.G. Jatinder, C.H. Johnny, A new heuristic algorithm for the one-dimensional binpacking problem, Production Planning \& Control, vol. 10, no. 6, (1999) 598-603.

[46] A. Scholl, R. Klein, C. Jurgens, BISON: A fast hybrid procedure for exactly solving the one-dimensional bin-packing problem, Computers \& Operations Research, vol. 24, no. 7, (1997) 627-645.

[47] K. Fleszar, K.S. Hindi, New heuristics for one-dimensional bin-packing, Computers \& Operations Research, vol. 29, no. 7, (2002) 821-839.

[48] C.F.A. Alvim, C.C. Ribiero, F. Glover, J. D. Aloise, A hybrid improvement heuristic for the one-dimensional bin packing problem, Journal of Heuristics, vol. 10, no. 2, (2004) 205-229.

[49] K. Loh, Weight annealing heuristics for solving bin packing and other combinatorial optimization problems: concepts, algorithms and computational Results, Doctoral Thesis, University of Maryland at College Park, 2006.

[50] W. J. Dally, B. Towles, Route packets,not wires: On-chip interconnection networks, Proc. of Design Automation Conferance, (2001) 684-689.

[51] L.M. Ni, P.K. McKinley, A survey of wormhole routing techniques in direct networks, IEEE Computer (1993) 62-76.

[52] F. Moraes, N. Calazans, A. Mello, L. M'oller, L. Ost, HERMES: An infrastructure for low area overhead packet-switching networks on chip, IEEE VLSI Integration, (2004) 69-93

[53] M. Majer, C. Bobda, A. Ahmadinia, J. Teich, Packet routing in dynamically changing networks on chip, Proc. of the 19th IEEE Int. Parallel and Distributed Processing Symposium, (2005) 154-161.

[54] T. Pionteck, C. Albrecht, R. Koch, A dynamically reconfigurable packet-switched network-on-chip, Proc. of the Design Automation \& Test in Europe Conference, (2006) $1-4$.

[55] S. Corbetta, V. Rana, M.D. Santambrogio, D. Sciuto, A light-weight network-on-chip architecture for dynamically reconfigurable systems, Prof. of Int. Conference on Embedded Computer Systems: Architectures, Modeling and Simulation, (2008) 49-56.

[56] S. Jovanovic, C. Tanougast, C. Bobda, S. Weber, CuNoC: A dynamic scalable communication structure for dynamically reconfigurable FPGAs, Microprocessors and Microsystems, vol. 33, no. 3, (2009) 24-36.

[57] K. Faraydon, N. Anh, D. Sujit, An interconnect architecture for networking systems on chips, IEEE Micro, vol. 22, no. 5, (2002) 36-45.

[58] P.P. Pande, C. Grecu, A. Ivanov, R. Saleh, Design of a switch for network on chip applications, Proc. of the Int. Symposium on Circuits and Systems, (2003) 217-220. 
[59] P. Guerrier, A. Greiner, A generic architecture for on-chip packet-switched interconnections, Proceedings of the Conference on Design, Automation and Test in Europe, (2000) 250-256.

[60] J. Hu, R. Marculescu, Exploiting the routing flexibility for energy/performance aware mapping of regular NoC architectures, Design Automation and Test in Europe Conference and Exhibition, (2003) 688-693.

[61] J. Hu, R. Marculescu, Energy and performance aware mapping of regular NoC architectures, IEEE Transactions on Computer-Aided Design of Integrated Circuits and Systems, (2005) 551-562.

[62] T. Lei, S. Kumar, A two-step genetic algorithm for mapping task graphs to a network on chip architecture, Proceedings of the Euromicro Symposium on Digital System Design, (2003) 180-187.

[63] S. Murali, G. D. Micheli, Bandwidth-constrained mapping of cores onto NoC architectures, Design, Automation and Test in Europe Conference and Exhibition, (2004) $1530-1591$

[64] G. Ascia, V. Catania, M. Palesi, Multi-objective mapping for mesh-based NoC architectures, International Conference on Hardware/Software Codesign and System Synthesis (2004) 182-187.

[65] K. Srinivasan, K. S. Chatha, A technique for low energy mapping and routing in network-on-chip architectures, Proceedings of the International Symposium on Low Power Electronics and Design, (2005) 387-392.

[66] A. Hansson, K. Goossens, A. Radulescu, A unified approach to constrained mapping and routing on network-on-chip architectures, International Conference on Hardware/Software Codesign and System Synthesis (2005) 75-80.

[67] V. Nollet, T. Marescaux, P. Avasare, D. Verkesty, J. Y. Mignolet, Centralized RunTime Resource Management in a Network-on-Chip Containing Reconfigurable Hardware Tiles, Proceedings of IEEE Design Automation Test Europe, (2005) 234-239.

[68] C.L. Chou, R. Marculescu, User-aware dynamic task allocation in Networks-on-Chip, Proceedings of. Design and Automation Test, (2008) 1232-1237.

[69] C.L. Chou, R. Marculescu, Run-Time Task Allocation Considering User Behavior in Embedded Multiprocessor Networks-on-Chip, IEEE Transaction on Computer Aided Design of Integrated Circuits and Systems, (2010) 78-91.

[70] C. Steiger, H. Walder, M. Platzner, Operating systems for reconfigurable embedded platforms: online scheduling of real-time tasks, IEEE Transactions on Computers, vol. 53, no. 11, (2004) 1393-1407.

[71] W. Qiu, B. Zhoul, Y. Chen, C. Peng, Fast on-line real-time scheduling algorithm for reconfigurable computing, Proc. of the 9th Int. Conference on Computer Supported Cooperative Work in Design Proceedings, (2005) 793-798.

[72] G. Buttazzo, Hard real-time computing systems: predictable scheduling algorithms and applications, Real-Time Systems Series, vol. 23, Springer, (2005). 
[73] Xilinx ISE 12.4, http://wwW.xilinx.com/support/documentation/dt_ ise12-4.htm, last visited on August 2011.

[74] LogiCORE IP XPS HWICAP, DS586 (v 5.00a), http://wWw . xilinx.com/support/ documentation/ip_documentation/xps_hwicap.pdf, last visited on August 2011.

[75] Virtex-6 FPGA Memory Interface Solutions Data Sheet, DS186 (v 1.4), http://www . xilinx.com/support/documentation/ip_documentation/ds186.pdf, last visited on August 2011.

[76] LogiCORE IP Virtex-6 FPGA Integrated Block v1.5 for PCI Express, DS715 (v 4), http://www.xilinx.com/support/documentation/ip_documentation/v6_ pcie_ds715.pdf, last visited on August 2011.

[77] P. Schumacher, K. Denolf, A. Chilira-RUs, R. Turney, N. Fedele, K. Vissers, J. Bormans, A scalable multi-stream MPEG-4 video decoder for conferencing and surveillance applications, Proc. of IEEE Int. Conference on Image Processing, (2005) 886-889.

[78] K. Denolf, A. Chirila-Rus, D. Verkest, Low-power MPEG-4 video encoder design, Proc. of the IEEE Workshop on Signal Processing Systems Design and Implementation, (2005) 284-289.

[79] W. Hu, C. Wang, J.L. Ma, T.Z. Chen, D. Chen, A novel approach for finding candidate locations for online FPGA placement, IEEE 10th International Conference on Computer and Information Technology (2010) 2509-2515 


\section{APPENDIX A}

\section{COMPUTATIONAL STUDY RESULTS}

As was discussed in Chapter 9, an extensive computational study has been carried out to show how powerful our proposed technique is. Among 8 different task size ratio (TSR), which is the ratio of the size of the largest hardware task to the size of the smallest hardware task, only one of them has been reported in the main text and the rest is reported here. A detailed description of the computational study is given in Chapter 9.

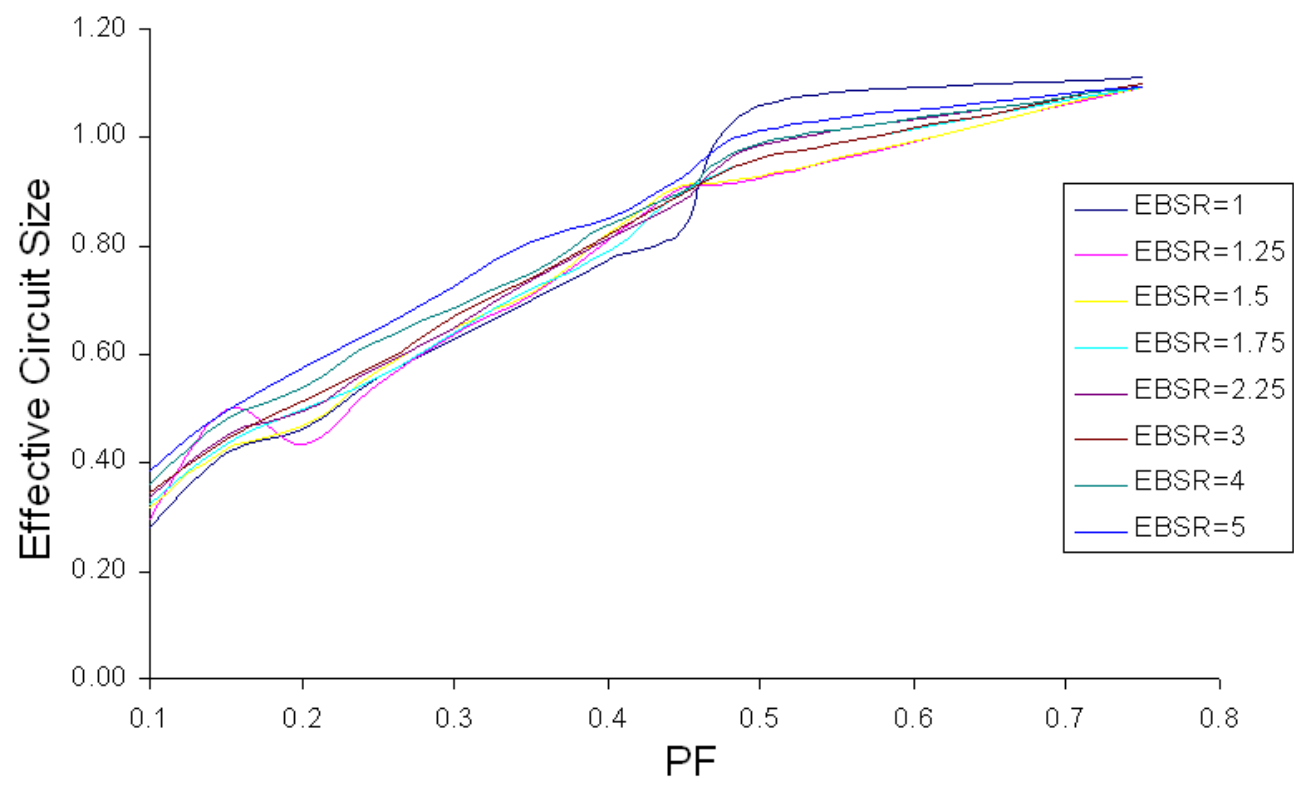

Figure A.1: Ratio of effective_size() to whole circuit size with respect to $P F$ for $T S R=1$ case 


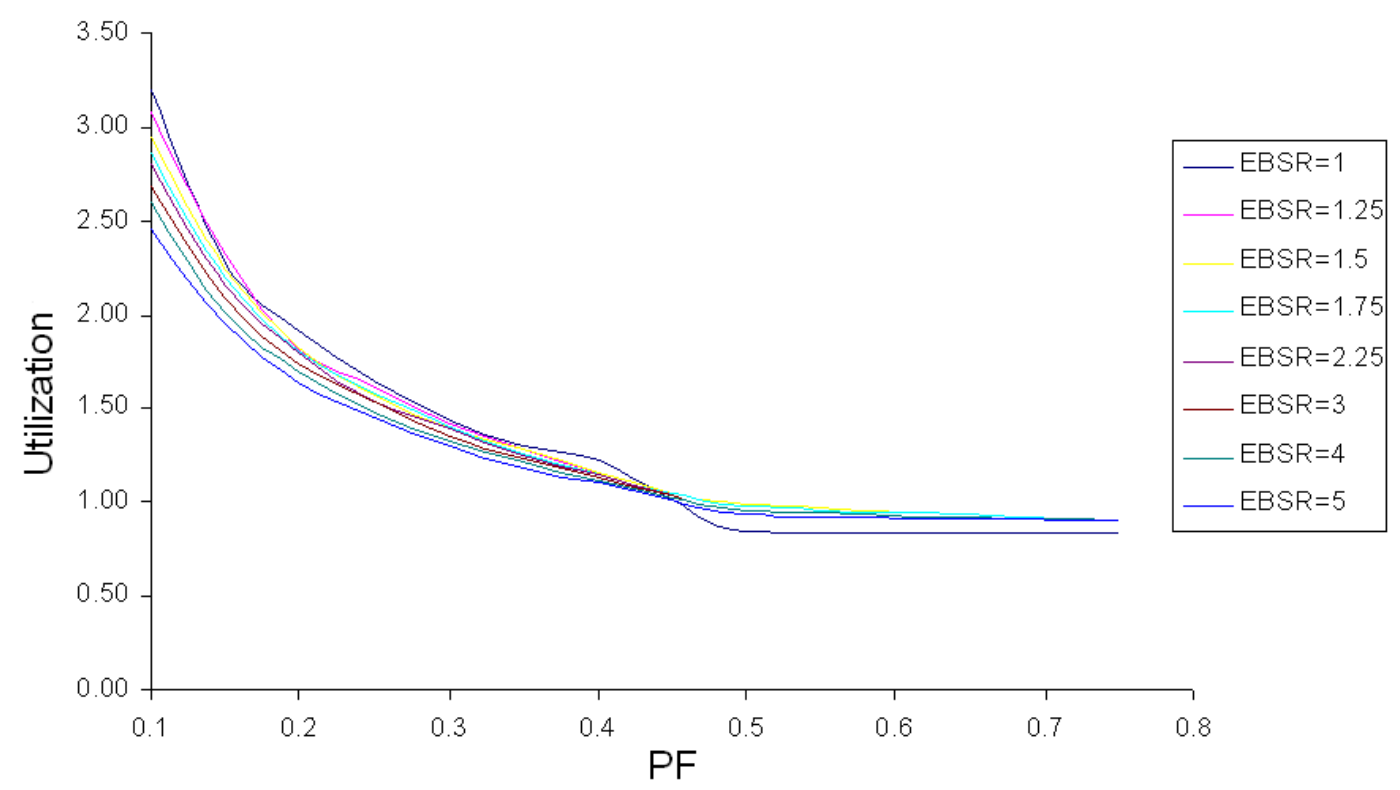

Figure A.2: $\rho_{\text {total }}$ with respect to $P F$ for $T S R=1$ case

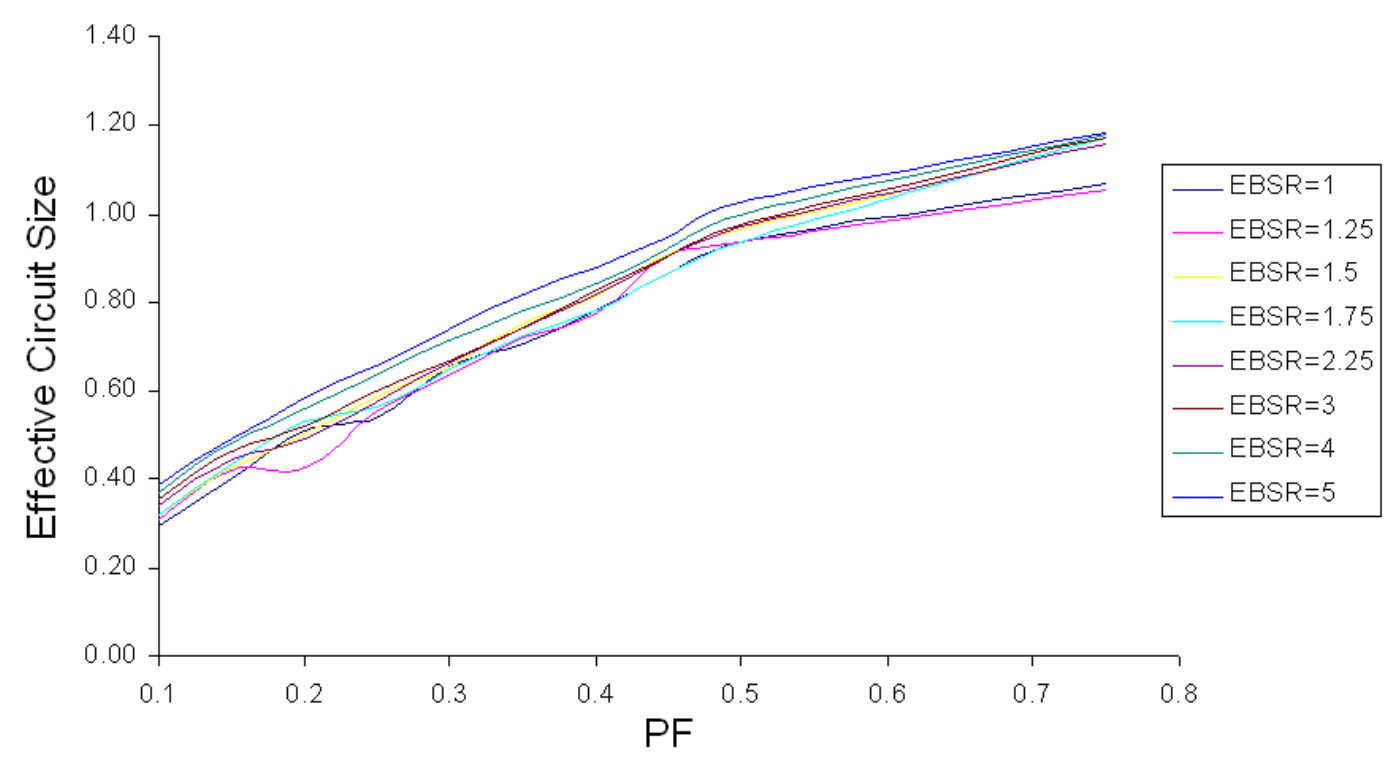

Figure A.3: Ratio of effective_size() to whole circuit size with respect to $P F$ for $T S R=1.25$ case 


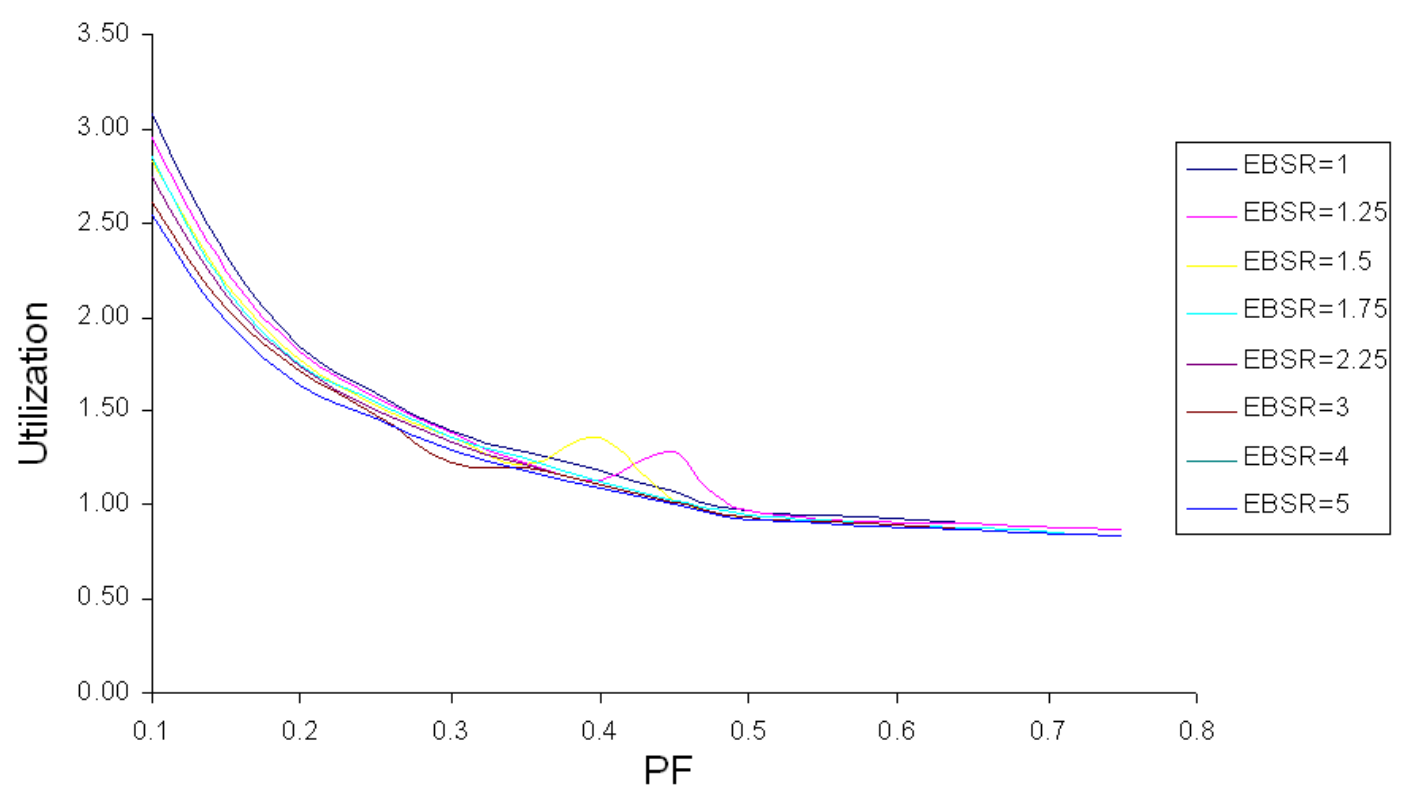

Figure A.4: $\rho_{\text {total }}$ with respect to $P F$ for $T S R=1.25$ case

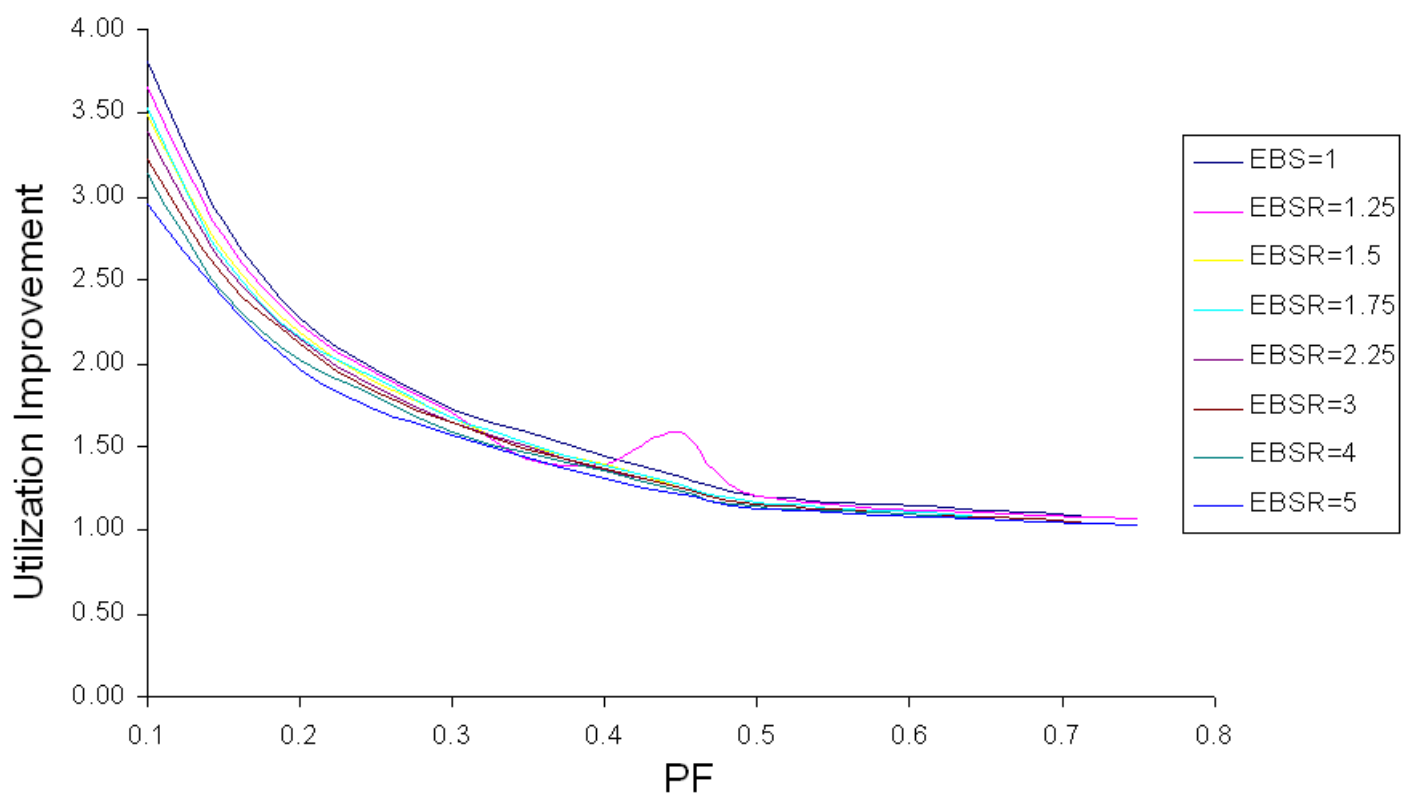

Figure A.5: Ratio of $\rho_{\text {total }}$ with our proposal to that of 2D fixed partitioning [20,21] against $P F$ for $T S R=1.25$ 


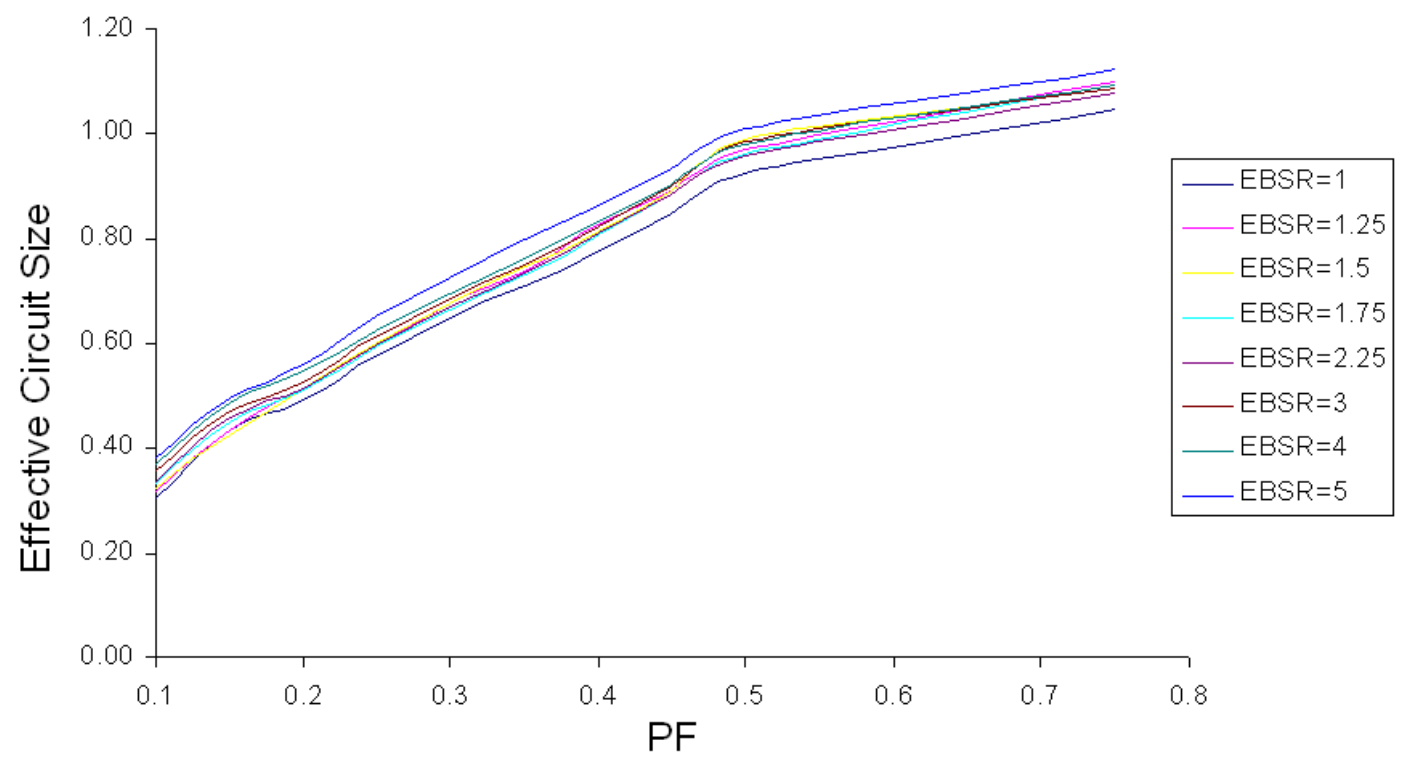

Figure A.6: Ratio of effective_size() to whole circuit size with respect to $P F$ for TSR $=1.5$ case

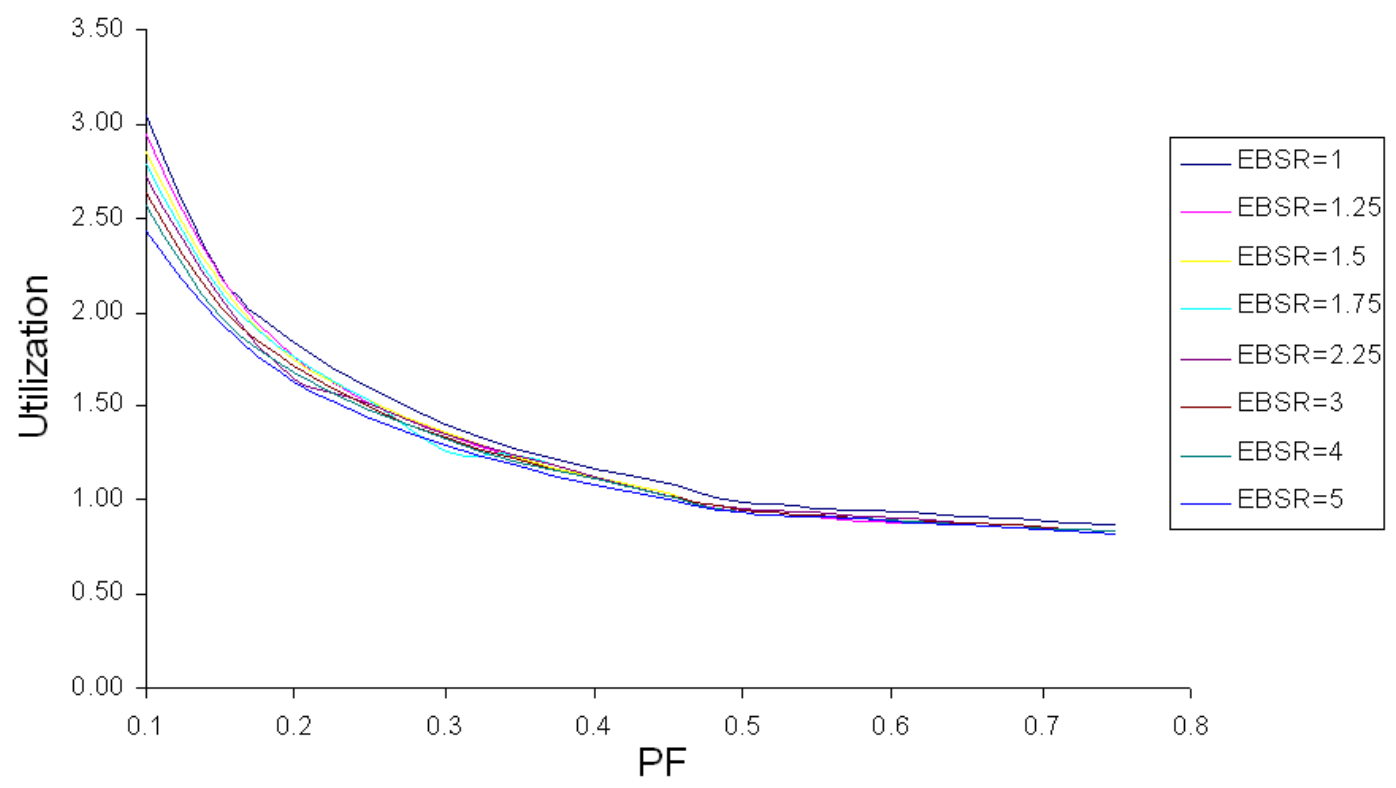

Figure A.7: $\rho_{\text {total }}$ with respect to $P F$ for $T S R=1.5$ case 


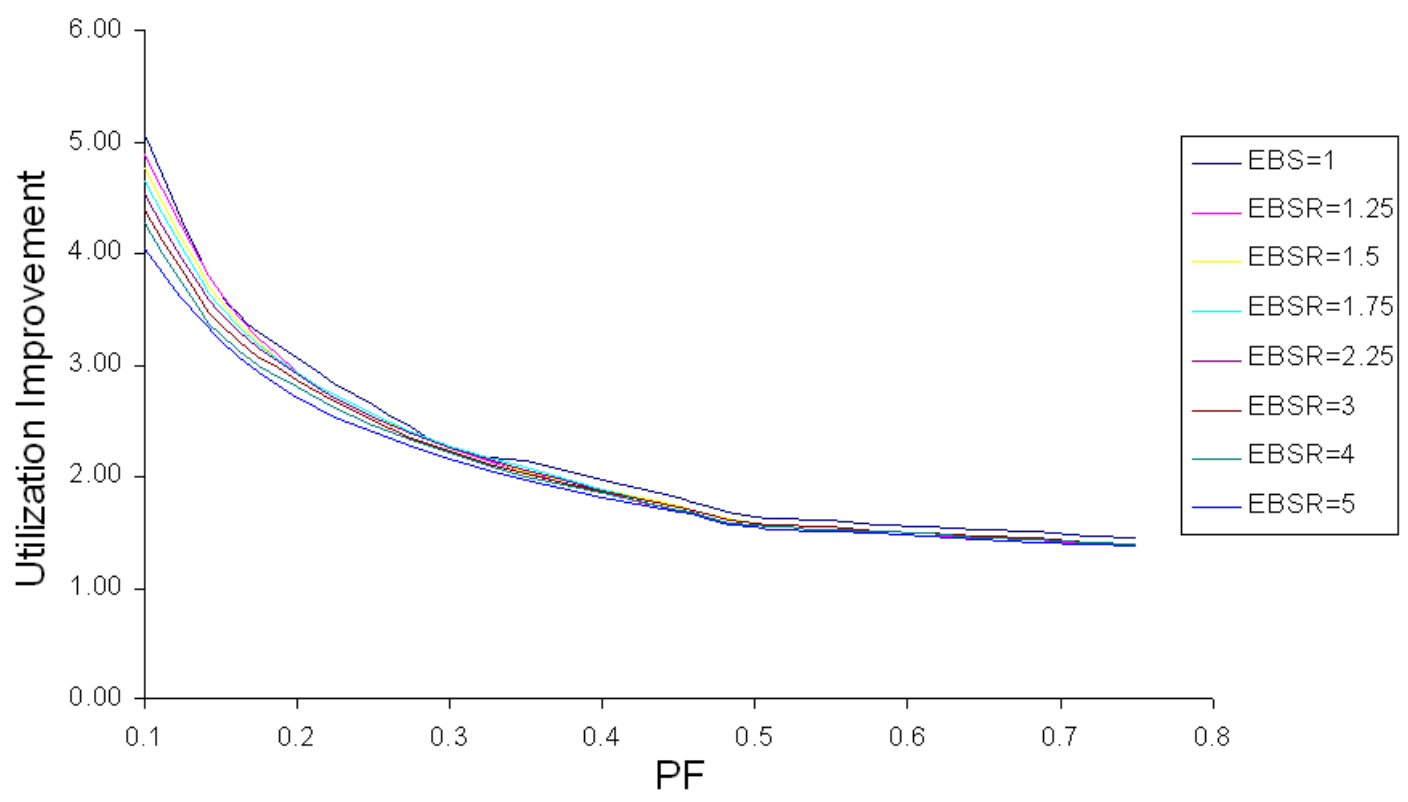

Figure A.8: Ratio of $\rho_{\text {total }}$ with our proposal to that of 2D fixed partitioning [20,21] against $P F$ for $T S R=1.5$

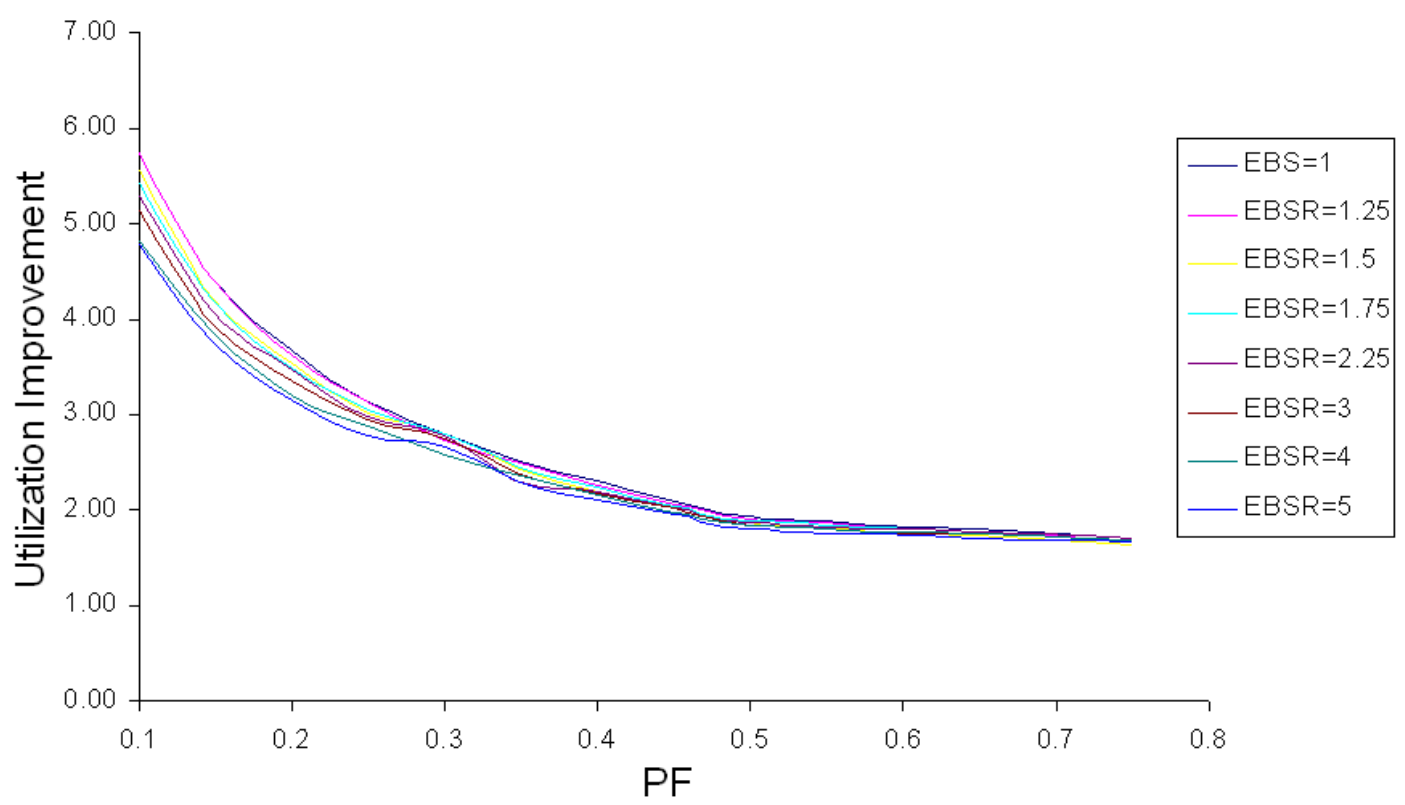

Figure A.9: Ratio of $\rho_{\text {total }}$ with our proposal to that of 2D fixed partitioning [20,21] against $P F$ for $T S R=1.75$ 


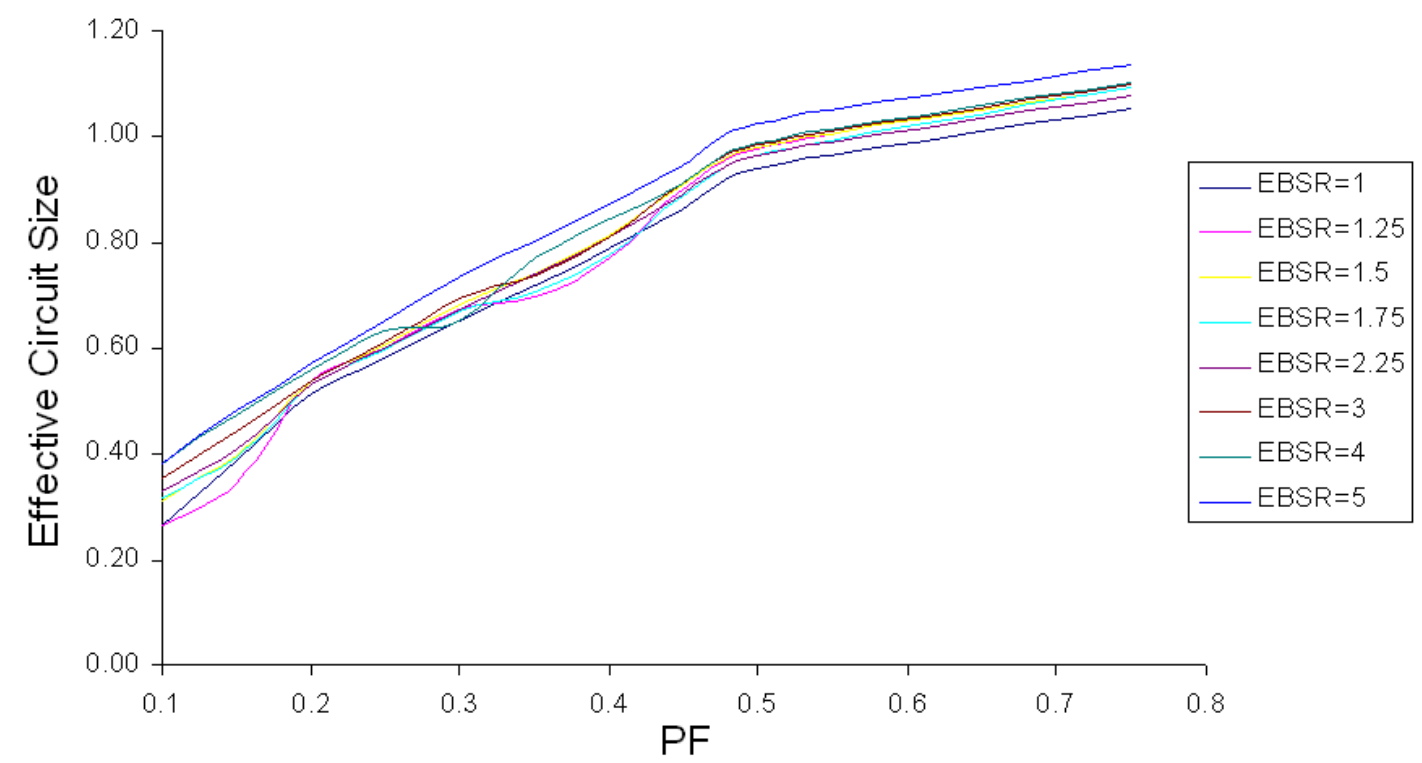

Figure A.10: Ratio of effective_size() to whole circuit size with respect to $P F$ for $T S R=$ 2.25 case

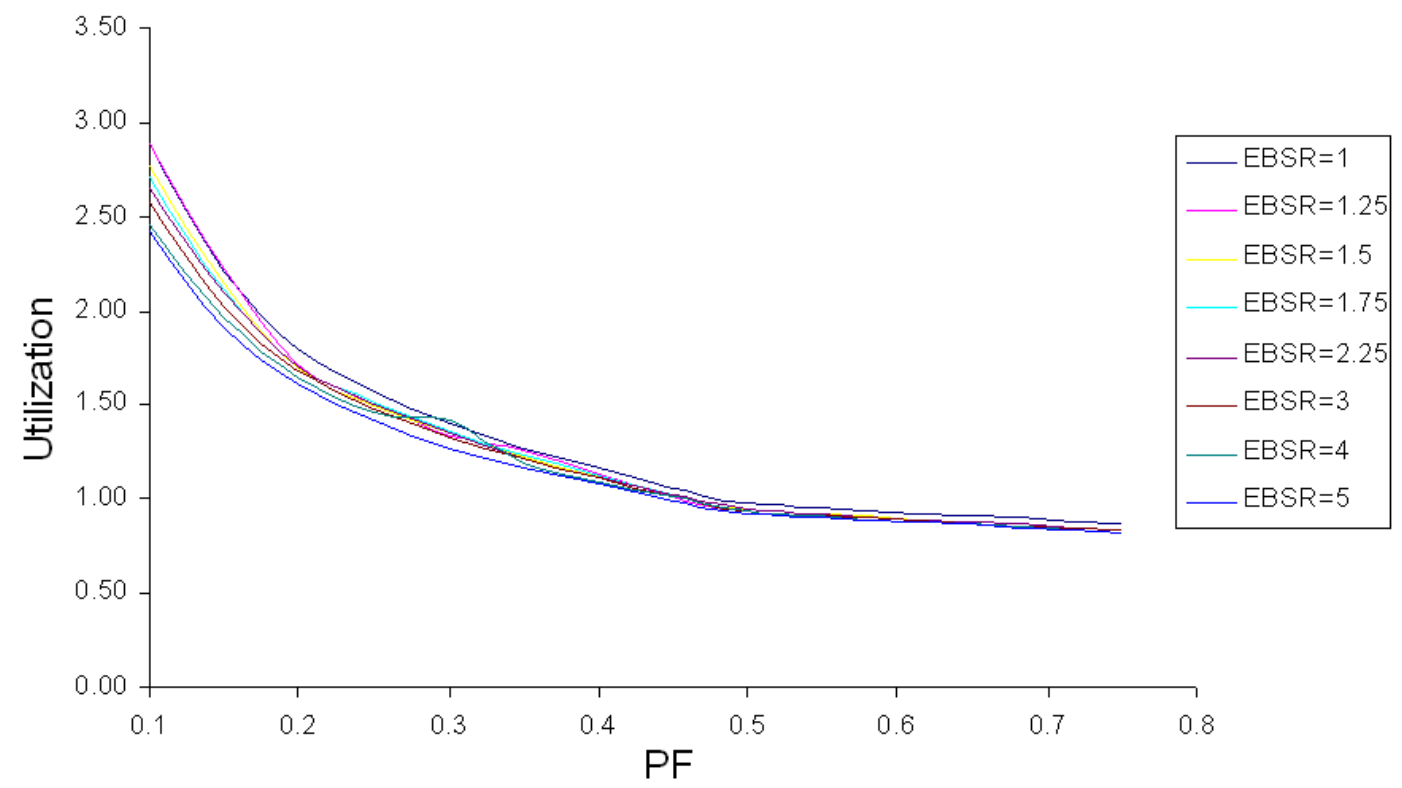

Figure A.11: $\rho_{\text {total }}$ with respect to $P F$ for $T S R=2.25$ case 


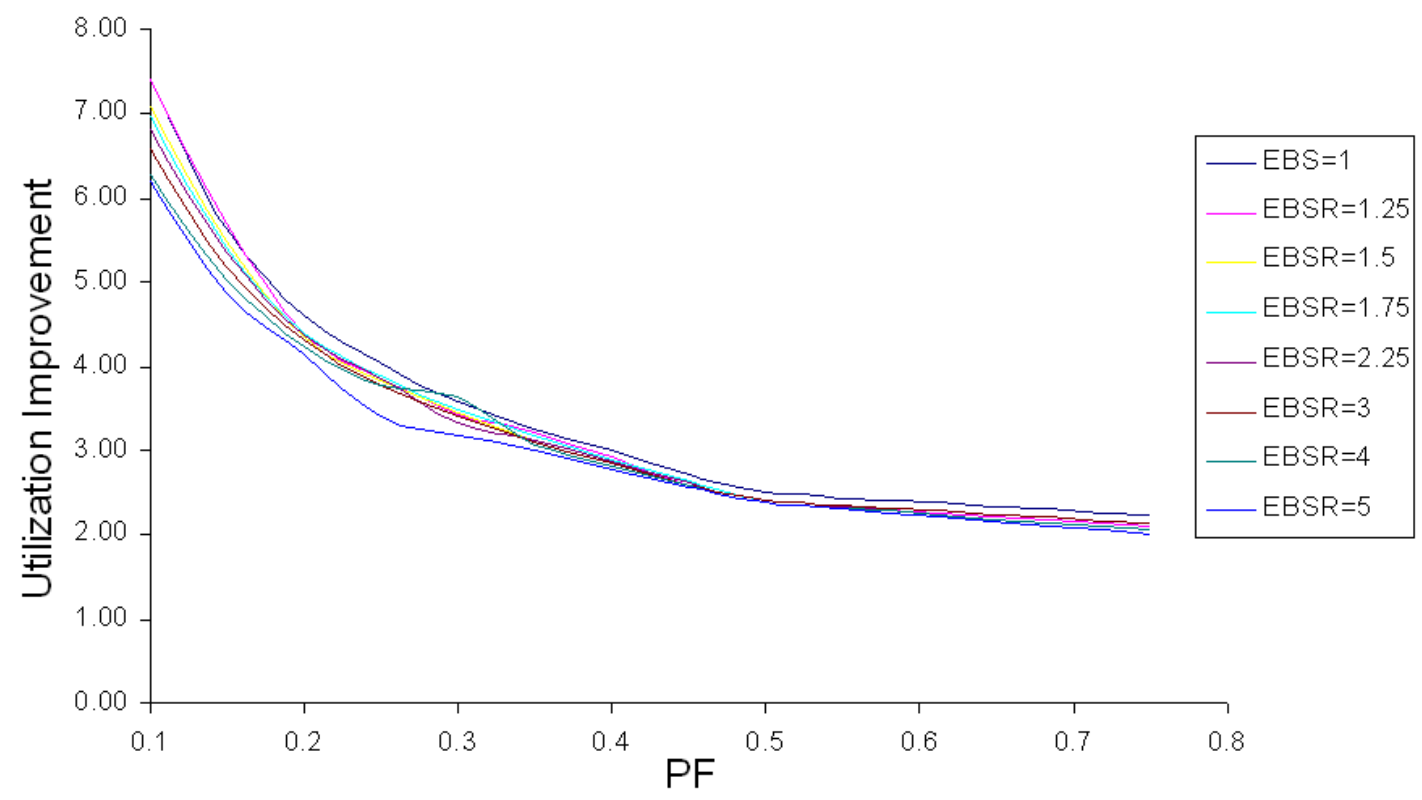

Figure A.12: Ratio of $\rho_{\text {total }}$ with our proposal to that of 2D fixed partitioning [20,21] against $P F$ for $T S R=2.25$

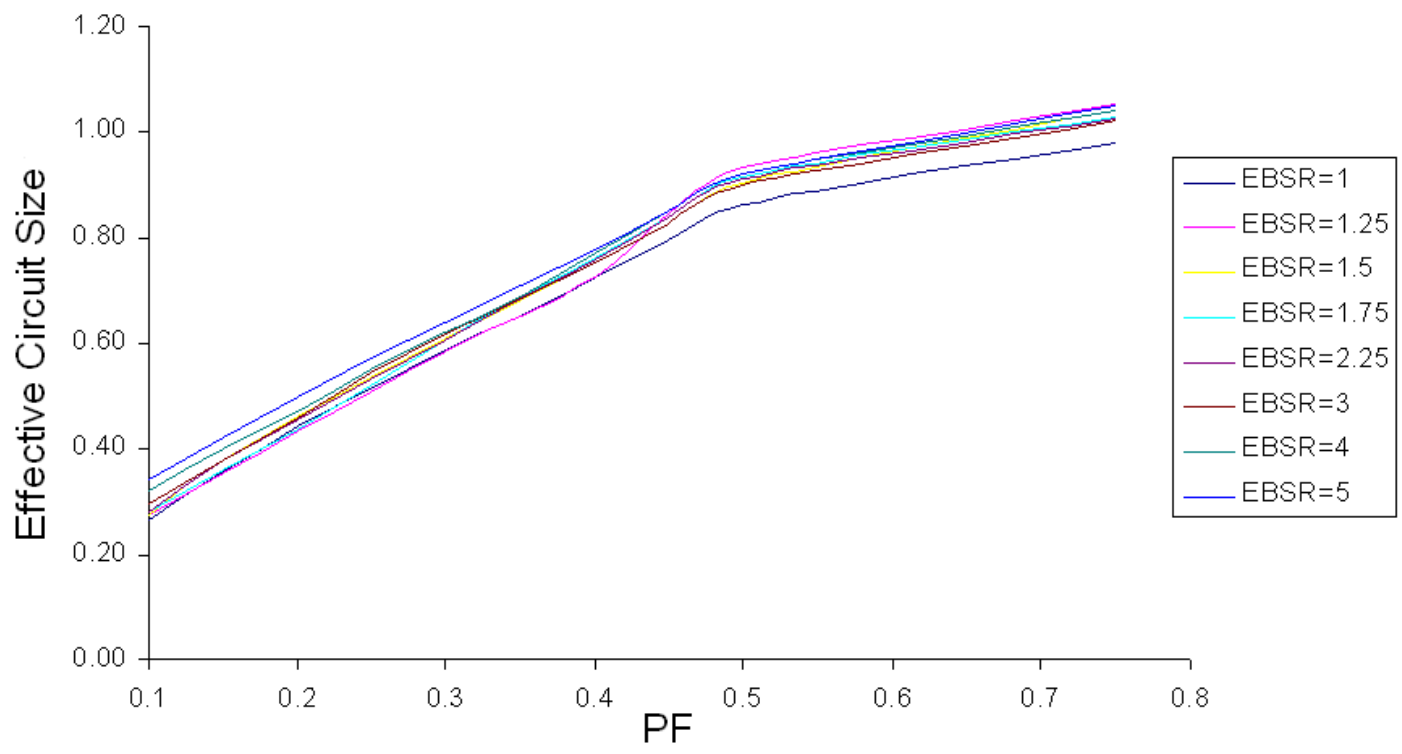

Figure A.13: Ratio of effective_size() to whole circuit size with respect to $P F$ for $T S R=3$ case 


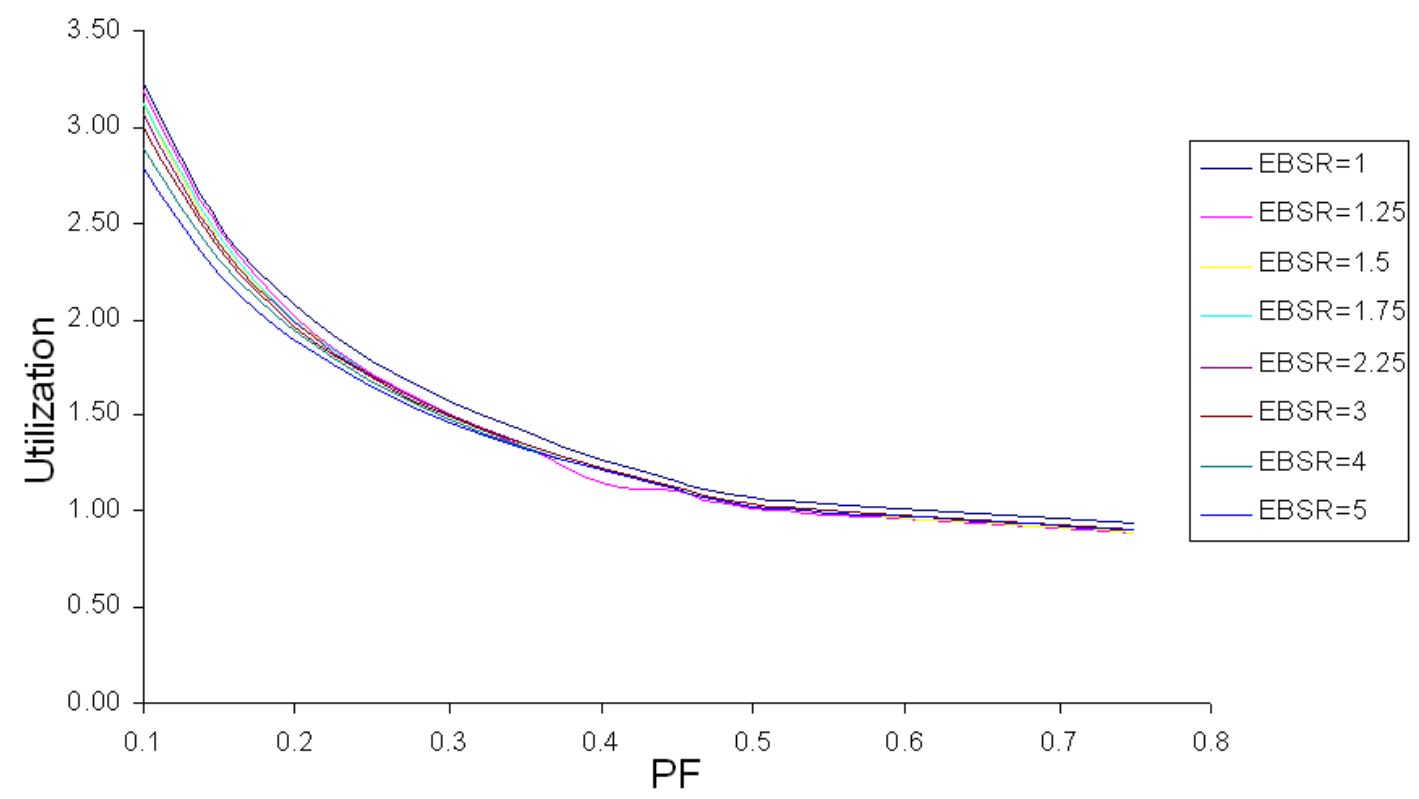

Figure A.14: $\rho_{\text {total }}$ with respect to $P F$ for $T S R=3$ case

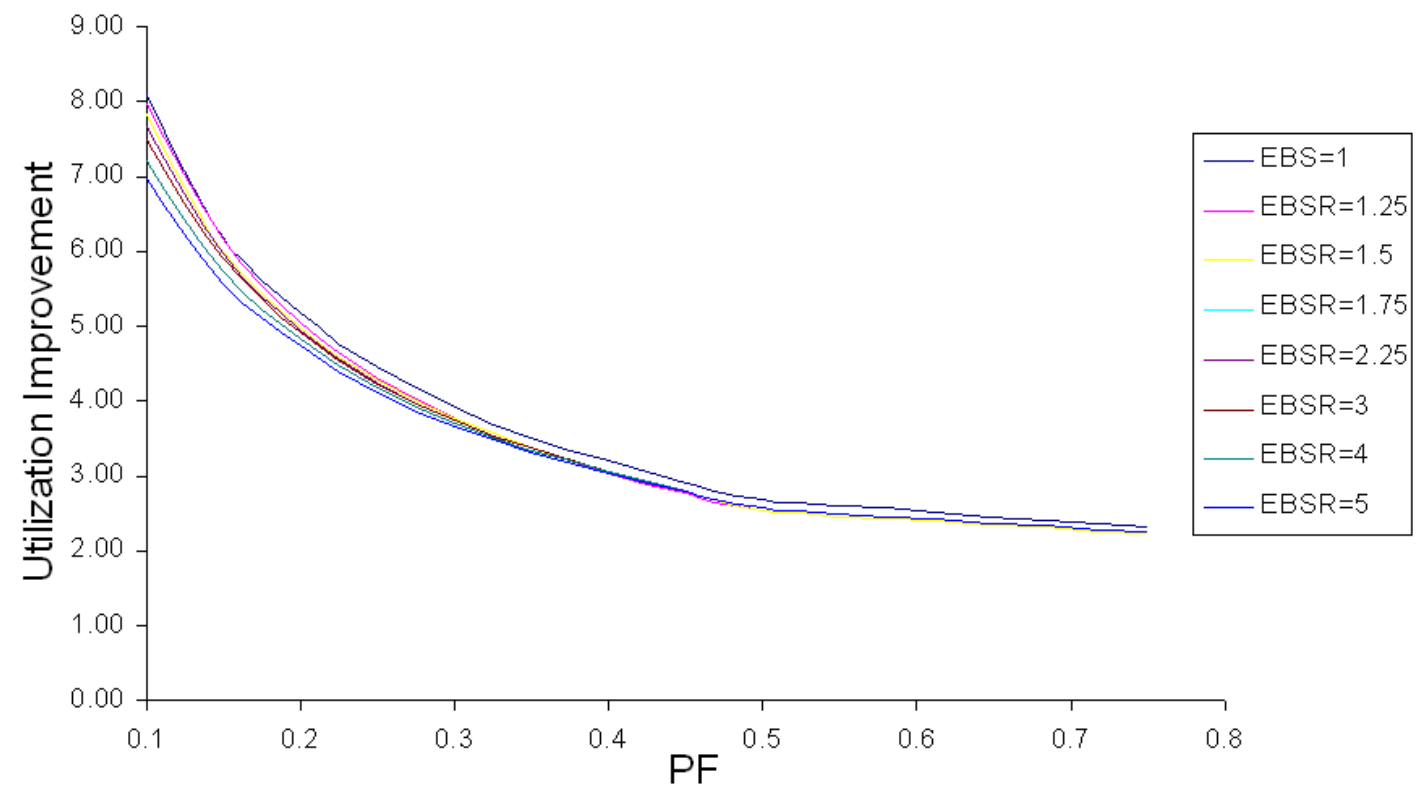

Figure A.15: Ratio of $\rho_{\text {total }}$ with our proposal to that of 2D fixed partitioning $[20,21]$ against $P F$ for $T S R=3$ 


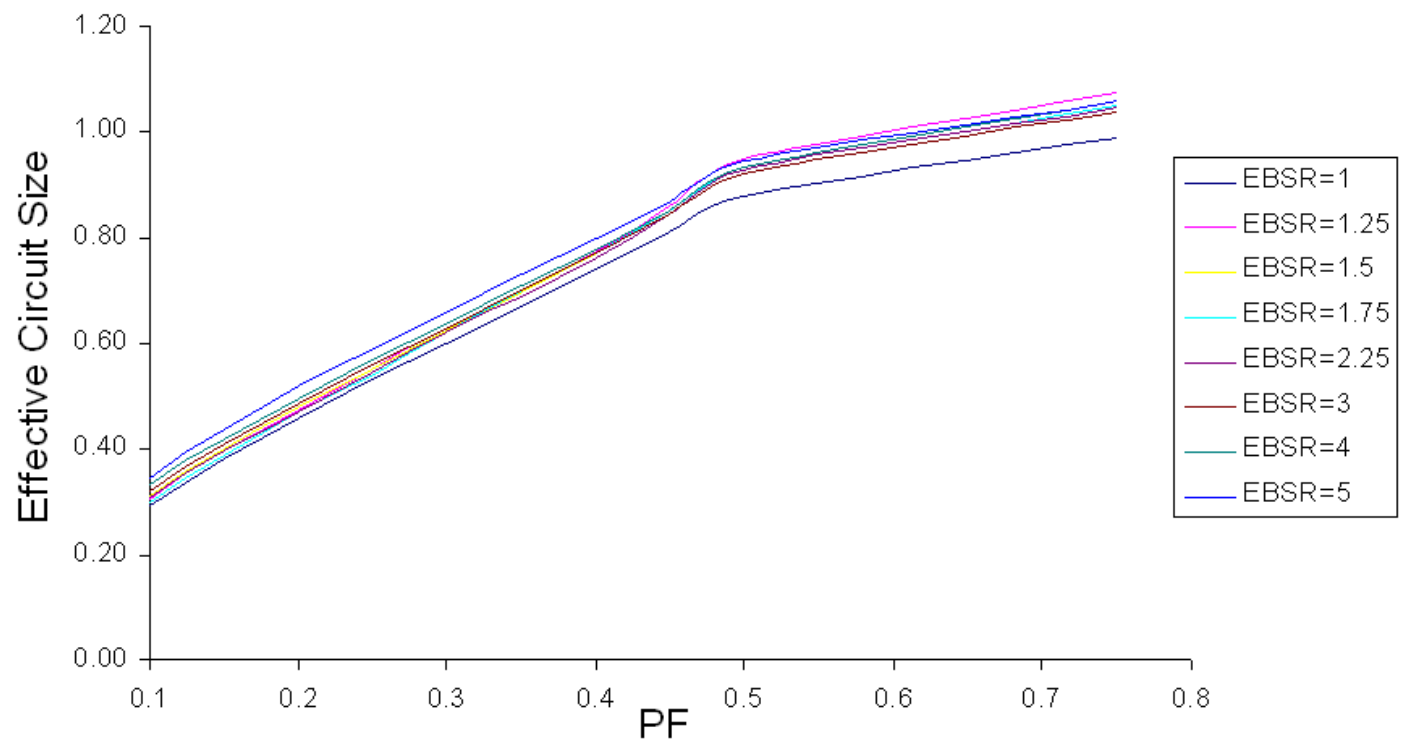

Figure A.16: Ratio of effective_size() to whole circuit size with respect to $P F$ for $T S R=4$ case

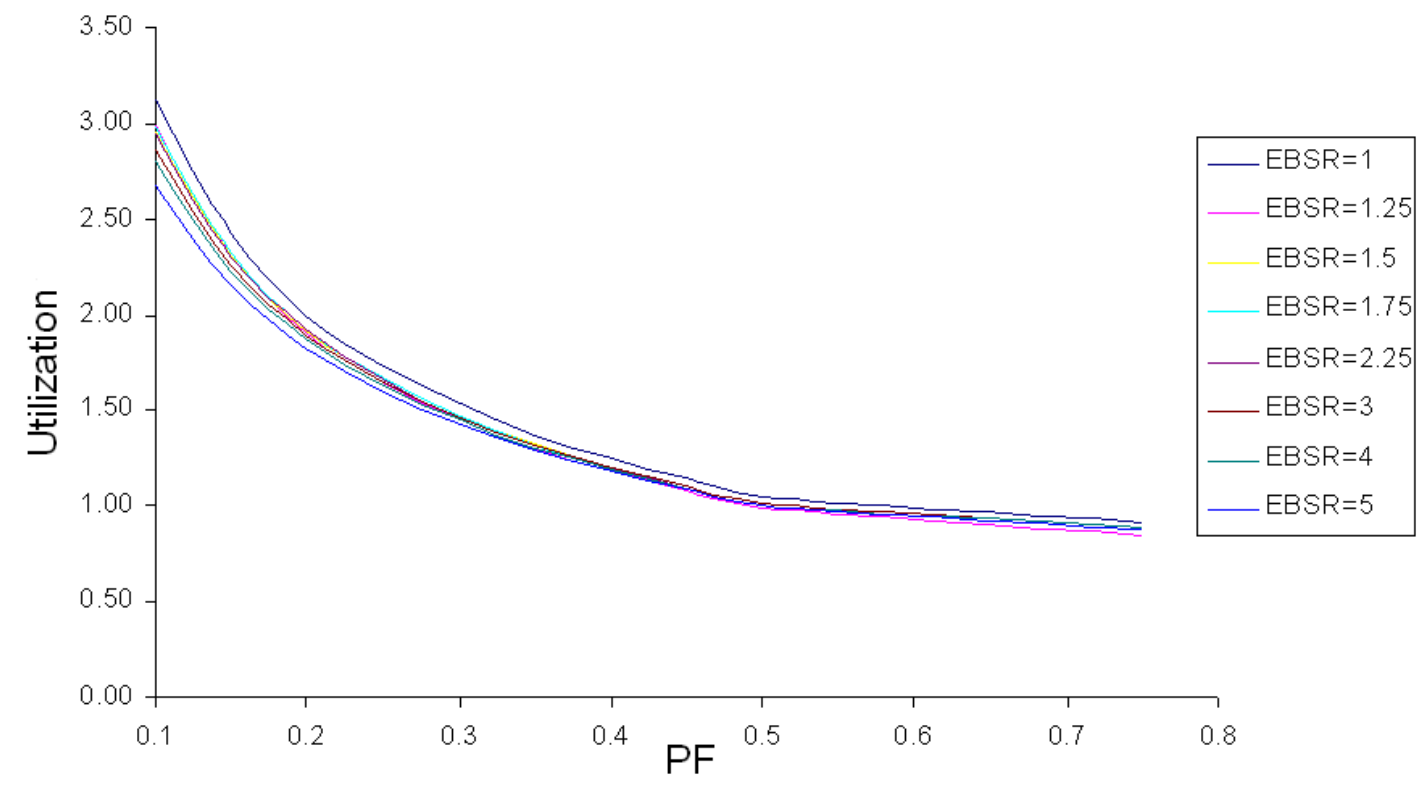

Figure A.17: $\rho_{\text {total }}$ with respect to $P F$ for $T S R=4$ case 


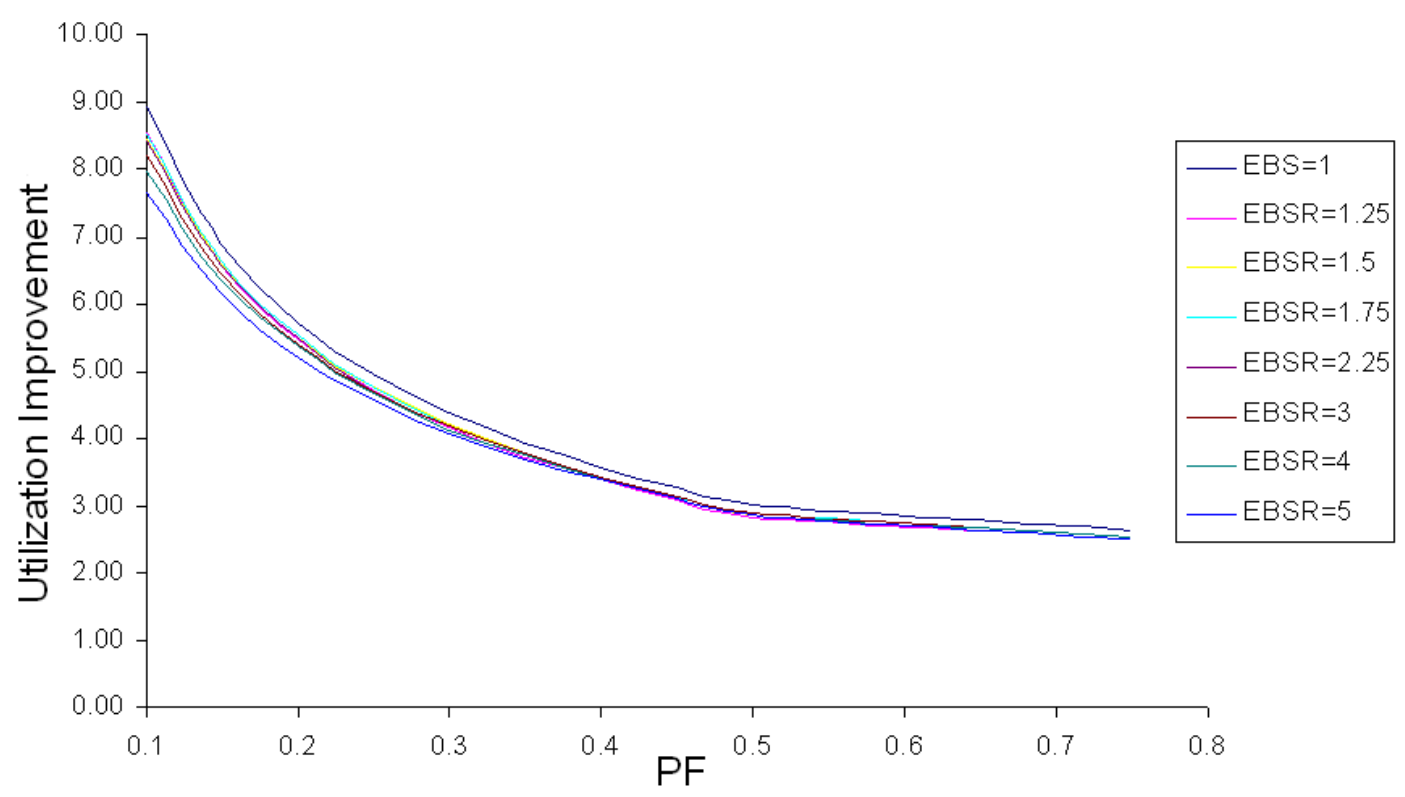

Figure A.18: Ratio of $\rho_{\text {total }}$ with our proposal to that of 2D fixed partitioning [20,21] against $P F$ for $T S R=4$

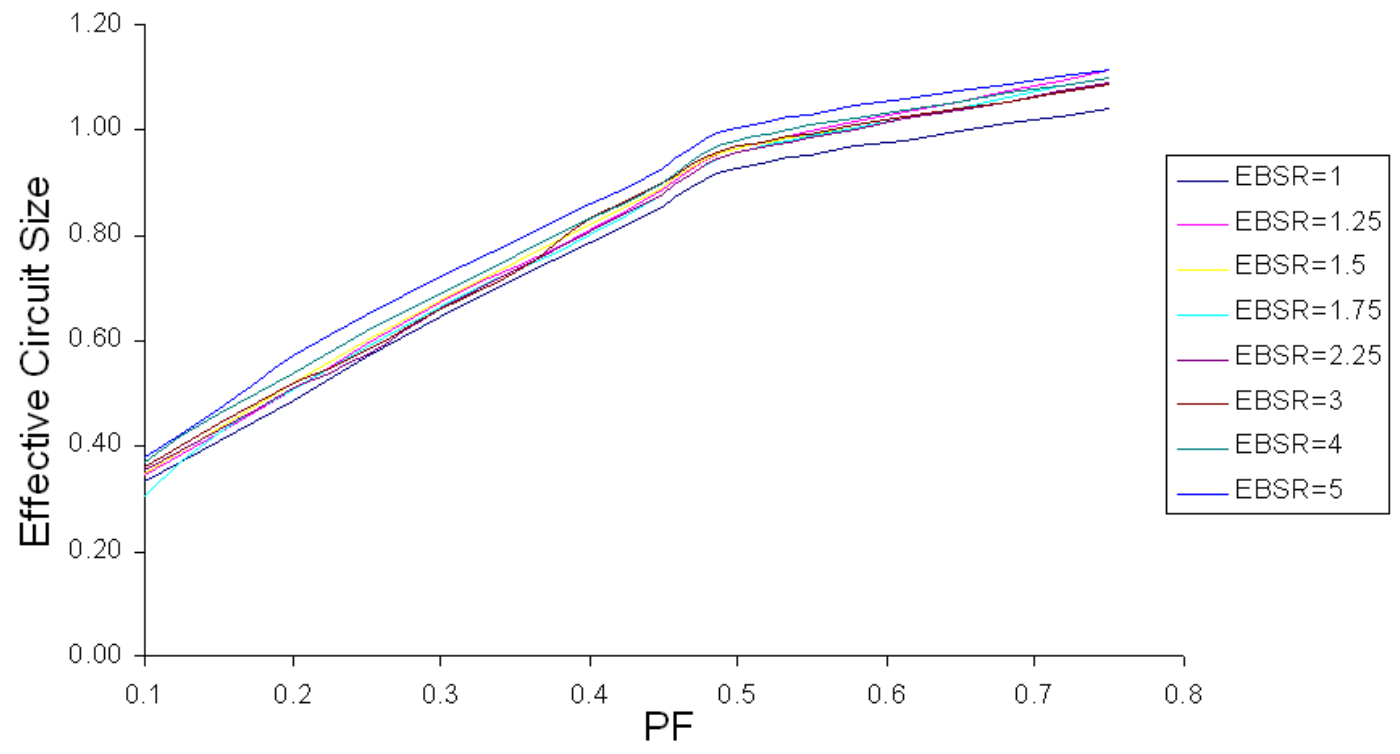

Figure A.19: Ratio of effective_size() to whole circuit size with respect to $P F$ for $T S R=5$ case 


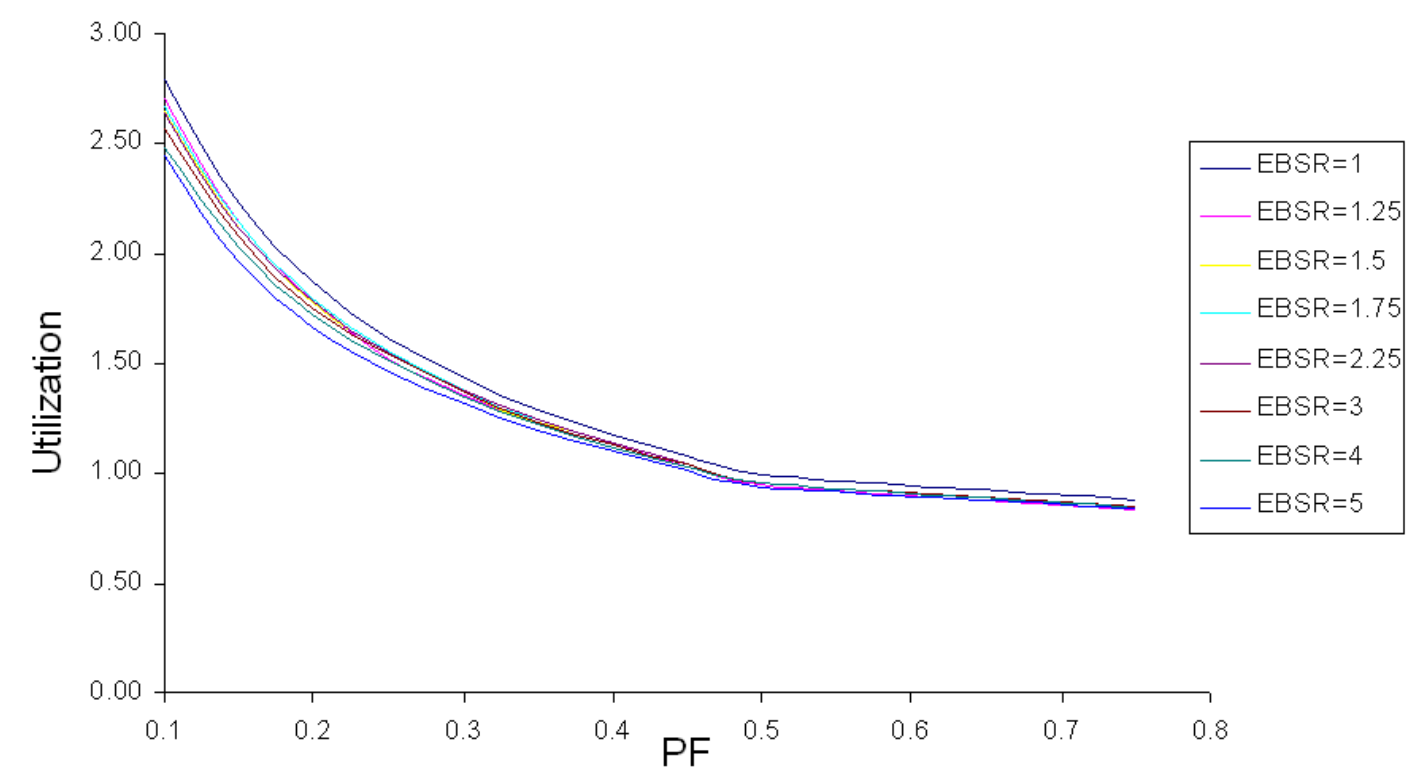

Figure A.20: $\rho_{\text {total }}$ with respect to $P F$ for $T S R=5$ case

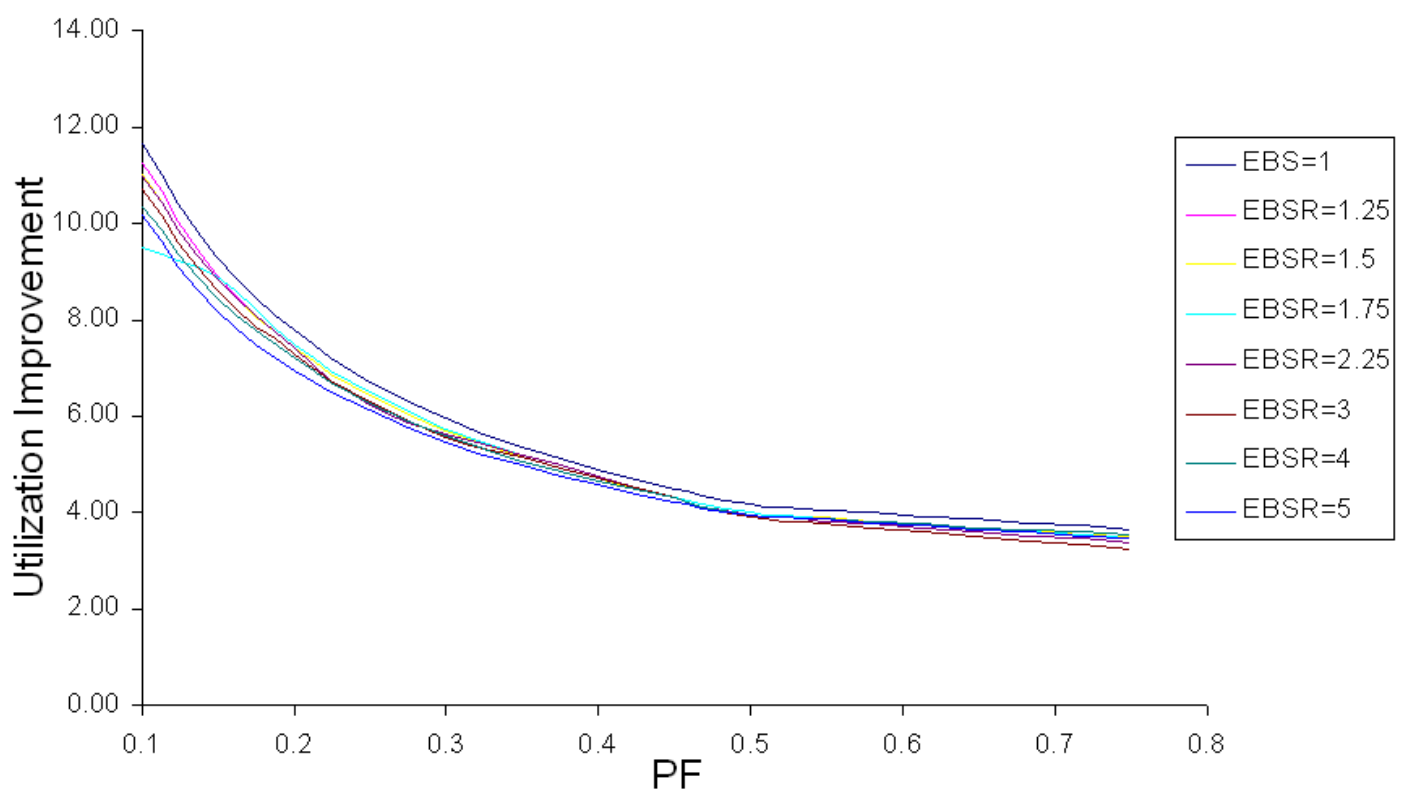

Figure A.21: Ratio of $\rho_{\text {total }}$ with our proposal to that of 2D fixed partitioning [20,21] against $P F$ for $T S R=5$ 


\title{
CURRICULUM VITAE
}

\section{PERSONAL INFORMATION}

\author{
Surname, Name $\quad$ : Say, Fatih \\ Nationality : Turkish (TC) \\ Date and Place of Birth : 19 February 1980, Van \\ Marital Status : Married \\ Phone : : +903125922549 \\ email_ : fsay@aselsan.com.tr, fatihsay@yahoo.com
}

\section{EDUCATION}

$\begin{array}{lll}\text { Degree } & \text { Institution } & \text { Year of Graduation } \\ \text { MSc } & : \text { METU Electrical and Electronics Engineering, } & 2005 \\ \text { BS } & : \text { METU Electrical and Electronics Engineering, } & 2002 \\ \text { High School } & : \text { Kazım Karabekir High School, Van } & 1997\end{array}$

\section{WORK EXPERIENCE}

$\begin{array}{lll}\text { Year } & \text { Place } & \text { Enrollment } \\ \text { 2002 December- Present } & : \text { ASELSAN A.Ş. } & \text { Senior Expert Engineer } \\ \text { 2002 June } & : \text { METU, MEMS\&VLSI Team } & \text { Research Engineer }\end{array}$

FOREIGN LANGUAGES

Advanced English

\section{PUBLICATIONS}

1. Say, F. and C.F. Bazlamaçc1, "A reconfigurable computing platform for real time embedded applications", Microprocessors and Microsystems, (2011), in press.

2. Bazlamaçcı, C.F. and Say, F. "Minimum concave cost multicommodity network design", Telecommunication Systems, (2007) 181-203 